\title{
Neutrino mass problem: the state of the art
}

\author{
Yu V Kozlov, V P Martem'yanov, K N Mukhin
}

\section{Contents}

1. Introduction

2. The current status of neutrino physics

2.1 Overview; 2.2 The history of the main discoveries in neutrino physics and in the theory of weak and electroweak interactions. The Standard Model

3. The neutrino mass problem

3.1 General considerations; 3.2 Estimates of the $v_{\mathrm{e}}$ mass from the analysis of the beta spectrum of tritium and observations of the Supernova 1987A burst; 3.3 Estimates of the masses, spins, and helicities of the muon neutrino $v_{\mu}$ and the tau neutrino $v_{\tau}$

4. Double beta decay

$4.12 \beta 2 v$ - and $2 \beta 0 v$-decay schemes. Their relation to theory and the purpose of the search; 4.2 Studying double beta decay with the xenon TPC detector; 4.3 Studying the double beta decay of ${ }^{100} \mathrm{Mo}$ and ${ }^{116} \mathrm{Cd}$ with the NEMO detector; 4.4 Studying double beta decay with a germanium detector enriched in ${ }^{76} \mathrm{Ge} ; 4.5$ Summary of the results of $2 \beta$-decay research

5. Neutrino oscillations

5.1 Neutrino mixing and oscillation hypothesis; 5.2 Mathematical formalism of neutrino oscillations; 5.3 General scheme of processing experimental data; 5.4 Search for solar neutrino oscillations. The deficit in $v_{\mathrm{e}}$ and the Mikheev-Smirnov - Wolfenstein effect. The hypothesis of the existence of a neutrino magnetic moment; 5.5 Search for atmospheric neutrino oscillations. The atmospheric neutrino anomaly; 5.6 Search for accelerator neutrino oscillations in experiments with long and short baselines; 5.7 Search for reactor antineutrino oscillations: historical survey and current experiments; 5.8 Comparison of the results of the search for oscillations of $v_{\mathrm{e}}, \tilde{v}_{\mathrm{e}}, v_{\mu}$, and $\tilde{v}_{\mu}$ in different experiments

6. New experimental projects and methods

6.1 Very large detectors of $\mu$ and $v$ with targets of unlimited size; 6.2 Superkamiokande; 6.3 The underground Sudbury Neutrino Observatory; 6.4 Other detectors for solar and atmospheric neutrinos; $6.5 \mathrm{New}$ neutrino experiments involving accelerators; $6.6 \mathrm{New}$ reactor experiments; $6.7 \mathrm{~A}$ tracer-neutrino beam; 6.8 Artificial radioactive isotopes as sources of neutrinos

7. Possible practical uses of neutrinos

7.1 Neutrino diagnostics of nuclear reactors. Estimates of reactor power and composition of core; 7.2 Neutrinos in geophysics. Neutrino geology and geodesy

8. Conclusions

References

\begin{abstract}
Current knowledge of the neutrino mass, double $\beta$ decay, and neutrino oscillations is reviewed and recent neutrino experiments are discussed in detail. The discovery and basic studies of $v_{\mathrm{e}}, v_{\mu}$, and $v_{\tau}$ are briefly described in historical perspective. Projected experiments and techniques as well as prospects for the practical use of the neutrino are covered.
\end{abstract}

Yu V Kozlov, V P Martem'yanov, K N Mukhin Russian Research Centre 'Kurchatov Institute', Institute of General and Nuclear Physics

pl. Kurchatova 1, 123182 Moscow, Russia

Tel. (7-095) 196-7729

E-mail:kozlov@chen.net.kiae.su,vpm@vpmar.kiae.su, mukhin@chen.net.kiae.su

Received 18 December 1996, revised 30 April 1997 Uspekhi Fizicheskikh Nauk 167 (8) 849 - 885 (1997)

Translated by E Yankovsky; edited by A Radzig

\section{Introduction}

Early in December 1930, the young and famous by that time theoretical physicist Wolfgang Pauli sent a letter to the participants of a physics meeting held in Tübingen. He declared that he was unable to be present at the gathering because he wanted to attend the famous annual ball at the Swiss Federal Institute of Technology. The letter began 'Dear Radioactive Ladies and Gentlemen'. Despite such an unusual salutation and the general free style of the letter [1,2], it is the most remarkable scientific paper in nuclear physics. In this letter Pauli suggested the existence of a new, spin-1/2, light, uncharged, and highly penetrating particle, later called the neutrino (Pauli called it the neutron). This neutrino hypothesis made it possible to solve two very important problems in nuclear physics of the time: the incorrect statistics of the nitrogen nucleus (the 'nitrogen catastrophe') and the continuous spectrum of electrons in beta decay. 
In 1930, this was a revolutionary step, and few people took the idea seriously. And Pauli himself, being believed in it, thought that because of the very small cross section of the interaction between a neutrino and matter experimenters would never be able to prove its existence. He even betted on this, but luckily lost.

Twenty-three years have passed. In $1953-1956$ a series of experiments conducted by Reines and Cowan (deceased 1974) [3] proved the existence of the neutrino. According to modern nomenclature, the particle discovered should be called an electron neutrino $\left(v_{\mathrm{e}}\right) \dagger$. The triplet of neutrinos (since then the muon neutrino $\left(v_{\mu}\right)$ and the tau neutrino $\left(v_{\tau}\right)$ have also been discovered) occupies the centre stage of elementary particle physics:. Judging by the modern status of neutrino physics and the number of highly difficult problems that have yet to be solved, a strong interest in these particles will stand up for years and in any event it will cross the threshold of the next century.

The present article is an attempt to express the rather difficult question of the modern status of neutrino physics and especially the problem of neutrino mass using fairly simple language.

The plan is as follows. In Section 2 we give an overview of the development of neutrino physics within the framework of the physics of weak and electroweak interactions. Section 3 is devoted to the problem of neutrino mass. There we give the experimental and cosmological estimates of the masses of neutrinos of different types $\left(v_{\mathrm{e}}, v_{\mu}, v_{\tau}\right)$ and a detailed description of the modern experiments, as well as we discuss the existence possibilities of $m_{v}=0$ and $m_{v} \neq 0$ and the interrelationship between $m_{v} \neq 0$, the $2 \beta 0 v$-decay, and neutrino oscillations. Section 4 deals with neutrinoless and twoneutrino $2 \beta$-decays, their features, the experimental methods employed, and the results achieved. In Section 5 we cover neutrino oscillations: the conditions needed for their existence, the mathematical formalism, and the parameters utilized. We give the story of the search for neutrino oscillations in solar, atmospheric, accelerator, and reactor neutrinos. New experimental projects and methods are examined in Section 6, and possible practical applications involving neutrinos are considered in Section 7. Finally, Section 8 is a summary of the material discussed.

In writing this review we have used material from books, papers, preprints, and reports of conferences up to 1996. Among the reviews close to the topics of the various sections in this paper we point out those by Zatsepin and Smirnov [5] and Muto and Klapdor [6] (the general properties of neutrinos), Gelmini and Roulet [7] and Boehm and Vogel [8] (neutrino masses), Wojcicki [9] (neutrino oscillations and the masses of $v_{\mu}$ and $v_{\tau}$ ), Moe and Vogel [10,11] and Shchepkin [12] (double beta decay), Borovor and Khakimov [13] (experiments with reactor neutrinos), Weinheimer et al. [14] and Belesev et al. [15] (the mass of $v_{\mathrm{e}}$ ), and Imshennik and Nadezhin [16]

\footnotetext{
$\uparrow$ Strictly speaking, the particle discovered was not $v_{\mathrm{e}}$ but the antiparticle $\tilde{v}_{\mathrm{e}}$; however, according to the principle of charge conjugation, each particle has an antiparticle. For proving the existence of a free neutrino in a direct experiment, examining the process $\tilde{v}_{\mathrm{e}}+\mathrm{p} \rightarrow \mathrm{n}+\mathrm{e}^{+}$, Frederick Reines awarded the 1995 Nobel Prize for physics [4]. (Nobel prizes are not given posthumously.)

\$ Actually the existence of $v_{\tau}$ has not been proved in experiments of the Reines-Cowan type, but it follows irrevocably from tau-lepton decay and the theory of weak interactions.
}

(Supernova 1987A). The values of some quantities are those accepted as of April 1997.

The review, written by experimenters, is intended for a broad range of physicists (not only specialists in neutrino physics). Therefore we do not discuss highly specialized theoretical problems, but sometimes mention them so that the reader is aware of the problems.

\section{The current status of neutrino physics}

\subsection{Overview}

For the time being let us ignore the subtle aspects (neutrino mass, $2 \beta 0 v$-decays, and oscillations). If we limit ourselves to the firmly established facts, the following picture of the current status of neutrino physics emerges§.

In addition to three electrically charged leptons with halfinteger spins, the electron $\mathrm{e}^{-}$, the muon $\mu^{-}$, and the heavy lepton $\tau^{-}$, whose masses are roughly $0.5,106$, and $1784 \mathrm{MeV}$, respectively, three neutral leptons occur also naturally with half-integer spins whose masses are most probably very close to (or maybe even exactly equal to) zero. These three uncharged leptons are three different neutrinos: the electron neutrino $\left(v_{\mathrm{e}}\right)$, the muon neutrino $\left(v_{\mu}\right)$, and the tau neutrino $\left(v_{\tau}\right)$, the names signifying that in the process of their interaction with matter (nucleons) they usually come together with the corresponding charged leptons $\$$.

Phenomenologically, the difference between neutrino types (flavours) as long as the difference between the charged leptons $\mathrm{e}^{-}, \mu^{-}$, and $\tau^{-}$are described by introducing three independent lepton numbers $L_{i}=+1(i=\mathrm{e}, \mu, \tau)$ and the respective conservation laws. All the antileptons $\left(\mathrm{e}^{+}, \mu^{+}, \tau^{+}\right.$, $\left.\tilde{v}_{\mathrm{e}}, \tilde{v}_{\mu}, \tilde{v}_{\tau}\right)$ are associated with $L_{i}=-1$, while hadrons, with $L_{i}=0$.

According to the laws of conservation of lepton numbers (with allowance made for antiparticles), the following processes, for instance, are possible:

$$
\begin{aligned}
& \mathrm{n} \rightarrow \mathrm{p}+\mathrm{e}^{-}+\tilde{v}_{\mathrm{e}}, \quad \tilde{v}_{\mathrm{e}}+\mathrm{p} \rightarrow \mathrm{n}+\mathrm{e}^{+}, \\
& v_{\mu}+\mathrm{n} \rightarrow \mu^{-}+\mathrm{p}, \quad \tilde{v}_{\mu}+\mathrm{p} \rightarrow \mu^{+}+\mathrm{n},
\end{aligned}
$$

while the following processes are forbidden:

$$
\begin{aligned}
& v_{\mathrm{e}}+\mathrm{p} \nrightarrow \mathrm{n}+\mathrm{e}^{+}, \quad v_{\mathrm{e}}+\mathrm{n} \nrightarrow \mu^{-}+\mathrm{p}, \\
& \tilde{v}_{\mathrm{e}}+\mathrm{p} \nrightarrow \mu^{+}+\mathrm{n} .
\end{aligned}
$$

The validity of the reactions (1) and (2) (and many other reactions, which either satisfy or do not satisfy the laws of conservation of lepton numbers) has been corroborated in numerous experiments; for instance, a proof that $\tilde{v}_{\mathrm{e}}$ exists was obtained from the reaction $\tilde{v}_{\mathrm{e}}+\mathrm{p} \rightarrow \mathrm{n}+\mathrm{e}^{+}$[3], while the difference between $\tilde{v}_{\mathrm{e}}$ and $v_{\mathrm{e}}$ was verified by the absence of the reaction $\tilde{v}_{\mathrm{e}}+\mathrm{n} \rightarrow \mathrm{p}+\mathrm{e}^{-}$[22] (the difference between $v_{\mu}$ and $\tilde{v}_{\mu}$ was checked in a similar manner).

$\S$ A more thorough review of the general status of neutrino physics and the physics of weak interactions can be found in the book by $\mathrm{Wu}$ and Mozkowski [2] and (up to 1980) in the review by Pontecorvo [17]. An elementary review of modern aspects of experimental neutrino physics has been made by Mukhin [18], while a more theoretical approach was taken by Okun' [19]. Finally, Rekalo's book [20] covers a detailed history of neutrino physics and the role that various scientists played in forming the idea of the neutrino (with numerous quotations from the works of these scientists). The masses and other parameters of elementary particles are given according to the 1996 'Review of Particle Properties' [21].

- The number (three) of different types of neutrino follows from the experimentally determined width of the $Z^{0}$-boson (see Section 2.3). 
The difference between $\tilde{v}_{\mathrm{e}}$ and $v_{\mathrm{e}}, v_{\mu}$ and $v_{\mathrm{e}}, v_{\tau}$ and $v_{\mathrm{e}}, v_{\mu}$ and $v_{\tau}$ can be estimated by the overlap of the corresponding wave functions, $\left|\Psi_{i} \Psi_{j}\right|^{2}$ (which must be zero if the particles are absolutely different). Experiments have shown that $\left|\Psi_{v_{\mathrm{e}}} \Psi_{v_{\mu}}\right|^{2} \approx 0.001$ and $\left|\Psi_{v_{\mathrm{e}}} \Psi_{v_{\tau}}\right|^{2} \approx\left|\Psi_{v_{\mu}} \Psi_{v_{\tau}}\right|^{2} \approx 0.01$ [5]. Estimates of the difference between $v_{\mathrm{e}}$ and $\tilde{v}_{\mathrm{e}}$ are given below in Section 3.1.

All types of neutrinos (and antineutrinos) participate only in weak and gravitational interaction with ordinary matter (nucleons and electrons), since they have neither electric charge, nor colour charge, and nor detectable magnetic moment (see Section 5.4.5). The cross section of the interaction between neutrinos and the matter (nucleons) at $E_{v} \approx 1 \mathrm{MeV}$ is approximately $10^{-43} \mathrm{~cm}^{2}$ and increases with energy first quadratically and then linearly $\left[\sigma_{v}(1 \mathrm{GeV}) \approx\right.$ $\left.\approx 0.7 \times 10^{-38} \mathrm{~cm}^{2}\right]$. It is precisely because this interaction cross section is so small that the existence of an electron antineutrino $\left(\tilde{v}_{\mathrm{e}}\right)$ was proved experimentally only in $1953-$ 1956 , i.e. approximately a quarter of a century after its remarkable theoretical prediction.

The gathering of experimental data on the properties of neutrinos was parallel to the development of the universal theory of the four-fermion weak interaction, which forms the basis of the modern theory of the electroweak interaction (the Standard Model) and yields correct results in the low-energy range $\left(E \ll m_{\mathrm{W}}\right.$, where $m_{\mathrm{W}} \approx 90 \mathrm{GeV}$ is the mass of the intermediate vector $\mathrm{W}$-boson).

\subsection{The history of the main discoveries in neutrino physics and in the theory of weak and electroweak interactions. The Standard Model}

The first (vector) variant of the weak interaction theory was set up in 1934 by Enrico Fermi [23], who proposed the idea of the point (contact) interaction of four fermions, $\mathrm{n}, \mathrm{p}, \mathrm{e}^{-}$, and $v$, to explain the beta decay of nuclei.

Fermi's theory played a very important role in the development of weak interaction physics. It explained the two types of beta decay (the only types known at that time, $\beta^{-}$- and $\beta^{+}$-decay), described the electron and positron spectra, classified the beta radioactive nuclei according to their decay probabilities (allowed and forbidden transitions), and estimated the weak coupling constant $G$. Fermi's theory likewise predicted new phenomena that had yet to be discovered (electron capture, inverse beta decay, scattering of $v_{\mathrm{e}}$ by e, and weak nuclear forces), and also played an important part in neutrino physics. Actually, the theory gave the direction for the search for neutrinos in the indirect experiments of Leipunsky [24] in 1936 and Allen [25] in 1942 and in the direct experiments of Reines and Cowan [3] in $1953-1956$. However, the purely vector variant of the theory was unable to explain the beta decay of some nuclei, e.g. ${ }^{6} \mathrm{He}$, such a process possessing a high probability, as was shown experimentally. Therefore, the theory was modified, and in addition to the vector variant (V) an axial-vector (A) variant appeared in the $(\mathrm{V}-\mathrm{A})$ form.

The following theoretical and experimental discoveries played an important role in the development of the $(\mathrm{V}-\mathrm{A})$ theory. In 1956, Lee and Yang [26] theoretically, and in 1957, $\mathrm{Wu}$ et al. [27] and Lederman with collaborators [28] experimentally, proved the violation of the law of spatial parity $(\mathrm{P})$ conservation and the breakdown of charge $(\mathrm{C}$ invariance) in weak interactions, as well as the left-handed polarization (of order $v / c$ ) for leptons and right-handed polarization (of order $-v / c$ ) for antileptons. Direct measure- ments of the polarization of the electron neutrino were carried out by Goldhaber and coworkers [29] in 1958. Slightly earlier in 1957, Landau [30], Salam [31], and Lee and Yang [32] proposed a two-component theory of the neutrino, according to which the neutrino and antineutrino have opposite helicities and zero masses.

Finally, in 1957-1958, combining the existing experimental facts and theoretical ideas, Feynman and Gell-Mann [33], on the one hand, and independently Sudarshan and Marshak [34] and Sakurai [35], on the other, were able to put together a universal four-fermion weak interaction theory. The main propositions of this theory are as follows:

(1) the universal and contact nature of the four-fermion interaction;

(2) the $(\mathrm{V}-\mathrm{A})$ variant of the theory;

(3) the conservation of the weak vector current;

(4) $100 \%$ violation of $\mathrm{P}$ - and $\mathrm{C}$-invariance;

(5) the left-handed polarization of all leptons and the right-handed polarization of all antileptons;

(6) $100 \%$ conservation of lepton numbers, $\tilde{v} \neq v$;

(7) the agreement between the theoretical results and the assumption that $m_{v} \equiv 0$.

The universal theory of the weak interaction has been verified and is still being verified in numerous experiments.

A lot of attention (and this attention is caught for more than 40 years!) is focused on experiments on measuring the neutron lifetime and the angular correlations between the neutron spin and the momenta of decay products, as well as experiments on determining the lifetime of $\beta$-radioactive nuclei with $\left(0^{+}-0^{+}\right)$-transitions.

The choice of such nuclei and the neutron is determined by the fact that in both cases the squares of the matrix elements of the beta transitions are known exactly, in view of which the $(\mathrm{V}-\mathrm{A})$ theory makes it possible to determine the vector coupling constant $G_{\mathrm{V}}$ for each of these entities. Here, the law of conservation of the weak vector current (Zel'dovich and Gershtein [36]) implies that the $G_{\mathrm{V}}$ for the beta decay of nuclei and for the beta decay of a neutron are the same. This requirement of the theory was fulfilled as long as the accuracy with which the $G_{\mathrm{V}}$ were measured was not high. Lately, however, a discrepancy has been observed, which could be caused (in addition to experimental error) either by an admixture of right-handed $(\mathrm{V}+\mathrm{A})$-currents within the $(\mathrm{V}$, $\mathrm{A}$ )-variant or by an admixture of other (S, T)-variants (for details see the review by Mostovoi et al. [37], the papers by Gaponov and Khafizov [38-40], and an earlier work by Gaponov [254]). Thus, the above experiments served as a good test for verifying the $(\mathrm{V}-\mathrm{A})$-theory.

We would like to list some additional important problems and discoveries that are directly related to neutrino physics and the problem of the weak interaction. In 1961, Glashow [41] proposed a gauge theory of electroweak interactions. In 1962, Lederman, Schwartz and collaborators [42] conducted an experiment (suggested by Pontecorvo [43]) to prove that $v_{\mu}$ differs from $v_{\mathrm{e}}$. In the next year they also proved [44] that $v_{\mu}$ differs from $\tilde{v}_{\mu}$.

In 1964, Gell-Mann [45] and independently Zweig [46] suggested a baryonic triplet consisting of three hypothetical particles (u, d, s), which Gell-Mann called quarks, with fractional baryonic and electric charges

$$
B_{\mathrm{u}, \mathrm{d}, \mathrm{s}}=\frac{1}{3}, \quad q_{\mathrm{u}}=+\frac{2}{3}|e|, \quad q_{\mathrm{d}}=q_{\mathrm{s}}=-\frac{1}{3}|e| .
$$


In the $(\mathrm{V}-\mathrm{A})$-theory of interaction these three quarks naturally replaced the proton, the neutron, and the lambdaparticle.

The year 1964 was also rich in experimental results. Christenson et al. [47] discovered the violation of CPinvariance in the decay of $\mathrm{K}_{L}^{0}$-mesons. This discovery inspired physicists to start a search, which still continues (see the review by Mostovor et al. [37]), for the neutron's electric dipole moment, whose existence requires that P-parity and Tinvariance be violated simultaneously, which according to the CPT-theorem is equivalent to CP-invariance violation. In the same year, Abov et al. [48] discovered P-parity violation in nuclear interactions, i.e. actually discovered the weak nucleon-nucleon forces. In 1966, a similar result was obtained by Lobashev et al. [49], who used a different method.

In 1967-1968, Weinberg [50] and Salam [51] built a unified renormalizable gauge theory of electromagnetic and weak interactions with four vector bosons: $\mathrm{W}^{+}, \mathrm{W}^{-}, \mathrm{Z}^{0}$, and $\gamma$. The Weinberg-Glashow-Salam theory predicted the existence of a fourth, charmed, quark (or c-quark with $B_{\mathrm{c}}=1 / 3$ and $\left.q_{\mathrm{c}}=(+2 / 3)|e|\right)$, neutral weak currents, the masses of the $\mathrm{W}^{ \pm}$and $\mathrm{Z}^{0}$-bosons, the value of the Weinberg angle $\Theta_{\mathrm{W}}$, and other things. In 1970, Glashow et al. [52] introduced the c-quark into the theory, and in this way symmetrized the theory with respect to the number of quarks and leptons. Then in 1973, Hasert et al. [53] used the reaction $v_{\mu}+\mathrm{N} \rightarrow v_{\mu}+$ hadrons to discover weak neutral currents, which, in contrast to weak charged currents (cf. the reaction $v_{\mu}+\mathrm{N} \rightarrow \mu^{-}+$hadrons), do not alter the electric charge of leptons. In 1974, Aubert and collaborators [54] and Augustin and collaborators [55] simultaneously and independently discovered the $J / \Psi$-particle (the bound state of c- and $\tilde{\mathrm{c}}$ quarks).

The third charged lepton, or the heavy $\tau$-lepton, was discovered in 1975-1977 by Perl and collaborators [56]. Studies of its decay resulted in the conclusion that there must be a third neutrino, the tau neutrino $v_{\tau}$. Now there were six leptons. In 1977, Lederman and collaborators (see Ref. [57]) discovered the upsilon meson (the bound state of band $\tilde{\mathrm{b}}$-quarks), which meant there were now five quarks $\left(B_{\mathrm{b}}=1 / 3\right.$ and $\left.q_{\mathrm{b}}=(-1 / 3)|e|\right)$. The symmetry in the properties of leptons and quarks requires that there be equal number of each, but the sixth, t-quark, whose parameters had been predicted long ago $\left(B_{\mathrm{t}}=1 / 3\right.$ and $\left.q_{\mathrm{t}}=(+2 / 3)|e|\right)$, was discovered only in 1996. Its mass is [21]

$$
m_{\mathrm{t}}=180 \pm 12 \mathrm{GeV} \text {. }
$$

The history of neutrino physics and electroweak interactions abounds with other interesting events. In 1983, the quanta of weak interaction, the $\mathrm{W}^{ \pm}$- and $\mathrm{Z}^{0}$-bosons, were discovered [58-60]. The masses of these particles were found to be

$$
m_{\mathrm{W}^{ \pm}}=81.0 \pm 1.3 \mathrm{GeV}, \quad m_{\mathrm{Z}^{0}}=92.4 \pm 1.8 \mathrm{GeV},
$$

which was in full agreement with the predictions of the Weinberg-Glashow-Salam theory. The years that followed brought many new experimental verifications of the validity of this theory, which became known as the Standard Model of electroweak interactions. In contrast to the universal four-fermion theory of the weak contact interaction, the electroweak interaction in the Standard Model is realized by the exchange of the vector bosons $\mathrm{W}^{+}, \mathrm{W}^{-}, \mathrm{Z}^{0}$, and $\gamma$. Other aspects of the universal theory of the weak interaction mentioned earlier, such as $\tilde{v} \neq v, m_{v} \equiv 0,100 \% \mathrm{P}$ - and $\mathrm{C}$-invariance violation but conservation of lepton numbers, left-handed polarization of neutrinos and righthanded polarization of antineutrinos, are also valid in the Standard Model.

In the 1990s several new superaccelerators became operational, and hundreds of thousands of events representing the production and decay of the $\mathrm{Z}^{0}$-boson were detected. This resulted in highly accurate measurements of the mass of this boson [21]:

$$
m_{\mathrm{Z}^{0}}=91.187 \pm 0.007 \mathrm{GeV} .
$$

The measurements of the decay parameters of $Z^{0}$ (total and partial widths) yielded the following bound on the number of light neutrinos:

$$
n_{v}=3.09 \pm 0.13 \text {. }
$$

The mass of the $\mathrm{W}^{ \pm}$-bosons was also determined more accurately:

$$
m_{\mathrm{W}^{ \pm}}=80.33 \pm 0.15 \mathrm{GeV} .
$$

The common gauge nature of strong and electroweak interactions allows us to bargain on the development of a theory that generalizes these interactions, or a Grand Unification Theory with a symmetry group [possibly, SU(5)] that is broader than the constituent groups [U(1), SU(2), and $\mathrm{SU}(3)$ ]. Since this group incorporates both quarks and leptons, transitions violating the laws of conservation of lepton and barion numbers may occur between these particles, say, proton decay. A contemporary estimate of the proton lifetime is

$$
\tau_{\mathrm{p}}>10^{31}-5 \times 10^{32} \text { years }
$$

(depending on the decay mode).

\section{The neutrino mass problem}

\subsection{General considerations}

The fundamental properties of neutrino $v_{\mathrm{e}}$, including the approximate value of its mass $\dagger$, were already obvious at the time when the theoretical predictions were made. In his famous letter [1], Pauli wrote that the neutrino mass must be of the order of the electron mass, and in any case no larger than one-hundredth of the proton mass.

Naturally, when he predicted the main parameters of the neutrino (zero electric charge, spin, high penetrating power, and a fairly small mass), Pauli had no way of specifying all the properties of this particle exactly, and in some aspect his predictions were not corroborated. In addition to the neutrino mass being too large, he believed, for instance, that the neutrino was a constituent of atomic nuclei.

The next step in establishing the properties of the neutrino was taken by Fermi (incidentally, he introduced the modern name of this particle) in his remarkable paper written in 1934, 'An attempt on the theory of $\beta$-rays' [23]. At roughly the same time (1932) the neutron was discovered, and the protonelectron model of the atomic nucleus was replaced by the proton-neutron model, according to which there could be no electrons inside a nucleus. Fermi's hypothesis asserts that

+ At the time nothing was known about the existence of three different neutrinos, $v_{\mathrm{e}}, v_{\mu}$, and $v_{\tau}$, and all the researchers 'simply' spoke about a neutrino $v$ (even calling it a neutron). However, to avoid any confusion, we will use the modern notation, $v_{\mathrm{e}}$. 
neutrino is also absent in the nucleus. Instead, the electron and the neutrino were brought about (created) at the instant of $\beta$-decay and immediately left the nucleus. Fermi also assumed that the neutrino mass is small compared to the electron mass or is even zero and proposed an experimental method for estimating the neutrino mass by the behaviour of the $\beta$-spectrum near its edge (for more details see Section 3.2).

It so happens that knowing which of the two possibilities $\left(m_{v}=0\right.$ or $\left.m_{v} \neq 0\right)$ is realized in nature is highly important. Let us discuss this aspect, basing our reasoning on the available experimental findings and current theoretical ideas.

At present, experimental data yield only upper bounds on the neutrino masses. For $m_{v_{\mathrm{e}}}$ this is roughly $\sim 5 \mathrm{eV}$ (Section 3.2.4), for $m_{v_{\mu}}$ roughly $0.17 \mathrm{MeV}$, and for $m_{v_{\tau}}$ roughly $24 \mathrm{MeV}$ (Section 3.3). Thus, experiments provide no solution to the problem. What, then, does the theory say? First we note that the theoretical notion about the neutrino mass is modeldependent, i.e. it is determined by the theoretical model of the neutrino nature. Here are the most well-known models: the Standard Model, and the theories of Dirac, Majorana, and Weyl. We begin with the Standard Model.

The reader will recall that, according to the Standard Model, $m_{v}=0$ (massless neutrino), there exist completely polarized left-handed neutrinos and right-handed antineutrinos, the weak interaction is controlled by left-handed (V-A)currents, and the lepton numbers are strictly conserved. This is the common, extremely convenient model (it is not without reason that the model was called Standard), which contradicts no experimental fact. But suppose that the neutrino mass is not exactly zero (a massive neutrino). Then this assumption leads to such important consequences (neutrino instability, a nonzero magnetic moment, oscillations, and the possibility of solving some important astrophysical problems $f$ ) that it is worthwhile stepping outside the Standard Model, the more so that the experiments verifying the theoretical results are not absolutely correct but only within a certain error and there occurs no first physical principle that requires the neutrino to be massless. The question of generalizing the Standard Model was examined by Gelmini and Roulet [7] and Boehm and Vogel [8]. Here we attempt to do this in the most pictorial way possible.

Let us assume, for instance, that the neutrino polarization is not $100 \%$ or that the lepton number conservation is violated or that there is an admixture of weak right-handed $(\mathrm{V}+\mathrm{A})$-currents. What then happens to the neutrino mass: will it remain zero (as in the Standard Model), or will it become nonzero? Let us examine both possibilities, starting with the case of $m_{v} \neq 0$, from the standpoint of the theories of Dirac and Majorana (we will speak of the Dirac and Majorana neutrino masses, respectively). According to Dirac's theory (initially developed in 1928 for the electron), generally (i.e. irrespective of the variant of the theory of the weak interaction realized in nature) there can be four types of massive neutrino and antineutrino, $v^{L}, v^{R}, \tilde{v}^{L}$, and $\tilde{v}^{R}$, which differ in lepton number sign $\left(L=+1\right.$ for $v^{L}$ and $v^{R}$, and $L=-1$ for $\tilde{v}^{L}$ and $\tilde{v}^{R}$ ) and helicity sign (left-handed for $v^{L}$ and $\tilde{v}^{L}$, and right-handed for $v^{R}$ and $\tilde{v}^{R}$ ). The equations of motion of the Dirac neutrinos and the corresponding Lagrangian are $\mathrm{C}$-, $\mathrm{P}$-, and $\mathrm{CP}$-invariant and are also Lsymmetric (this symmetry is responsible for the lepton

$\dagger$ If the neutrino had a mass of several electron volts and a lifetime comparable to that of the Universe, they could constitute the majority of matter in the Universe. number conservation). Lorentz transformations offer possibilities for transitions of the type $v^{L} \leftrightarrow v^{R}$ and $\tilde{v}^{R} \leftrightarrow \tilde{v}^{L}$ (this is conceivable if $m_{v} \neq 0$ ), and the CPT-theorem is responsible for transitions of the type $v^{L} \leftrightarrow \tilde{v}^{R}$ and $v^{R} \leftrightarrow \tilde{v}^{L}$. The magnetic moment of the Dirac neutrino differs from zero and, according to the Standard Model, is equal to

$$
\mu_{v} \approx 3 \times 10^{-19} \frac{m_{v}}{1 \mathrm{eV}} \mu_{\mathrm{B}} .
$$

According to Majorana's theory [61] developed in 1937, the neutrino and antineutrino are truly neutral particles, i.e. the neutrino is identical to the antineutrino $\left(\tilde{v}_{\mathrm{e}} \equiv v_{\mathrm{e}}\right)$, but there are two types of neutrinos: left-handed $\left(v_{\mathrm{e}}^{L} \equiv \tilde{v}_{\mathrm{e}}^{L}\right)$, and right-handed $\left(v_{\mathrm{e}}^{R} \equiv \tilde{v}_{\mathrm{e}}^{R}\right)$, which differ in the sign of their helicity. Transitions between these two types of neutrino are possible either through a Lorentz transformation (since $m_{v} \neq 0$ ) or via CPT-inversion. The magnetic moment of the Majorana neutrino is zero. The equation and Lagrangian of its free motion are C-, P-, and CP-invariant. The lepton number is not conserved. What does experiment say about all this?

Earlier we saw that the chief difference between the two theories (in the aspects we are interested in) is that in Dirac's theory $\tilde{v} \neq v$ (in lepton number and helicity) and lepton number conservation is realized, while in Majorana's theory $\tilde{v} \equiv v$ and the lepton number is not conserved, but there are two types of neutrinos with different helicities.

Several experimental estimates have been made of the nonidentity of $v_{\mathrm{e}}$ and $\tilde{v}_{\mathrm{e}}$, i.e. the difference from zero of the overlap of wave functions for the electron neutrino and antineutrino, $\left|\Psi_{\tilde{v}_{\mathrm{e}}} \Psi_{v_{\mathrm{e}}}\right|^{2}$. The results of Davis's experiment [22] mentioned earlier imply that $\left|\Psi_{\tilde{v}_{\mathrm{e}}} \Psi_{v_{\mathrm{e}}}\right|^{2} \approx 0.05$, i.e. differs from zero quite strongly. The value of $\left|\Psi_{\tilde{v}_{\mathrm{e}}} \Psi_{v_{\mathrm{e}}}\right|^{2}$ obtained from accelerator experiments on the $\mathrm{K}^{+} \rightarrow \mathrm{e}^{+} v_{\mathrm{e}} \pi^{0}$ decay yield a figure roughly ten times smaller. But a much stronger indication in favour of $\tilde{v}_{\mathrm{e}} \neq v_{\mathrm{e}}\left(\left|\Psi_{\tilde{v}_{\mathrm{e}}} \Psi_{v_{\mathrm{e}}}\right|^{2}=10^{-12}\right)$ is the absence of any positive result from numerous and prolonged searches for the double neutrinoless beta decay (the $2 \beta 0 \mathrm{v}$ decay) allowed by Majorana's theory:

$$
2 \mathrm{n} \rightarrow 2 \mathrm{p}+2 \mathrm{e}^{-}
$$

which in Dirac's theory is forbidden by lepton number conservation. The latter theory allows only for a twoneutrino double beta decay (the $2 \beta 2 v$-decay), which proceeds with lepton number conservation according to the following scheme

$$
2 \mathrm{n} \rightarrow 2 \mathrm{p}+2 \mathrm{e}^{-}+2 \tilde{v}_{\mathrm{e}}
$$

(for more details see Section 4).

If the $2 \beta 0 \mathrm{v}$-decays were discovered, we could say that the electron neutrino is of the Majorana type, which would lead to an estimate of its mass. But the $2 \beta 0 \mathrm{v}$-decay has not been discovered, and so the controversy between the Dirac and Majorana theories is yet to be resolved. The difference between $v_{\mathrm{e}}$ and $\tilde{v}_{\mathrm{e}}$ observed in experiments can be attributed (irrespective of whether the lepton number is conserved) to a difference in the helicities, which exists in both theories and contributes to overlapping of wave functions on a scale of $\left(m_{v} / E_{v}\right)^{2}$.

Concluding this discussion of the case where $m_{v} \neq 0$, we note that when $m_{v} \neq 0$, both theories allow for neutrino oscillations, although this condition is necessary but not sufficient (for more details see Section 5). 
Let us now examine the case where $m_{v}=0$. In Dirac's theory the neutrino is massive $\left(m_{v} \neq 0\right)$. But if we add Pparity nonconservation and the absence of $\mathrm{C}$-invariance with concurrent $\mathrm{CP}$-invariance conservation to the theory, it becomes the two-component theory of longitudinally polarized neutrinos with $m_{v}=0$, or Weyl's theory [62]. In this theory $v_{v} \equiv c$, i.e. helicity is an intrinsic property of the neutrino and is independent of the reference frame. The neutrino always has left-handed helicity and the antineutrino, right-handed helicity. Transitions between $v$ and $\tilde{v}$ are forbidden not only because the lepton number must be conserved but also because helicity must not change, i.e. in this theory the $2 \beta 0 \mathrm{v}$-decay comes about as if it is doubly forbidden, $\left|\Psi_{\tilde{v}} \Psi_{v}\right|^{2} \equiv 0$. Neutrino oscillations are also forbidden, since they require that $m_{v} \neq 0$.

In Majorana's theory, the assumption that $m_{v}=0$ would seem to forbid both oscillations and the $2 \beta 0 \mathrm{v}$-decay (in the latter in helicity) as well. However, according to an existing viewpoint (e.g., see Refs [12, 63]), if one allows for an admixture of right-handed currents in weak interactions, then the $2 \beta 0 \mathrm{v}$-decay becomes possible no matter how small the neutrino mass is, which is another way of saying that $m_{v}=0$. In this case the admixture of right-handed currents can interact with the polarized clockwise neutrino emitted in the first act of $2 \beta$-decay, which makes the second act possible (neutrino capture by the second neutron). The $2 \beta 0 v$-decay with $m_{v}=0$ will differ from the $2 \beta 0 v$-decay with $m_{v} \neq 0$ in the nature of the single-electron spectrum in the $2 \beta$-decay.

Summarizing, we arrive at the following conclusions.

1. As yet, we do not know there we are dealing with the Dirac neutrino or the Majorana neutrino, but the problem can be resolved if $2 \beta 0 v$-decay is discovered. The other method, an experimental estimation of the neutrino magnetic moment, is unlikely to produce any result in view of the smallness of the Dirac value of this moment.

2. The neutrino mass $\left(m_{v}=0\right.$ or $\left.m_{v} \neq 0\right)$ is still unknown as well, but an attempt can be made to find it either directly, using experiments on studying the beta spectrum near its edge, or indirectly, by discovering neutrino oscillations (for $m_{v} \neq 0$ ) or the $2 \beta 0 v$-decay (for $m_{v_{\mathrm{e}}} \neq 0$ and $m_{v_{\mathrm{e}}}=0$ ).

3. The cases where Majorana neutrinos have $m_{v_{\mathrm{e}}}=0$ or $m_{v_{\mathrm{e}}} \neq 0$ can be distinguished by the nature of the energy and angular spectra for single electrons of the $2 \beta$-decay.

4. Even if a direct experiment reveals that $m_{v} \neq 0$, this does not mean that the $2 \beta 0 v$-decay exists (for which the neutrino must be of the Majorana type) nor does it mean that neutrino oscillations occur (such oscillations require that $m_{1} \neq m_{2}$ ).

One more remark is in order. The case of $m_{v} \neq 0$ would suit astrophysicists perfectly, since in 1980 they provided the following bound on the sum of all three neutrino masses [64]:

$$
\sum m_{v}=m_{v_{\mathrm{e}}}+m_{v_{\mu}}+m_{v_{\tau}}<40 \mathrm{eV}
$$

(compare this value with the experimental data listed in Sections 3.2 and 3.3, according to which $\sum m_{v}<24 \mathrm{MeV}$ ).

In 1995 more stringent bounds on the neutrino mass were set, which followed from the need to explain the solar neutrino deficit and the atmospheric neutrino anomaly (see Section 5). As noted by Weinheimer [65], who cited K Kleinknecht (unpublished), both problems can be solved if we assume that there exist oscillations of the type $v_{\mathrm{e}} \leftrightarrow v_{\mu}$ and $v_{\mu} \leftrightarrow v_{\tau}$ with such a parameter $\Delta m^{2}$ that makes it possible to obtain (in what is known as the see-saw mechanism) an upper bound on the three basic neutrinos $v_{1}, v_{2}$ and $v_{3}$ :

$$
m_{v_{1}} \leqslant 2 \times 10^{-4} \mathrm{eV} ; \quad m_{v_{2}} \leqslant 3 \times 10^{-3} \mathrm{eV} ; \quad m_{v_{3}} \leqslant 10^{-1} \mathrm{eV} .
$$

Another current estimation originates from an attempt to explain the difficulties of the model of hot dark matter. For this, the mass of the electron neutrino should be about $2 \mathrm{eV}$ [65], a value approached by current experimental findings (see Sections 3.2.2 and 3.2.3).

Extremely interesting predictions concerning the mass of Majorana neutrinos arise from studies on the fairly recently suggested branches of the $2 \beta 0 \mathrm{v}$-decay involving a hypothetical particle, the majoron $\mathrm{M}^{0}$ (see Section 4).

\subsection{Estimates of the $v_{\mathrm{e}}$ mass from the analysis of the beta spectrum of tritium and observations of the Supernova 1987A burst}

3.2.1 The idea of estimating the neutrino mass from analysis of the beta spectrum of tritium. Pauli's hypothesis on the existence of a neutrino carrying off the missing energy in beta decay implies that to within a negligible (for modern experimental errors) recoil energy the energy $E_{0}$ of beta decay is

$$
\begin{aligned}
E_{0} & =M(A, Z)-M(A, Z+1)-m_{\mathrm{e}} \\
& =E_{\mathrm{e}}+E_{v}=E_{\mathrm{e}}^{\max }+m_{v} c^{2},
\end{aligned}
$$

where $M(A, Z)$ and $M(A, Z+1)$ are the masses of the initial and final nuclei, $m_{\mathrm{e}}$ is the electron mass, $E_{\mathrm{e}}$ is the electron kinetic energy, $E_{v}$ is the total neutrino energy, and $m_{v} c^{2}$ is the neutrino mass.

What is also obvious is that the lower the energy $E_{0}$, the more precise are the estimates of the neutrino mass $m_{v}$. For this reason, the common approach to estimating the neutrino mass is to analyze the beta spectrum of tritium ${ }_{1}^{3} \mathrm{H}$, which has $E_{0} \approx 0.0186 \mathrm{MeV}$ and a recoil energy lower than $0.6 \mathrm{eV}$.

The idea of the method (suggested by Fermi) consists in studying the right edge of the electron spectrum near its boundary. It occurs that for $m_{v} \equiv 0$ the curve representing spectrum asymptotically tends to zero, while for $m_{v} \neq 0$ the curve rapidly falls of at a distance $m_{v} c^{2}$ from $E_{0}$. Let us study the reasoning behind these conclusions [2].

According to Fermi's theory, the shape of the electron spectrum is determined by the statistical factor $p_{\mathrm{e}} E_{\mathrm{e}} p_{v} E_{v}$ (here $p_{\mathrm{e}}$ and $p_{v}$ are the electron and neutrino momenta, respectively):

$$
\begin{aligned}
N\left(E_{\mathrm{e}}\right) & \sim p_{\mathrm{e}} E_{\mathrm{e}} p_{v} E_{v}\left[\left(E_{0}-E_{\mathrm{e}}\right)^{2}-m_{v}^{2} c^{4}\right]^{1 / 2}\left(E_{0}-E_{\mathrm{e}}\right) \\
& =p_{\mathrm{e}} E_{\mathrm{e}}\left(E_{0}-E_{\mathrm{e}}\right)^{2} \sqrt{1-\frac{m_{v}^{2} c^{4}}{\left(E_{0}-E_{\mathrm{e}}\right)^{2}}}
\end{aligned}
$$

If $m_{v} \equiv 0$, the above expression is simplified and we arrive at

$$
N\left(E_{\mathrm{e}}\right) \sim p_{\mathrm{e}} E_{\mathrm{e}}\left(E_{0}-E_{\mathrm{e}}\right)^{2},
$$

which shows that as $E_{\mathrm{e}}$ grows the electron spectrum does indeed tend to zero and reaches it at $E_{\mathrm{e}}=E_{0}$ (Fig. 1a).

Similarly, differentiating (14) at $m_{v} \neq 0$, we see that it has a vertical derivative at $E_{\mathrm{e}}=E_{\mathrm{e}}^{\max }=E_{0}-m_{v} c^{2}$ (Fig. 1b). In practice, however, to compare the results with experimental data it is more convenient to use what is known as the Curie 

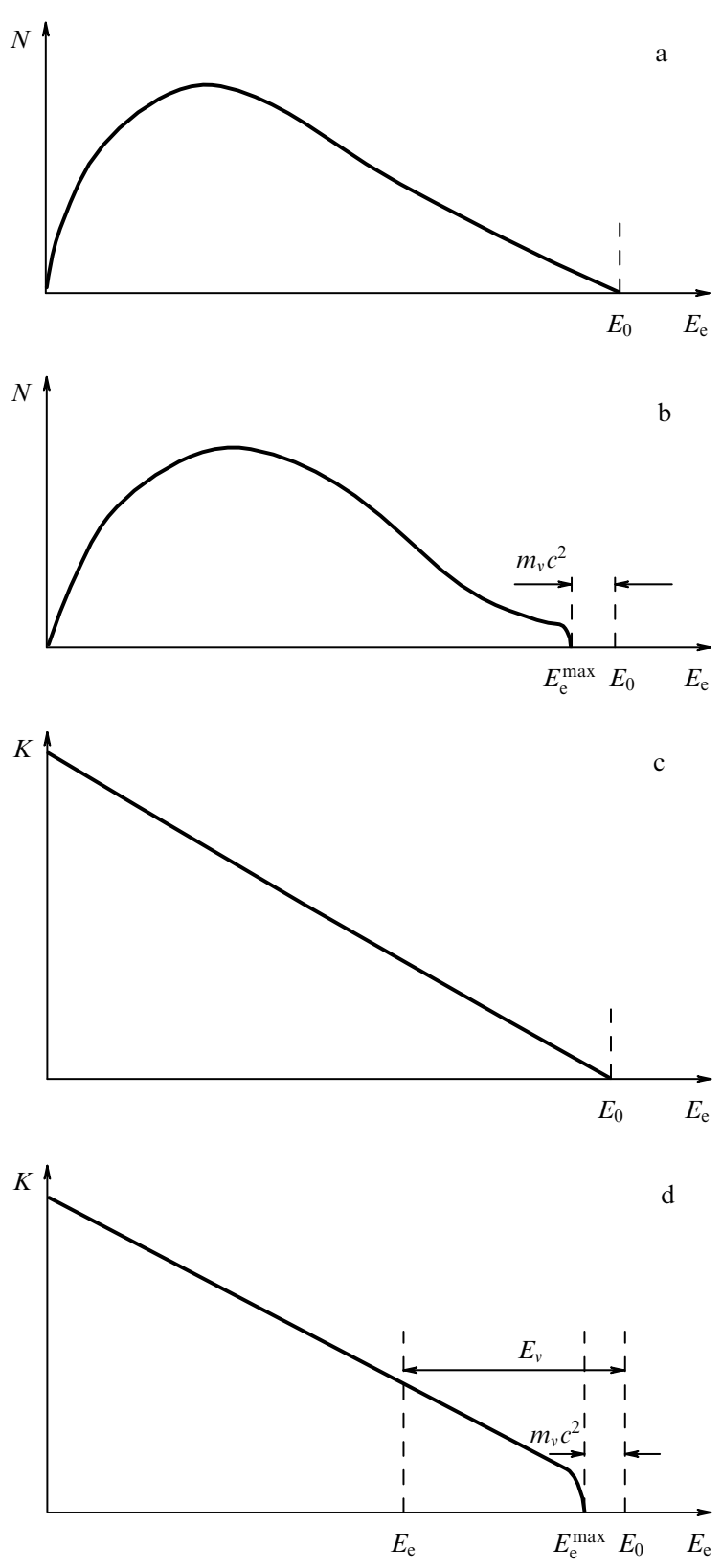

Figure 1. The beta spectra for $m_{v}=0$ (a) and $m_{v} \neq 0(\mathrm{~b})$, and the respective Curie plots for $m_{v}=0$ (c) and $m_{v} \neq 0$ (d).

plot:

$$
K\left(E_{\mathrm{e}}\right)=\left(\frac{N\left(E_{0}\right)}{p_{\mathrm{e}} E_{\mathrm{e}}}\right)^{1 / 2} .
$$

Comparison of (15) and (16) at $m_{v} \equiv 0$ shows that $K\left(E_{\mathrm{e}}\right) \sim\left(E_{0}-E_{\mathrm{e}}\right)$, i.e. the Curie plot is a straight line intersecting the energy axis at $E_{\mathrm{e}}=E_{0}$ (Fig. 1c). For $m_{v} \neq 0$, the Curie plot is given by the following expression:

$$
K\left(E_{\mathrm{e}}\right) \sim\left(E_{0}-E_{\mathrm{e}}\right) \sqrt[4]{1-\left(\frac{m_{v} c^{2}}{E_{0}-E_{\mathrm{e}}}\right)^{2}},
$$

and differentiating it again leads to a vertical derivative at $E_{\mathrm{e}}=E_{\mathrm{e}}^{\max }$. Thus, in this case the Curie plot is almost entirely a straight line except for the right end, where it intersects the energy axis at right angles at a point that is $m_{v} c^{2}$ distant from $E_{0}$ (Fig. 1d).
Of course (as is always the case), the Curie plots in Figs 1c and $1 \mathrm{~d}$ represent an idealized picture, which in experimental conditions becomes highly distorted because of the finite resolution of the spectrometer, scattering and absorption effects, and the superimposition of spectra of the transitions into excited states of a finite nucleus, Coulomb shifts, etc. All these factors are reflected by corrections in the theoretical formula and makes the curve considerably less pronounced than in Fig. 1d, which considerably hampers the estimation of the neutrino mass, especially if its possible value is close to zero. And, of course, what complicates situation still more is that at the spectrum edge there are very few useful electrons compared to the background.

What actually happens is that the researcher is forced to do many measurements of the shape of the spectrum near the edge and then compare the results with those of corrected theoretical formulae obtained for various neutrino masses, different values of $E_{0}$ (within the experimental scatter $E_{0} \pm \Delta E_{0}$ ), different energies of excitation of a finite nucleus, etc. The results are then processed according to the $\chi^{2}$-criterion.

3.2.2 Estimate of the mass of $\tilde{v}_{\mathbf{e}}$ from the beta spectrum of tritium. The first results of studying the beta spectrum of tritium ${ }_{1}^{3} \mathrm{H}$ appeared in 1949, when Hanna and Pontecorvo [66] obtained a value of $1 \mathrm{keV}$ for the upper bound on the neutrino mass. They used a proportional counter filled with gaseous tritium. In 1952, Langer and Moffat [67] investigated the beta spectrum of tritium using a magnetic spectrometer and obtained a value of $250 \mathrm{eV}$ for an upper bound on the neutrino mass. They employed a thin $\left(0.5 \mu \mathrm{g} \mathrm{cm}^{-2}\right)$ source which was prepared by evaporating succinic acid saturated with tritium.

Further progress in estimating an upper bound on the neutrino mass was related to improvements in the magnetic spectrometer technique. Using this approach, Bergkvist [68] found in 1972 that $m_{v}<55 \mathrm{eV}$, and Tretyakov et al. [69] (Institute of Theoretical and Experimental Physics, Moscow) obtained in 1976 a then-record value of $m_{\tilde{v}_{e}}<35 \mathrm{eV}$.

An entirely new step was taken in $1980-1981$ by Lubimov et al. [70] (Institute of Theoretical and Experimental Physics, Moscow). By introducing additional improvements into the magnetic spectrometer they were able to obtain not only an upper bound on the neutrino mass but a lower bound as well:

$$
14 \mathrm{eV} \leqslant m_{v} c^{2} \leqslant 46 \mathrm{eV},
$$

and refined the value of $E_{0}=18577 \pm 13 \mathrm{eV}$.

The basic difficulty that these researchers encountered was related to the use of tritium-doped valine as a source of electrons instead of tritium, since generally tritium-doped valine can distort the spectrum. As a result of studying this problem, Boris et al. [71] arrived at new bounds on the neutrino mass:

$$
17 \mathrm{eV} \leqslant m_{v} c^{2} \leqslant 40 \mathrm{eV} .
$$

In 1986, Fritschi et al. [72] of Zurich obtained the first result that did not agree with the results of Lubimov et al. [70] and Boris et al [71]. They used a Tretyakov type magnetic spectrometer [73] with a toroidal magnetic field and a radial electrostatic retarding field surrounding the source, which was a layer of tritium deposited on a thin carbon substrate. At the spectrometer exit, before entering the counter, the electrons were retarded by a voltage of $15 \mathrm{kV}$. 
A somewhat modified formula of type (14), which allowed for all the final electron states, the background, and other factors, was used in processing the beta spectrum. As a result, an upper bound on the neutrino mass was found to be

$$
m_{v} c^{2}<18 \mathrm{eV},
$$

which does not contradict the value $m_{v}=0$.

In the research that followed, carried out by other groups from Tokyo, Zurich, Los Alamos, Livermore, and Mainz, the values of an upper bound for the neutrino mass were lowered to 13.0 [74], 11.0 [75], 9.3 [76], 8.0 [77], and $7.2 \mathrm{eV} \mathrm{[14].}$

Thus, the results of Lubimov et al. [70] and Boris et al. [71] were not confirmed, but they played an extremely important role by stimulating research in the beta spectrum of tritium up to its edge with ever growing accuracy. In particular, Robertson et al. [76] were the first to use molecular tritium $\mathrm{T}_{2}$ as a gaseous source, where a molecule is a two-electron system, which is much simpler for calculations than the manyelectron molecules of solid sources.

Below we discuss in greater detail two of the latest works in which analysis of the beta spectrum of tritium is used to arrive at record-breaking estimates of the upper bound on the electron antineutrino mass.

3.2.3 The Mainz University neutrino experiment. At a conference held in 1995 in Osaka, Japan, devoted to weak and electromagnetic interactions in nuclei, Weinheimer [65] gave a talk that consisted of a brief review of the results of previous (1991) measurements [14] that yielded $m_{v} c^{2}<7.2 \mathrm{eV}$, and reported about a new result, $m_{v} c^{2}<6.0 \mathrm{eV}$.

Weinheimer and collaborators thoroughly studied a narrow boundary section of the beta spectrum of tritium. The measurements were made by a novel spectrometer based on the idea of using a combination of an axial magnetic channel and a retarding electrostatic field. The spectrometer adiabatically transforms the kinetic energy of the cyclotron electron motion around the magnetic field lines into the longitudinal energy of the analyzing electric field. A device constructed in the manner has an exceptionally high resolution and transmittance, which makes it possible to study a narrow segment of the spectrum near its end with a high signal-to-noise ratio.

For a source, the researchers used a film of molecular tritium 40 monolayers thick (with an activity of $4 \mathrm{mCi}$ ) condensed (by freezing) on an aluminium (in 1991) or graphite (in 1994) substrate. The measured counting rate $\mathrm{d} N\left(E_{\mathrm{e}}\right) / \mathrm{d} t$ was approximated by the following theoretical formula (which can easily be derived from (14) after the factors mentioned below are taken into account):

$$
\begin{aligned}
\frac{\mathrm{d} N\left(E_{\mathrm{e}}\right)}{\mathrm{d} t}= & \left(A F \sum_{i} W_{i} \varepsilon_{i} \sqrt{\varepsilon_{i}^{2}-m_{i}^{2} c^{4}}\right) \\
& \times T F \times E_{\mathrm{e}} L \times B S+B G,
\end{aligned}
$$

where $A$ is the amplitude, $F$ is the Fermi function, $W_{i}$ is the relative amplitude of the transition into the $i$ th excited final state with an excitation energy $V_{i}$, and then $\varepsilon_{i}=E_{0}-V_{i}-E_{\mathrm{e}}$ is the segment of the spectrum extending to the final point, $T F$ is the transmittance function of the spectrometer, $B S$ represents the background scattering in the source substrate, $E L$ describes the energy losses in the source, and, finally $B G$ is a function representing the background.
The structure of (21) implies that the experimental estimate of the neutrino mass involves $m_{v}^{2}$, which in principle (in view of experimental errors and the theoretical analysis) can assume not only positive values but also negative (nonphysical) values $m_{v}^{2}<0$. In particular, the final result of Weinheimer et al. [14] obtained in 1991, is

$$
m_{v}^{2} c^{4}=-39 \pm 34_{\text {stat }} \pm 15_{\text {syst }} \mathrm{eV}^{2},
$$

which yielded the following estimate of $m_{v}$ :

$$
m_{v} c^{2}<7.2 \mathrm{eV}(95 \% \text { confidence level })
$$

Elaborating on the reasons for the emergence of nonphysical negative values of $m_{v}^{2}$, the authors believe that such values appear because of some sort of systematic error, which leads to an excessive counting rate.

Using a higher quality source in their 1994 measurements, the researchers were able to double the volume of data gathered, so that even at a distance of $13 \mathrm{eV}$ below the final point $E_{0}$ the signal was clearly visible above the background (Fig. 2a). The analysis leaned upon the last $143 \mathrm{eV}$ segment before the end point. It was found that in the vicinity of the final point the values of $m_{v}^{2} c^{4}$ were stable with respect to the fitting intervals used in calculations and were compatible with zero. The final result was (Fig. 2b)

$$
m_{v}^{2} c^{4}=-22 \pm 17_{\text {stat }} \pm 17_{\text {syst }} \mathrm{eV}^{2},
$$
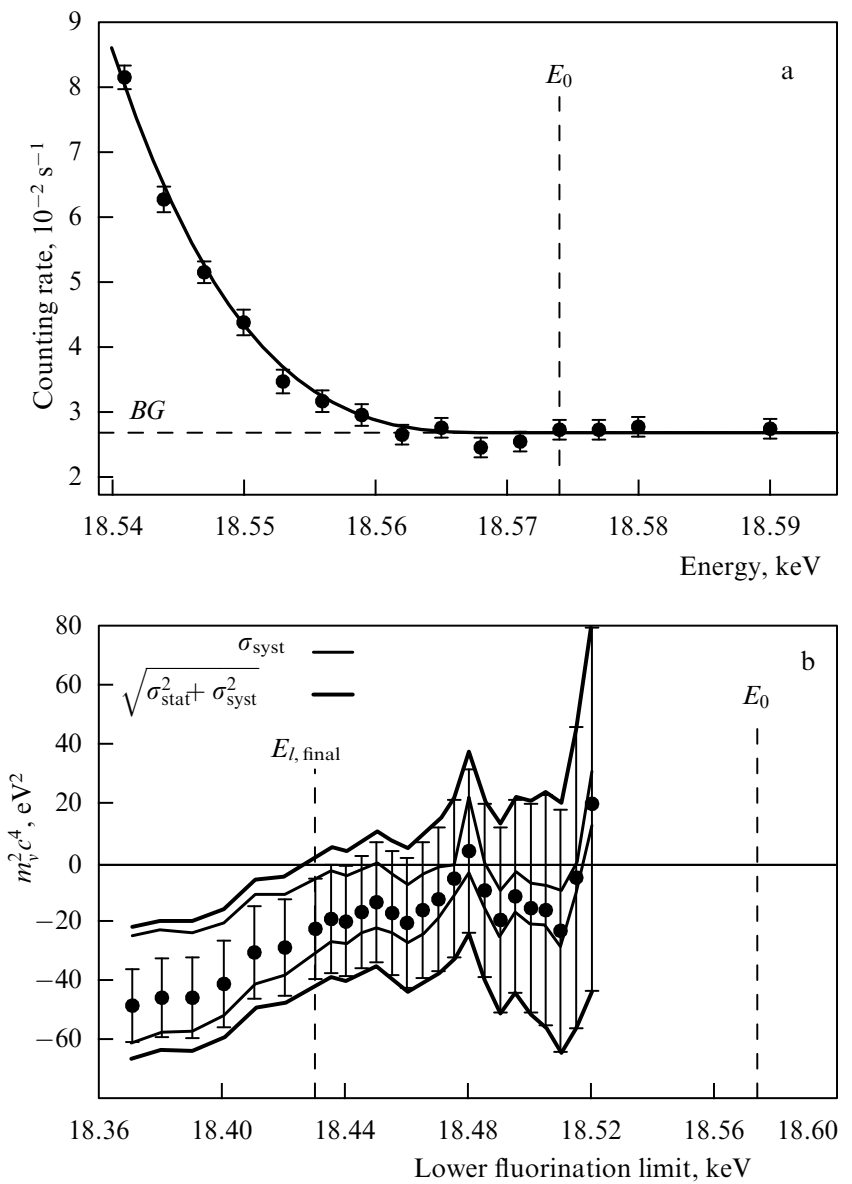

Figure 2. Results of the Mainz group: (a) the segment of the beta spectrum near the edge, and (b) the result for $m_{v}^{2} c^{4}$. 
which is much better than the 1991 result (22) and yields the following upper bound on the mass of $\tilde{v}_{\mathrm{e}}$ :

$$
m_{v} c^{2}<6.0 \mathrm{eV}(95 \% \text { c.1.). }
$$

In the future, Weinheimer and his collaborators intend to reduce the background noise of the device considerably, which will enable them to lower an upper bound on the electron antineutrino mass down to $2 \mathrm{eV}$, but even today the value attained of $m_{v} c^{2}<6.0 \mathrm{eV}$ is a record.

3.2.4 The Troitsk facility neutrino experiment. The second record-breaking (and even smaller) value of an upper bound on the mass of $\tilde{v}_{\mathrm{e}}\left(m_{\tilde{v}_{\mathrm{e}}}<4.35 \mathrm{eV}\right)$ was obtained by Belesev et al. [15] and Lobashev [78] at the Institute for Nuclear Research of the Russian Academy of Sciences in the city of Troitsk (Moscow region). The main idea of this experiment was suggested by Lobashev and Spivak [79], and the experimental devices are described in Ref. [80]. A distinctive feature of this experiment is the use of an integral electrostatic spectrometer with adiabatic magnetic collimation and a gaseous tritium source.

The schematic of the experimental device is depicted in Fig. 3 [80]. The essential components of the spectrometer and the gaseous tritium source are several superconducting solenoids generating a continuous longitudinal magnetic field through the entire device. The field strength varies between 1 and $5 \mathrm{~T}$ inside the source and increases up to $8 \mathrm{~T}$ at the entrance to the spectrometer. After the entrance it drops to $1 \mathrm{mT}$ in the median section of the spectrometer and, finally, grows to $2.6 \mathrm{~T}$ near the detector.

The cylindrical electrode in the central part of the detector served as an integral electrostatic analyzer. All the magnetic and electric fields were calculated and adjusted to ensure adiabatic motion of the electrons through the source and spectrometer along the magnetic field lines into the electron detector.

A lithium-coated drift silicon counter $20 \mathrm{~mm}$ in diameter was used as the detector in the device. Since it detected only electrons that had travelled along field lines, the background noise from the decay of tritium atoms adsorbed by the walls of the spectrometer and the source was practically zero.
Gaseous tritium was injected into the middle of a threemeter tube placed inside the solenoids, evacuated from both ends of the pipe, purified, and again injected into the pipe, thus circulating continuously over the course of two weeks with portions of the fresh gas regular added to the system. A typical gaseous mixture was $\mathrm{T}_{2}+\mathrm{HT}+\mathrm{H}_{2}$ with a ratio of 6:8:2.

The spectrometer's resolution was found by the formula

$$
\Delta E_{\mathrm{e}}=E_{\mathrm{e}} \frac{H_{\min }}{H_{\max }},
$$

where $E_{\mathrm{e}}$ is the electron energy, $H_{\max }$ is the magnetic field strength at the point of solenoid entry into the spectrometer, and $H_{\min }$ is the magnetic field strength in the median section of the spectrometer [79]. For most measurements the resolution was $3.7 \mathrm{eV}$. At $E_{\mathrm{e}}=15-19.5 \mathrm{keV}$, a high voltage of $18.7 \mathrm{kV}$, and the rated magnetic field, the background noise produced by the detector amounted to $5-7 \mathrm{mHz}$, while for the tritium source operating at full intensity this background amounted to $15-25 \mathrm{mHz}$. The vacuum was maintained at $10^{-9}$ Torr.

The measurements were made with the spectrometer's potential varying between 18000 and $18770 \mathrm{~V}$. The spectrometer's resolution function was measured by a photoemission electron gun with an ultraviolet lamp.

Figure 4 depicts a portion of the measured spectrum and the Curie plot near the right end of the spectrum. Notice that the first statistically significant point in the spectrum appears at a distance of $6 \mathrm{~V}$ from the effective final point $E_{0}=18570.5 \mathrm{~V}$ of the spectrum. The experimental spectrum was expressed on the scale of the spectrometer's potential, while the fitted theoretical spectrum was expressed on the electron energy scale.

The differential theoretical spectrum for $m_{v}^{2} \geqslant 0$ was calculated by the usual formula [cf. (14) and (21)]

$$
\varepsilon \sqrt{\varepsilon^{2}-m_{v}^{2} c^{4}}
$$

and for $m_{v}^{2}<0$, by the formula

$$
2 \varepsilon^{2}-\varepsilon \sqrt{\varepsilon^{2}-\left|m_{v}^{2}\right| c^{4}}
$$

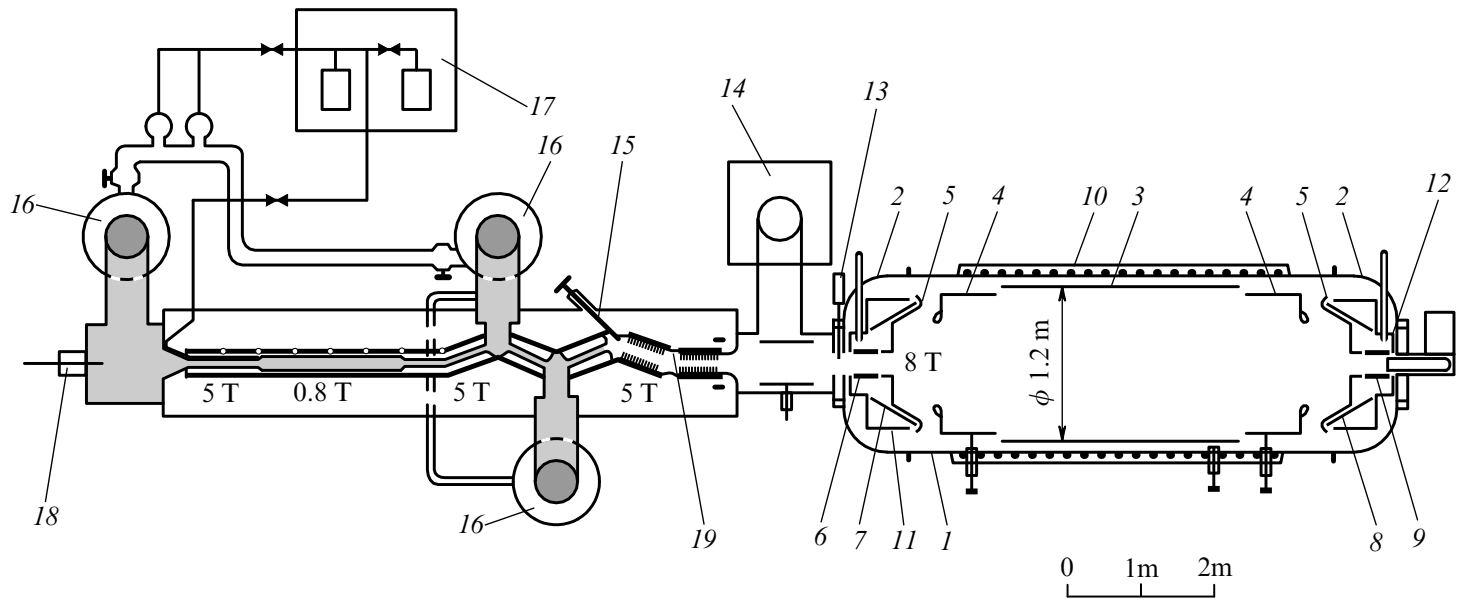

Figure 3. Schematic of the experimental set-up used by the Troitsk group: 1 and 2 , the vacuum chamber; 3 and 4 , the electrostatic analyzer; 5 , the grounded electrode; $6-9$, the superconducting solenoids; 10 , the coil; 11 , the nitrogen screen; 12 , the detector; 13 , the high-speed shutter; 14 , the titanium pump; 15 , the cold valve; 16 , the mercury diffusion pump; 17 , the $\mathrm{T}_{2}$ purification system; 18 , the electron gun; and 19 , the argon pump. 


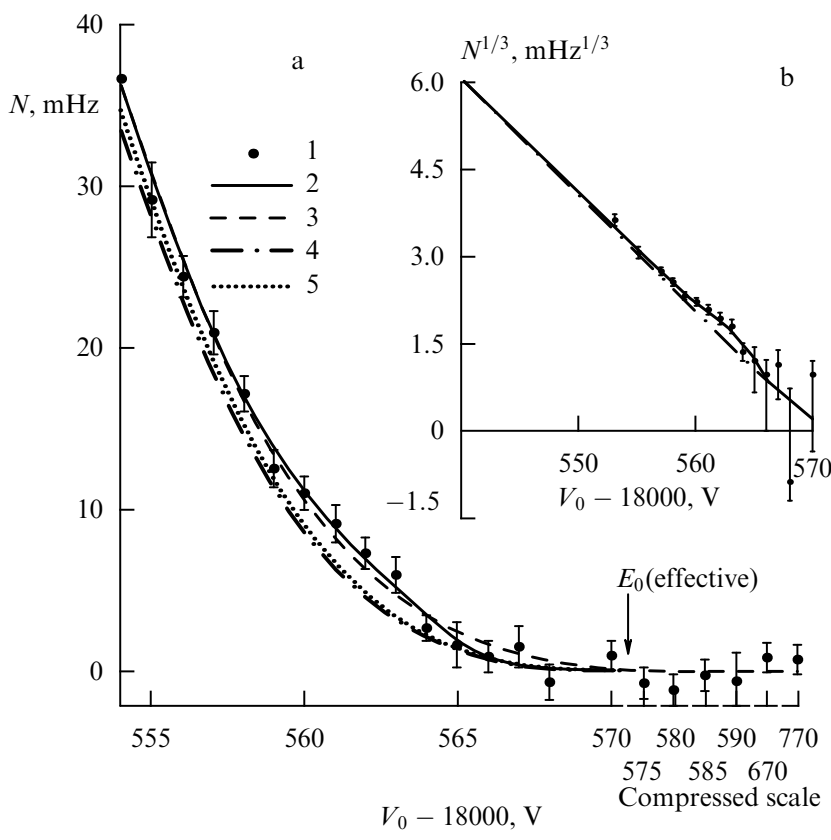

Figure 4. Part of the tritium spectrum (a) and the Curie plot (b) near the right edge (results obtained at the facility in Troitsk): $V_{0}$ is the spectrometer voltage, $N_{1}$ is the experimental spectrum, and $N_{2}$ to $N_{5}$ are the theoretical spectra with different variables.

where $\varepsilon=E_{0}-E_{\mathrm{e}}, E_{\mathrm{e}}$ is the electron energy, and $E_{0}$ is the energy at the final point of the spectrum. The structure of both formulae (27) and (28) suggests that at $m_{v}^{2}=0$ they merge.

The fitting procedure consisted in varying four parameters: the normalization factor, the energy of the final point of the spectrum, the value of $m_{v}^{2}$, and the background level. The lower boundary of the fitting interval of the spectrum, $E_{\text {low }}$, varied between 18000 and $18500 \mathrm{~V}$. The upper end was fixed at $18770 \mathrm{~V}$. The results of fitting yielded $m_{v}^{2}<0$ for all values of $E_{\text {low }}$, with $\left|m_{v}^{2}\right|$ increasing when either the upper or lower limit of the selected values of $E_{\text {low }}$ is approached.

In the interval of $E_{\text {low }}$ values from 18300 to $18450 \mathrm{~V}$, the value of $m_{v}^{2}$ is independent of $E_{\text {low }}$, and processing the results obtained yields the best $\chi^{2}$. Only two parameters were varied for this interval, the normalization factor and the final energy. The result was the following value for the mass

$$
m_{v}^{2}=-22 \pm 4.8 \frac{\mathrm{eV}^{2}}{c^{4}}
$$

The researchers noted that the nonphysical values $m_{v}^{2}<0$ are related to the presence of several systematic features (kinks) in the curve in the experimental spectrum, which are ignored in the common calculation of the theoretical spectrum by formula (27). Figure 4 shows that the difference between the experimental and theoretical spectra is concentrated primarily at a distance of $7-15 \mathrm{eV}$ from the final point and is also evident (to a smaller extent) at lower energies. By using formula (28) one can allow for this anomaly and obtain a value of $19.0 \mathrm{eV}^{2} c^{-4}$ for the upper bound on the positive value of $m_{v}^{2}$, which yields

$$
m_{v}<4.35 \frac{\mathrm{eV}}{c^{2}} \quad(95 \% \text { c.l. }) \text {. }
$$

It should be noted, however, that the authors of the widely known tables 'Review of Particle Properties' [21] believe this anomaly to be so important that in the section titled 'Lepton Summary Table' they do not list the latest exact estimates of $m_{v_{\mathrm{e}}}$, since they assume that starting at $m_{v_{\mathrm{e}}}<10-15 \mathrm{eV}$ these values are distorted in measurements.

\subsubsection{Estimate of $m_{v_{e}}$ from observations of the Supernova} $1987 \mathrm{~A}$ burst. In addition to studying the beta spectrum of tritium, there is another way to estimate the mass of $v_{\mathrm{e}}$. In 1968, Zatsepin [81] suggested using the observational data on the gravitational collapse of a star to estimate the neutrino mass. According to the hot Universe model, each cubic centimetre of space contains approximately 300 relic neutrinos with an energy of $10^{-4} \mathrm{eV}$. Against this neutrino background, high-energy (up to $10^{21} \mathrm{eV}$ ) neutrino fluxes are continuously being generated as a result of the interaction between cosmic rays and the interstellar medium [5]. Moreover, in the event of extremely rare explosions of supernovae, the gravitational collapse of the central core of such stars produces extremely powerful fluxes of medium-energy neutrinos $(10-20 \mathrm{MeV})$. The method consists in detecting a sequence of signals of neutrinos of different energies generated by the collapsing star, neutrinos that reach the Earth at various times due to their different velocities. The difference in the arrival times of two neutrinos, $\Delta t_{v}$, that were produced simultaneously with energies $\varepsilon_{1}$ and $\varepsilon_{2}$ and travelled a distance $D$ equals

$$
\Delta t_{v}=\frac{D}{2 c}\left(m_{v} c^{2}\right)^{2}\left(\varepsilon_{1}^{-2}-\varepsilon_{2}^{-2}\right) .
$$

The explosion of the Supernova 1987A was registered on the night of February 23-24, 1987, at the Las Campanas Observatory in Chile operated by Toronto University (Canada) and was then studied in many observatories [16, 82]. At approximately the same time, the researchers at four neutrino laboratories registered 29 neutrino events that could be attributed to the interactions of the neutrinos produced as results of the collapse of this star. The largest number of events was registered in Japan (eight reliable and three questionable) by the Cherenkov water detector Kamiokande (2140 tons of water) [83] at a depth of 2700-m water equivalent in the Kamioka mine. An additional eight events were registered in the US by the IMB (Irvin-MichiganBrookhaven) Cherenkov water detector (5000 tons of water) in a salt mine under Lake Erie at a depth of 1570-m water equivalent [84]. Finally, five events each were registered by the LSD (Liquid Scintillation Detector) facility with a mass of 90 tons of liquid scintillator in the Mont Blanc Tunnel connecting Chamonix, Mont Blanc, France, and Courmayeur, Italy, at the Russian-Italian underground observatory $(5200-\mathrm{m}$ water equivalent $[85,86])$, and by the underground BST unit (Baksan Scintillation Telescope, 200 tons of liquid scintillator) at a depth of $850-\mathrm{m}$ water equivalent in Ciscaucasia (North Caucasia) near Mt. Elbrus [87, 88].

In Imshennik and Nadezhin's review [16], devoted to SN 1987A, there appears an estimate of the upper bound on the neutrino mass obtained from the data on the neutrino signal from the burst of this supernova:

$$
m_{v_{\mathrm{e}}} c^{2}<23-28 \mathrm{eV} .
$$

The estimate involved is based on the assumption that the duration of the neutrino signal in the collapse of the star was 
$10-15 \mathrm{~s}$ and that the characteristic neutrino energies were 10 to $20 \mathrm{MeV}$ (the distance between SN 1987A and the Earth is known to a high accuracy).

Of the other results obtained in the studies of the SN 1987A burst [16] we must mention the estimate of the neutrino electric charge $q_{v_{\mathrm{e}}}<10^{-17} e$ and the bound on the magnetic moment, $\mu_{v_{\mathrm{e}}} \leqslant 10^{-14}-10^{-12} \mu_{\mathrm{B}}$, where $\mu_{\mathrm{B}}$ is the electron Bohr magneton.

Notice that extracting data from the neutrino burst of SN 1987A is extremely difficult because of insufficient knowledge of the collapse mechanism, the small number of registered events ( 29 in four detectors), and the poor synchronization of the various detectors in between. In this connection it must be noted that the data gathered from the Kamiokande detector yield an upper bound on the $m_{v_{\mathrm{e}}}$ that is somewhat lower than the values provided by Bahcall and Glashow [89] and Arnett and Rosner [90]:

$$
m_{v_{e}}<10-20 \mathrm{eV} \text {. }
$$

Today new, more sensitive detectors are being built. Among them are the Superkamiokande, in the mountains west of Tokyo [91], and the Sudbury Neutrino Observatory (SNO) [92], and they will be synchronized (see Section 6).

\subsection{Estimates of the masses, spins, and helicities of the muon neutrino $\boldsymbol{v}_{\boldsymbol{\mu}}$ and the tau neutrino $\boldsymbol{v}_{\tau}$}

The exact values of the mass of $v_{\mu}$, and the more so of $v_{\tau}$, have yet to be found, but rough estimates do exist. An estimate of the mass of $v_{\mu}$ has been obtained from the two-particle decay scheme $\left(\pi^{+} \rightarrow \mu^{+}+v_{\mu}\right)$, in which the pion and muon masses and the muon's kinetic energy (momentum) are known. Today the generally accepted value of $m_{v_{\mu}}$ is [21]

$$
m_{v_{\mu}}<0.17 \mathrm{MeV}(90 \% \text { c.l.) } .
$$

There is a certain fine point in this method of estimating $m_{v_{\mu}}$, and ignoring it distorts the value of $m_{v_{\mu}}$. If the $(\pi \rightarrow \mu)$ decay occurs in a state of rest, the laws of conservation of total energy and momentum, $m_{\pi}=E_{\mu}+E_{v_{\mu}}$ and $p_{\mu}^{2}=p_{v_{\mu}}^{2}$, readily yield the following estimate of the mass of $v_{\mu}$ :

$$
m_{v_{\mu}}^{2}=m_{\pi}^{2}+m_{\mu}^{2}-2 m_{\pi} \sqrt{m_{\mu}^{2}+p_{\mu}^{2}} .
$$

The sign of the right-hand side of Eqn (35) is extremely sensitive to the value of the pion mass, so that certain values of $m_{\pi}$ could yield (and in some works this actually happened) a nonphysical value for $m_{v_{\mu}}^{2}$. But, of course, the reason for such 'nonphysical' values is much better understood than that for the nonphysical values we encountered in determining $m_{v_{\mathrm{e}}}$ from the beta decay of tritium.

The spin of $v_{\mu}$ (equal to $1 / 2$ ) and the helicity (left-handed for $v_{\mu}$ and right-handed for $\tilde{v}_{\mu}$ ) have been established from the $(\pi \rightarrow \mu)$ - and $(\mu \rightarrow \mathrm{e})$-decay schemes, in which the spins and helicities of all the charged particles are known beforehand from experiments. Knowing the mass of $v_{\mu}$ and the neutrino energy $\left(E_{v} \simeq 29 \mathrm{MeV}\right)$, one can easily establish the extent to which $v_{\mu}$ and $\tilde{v}_{\mu}$ are nonidentical in helicity: $\left|\Psi_{\tilde{v}_{\mu}} \Psi_{v_{\mu}}\right| \simeq\left(m_{v_{\mu}} / E_{v_{\mu}}\right)^{2} \approx 4 \times 10^{-5}$.

The usual way to estimate the upper bound on the mass of the tau neutrino is to use multihadron decay modes of the tau lepton, say $\tau \rightarrow 5 \pi^{ \pm}+v_{\tau}$.

As in the case with $m_{v_{\mu}}$, the mass of $v_{\tau}$ can be estimated from the primary-particle ( $\tau$-lepton) mass and the masses and kinetic energies of the product particles ( $\pi^{ \pm}$-mesons). Using this method, Albrecht et al. [93] estimated the upper bound on $m_{v_{\tau}}$ as $m_{v_{\tau}}<31 \mathrm{MeV}$, and Cinabro et al. [94], $m_{v_{\tau}}<32.6$ $\mathrm{MeV}$.

Another method of estimating the mass of $v_{\tau}$, which consists in using the two-stage process

$$
\mathrm{e}^{+}+\mathrm{e}^{-} \rightarrow \tau^{+}+\tau^{-} \rightarrow 5 \pi^{ \pm}+v_{\tau}+\tau^{\mp},
$$

yields the following value for the upper bound on $m_{v_{\tau}}$ [95]:

$$
m_{v_{\tau}}<23.8 \mathrm{MeV}(95 \% \text { c.l. }) \text {. }
$$

Finally, the mass of $v_{\tau}$ can be estimated by a standard method usually used for determining $m_{v_{\mathrm{e}}}$, i.e. by analyzing the electron spectrum in the tau-lepton decay. Analysis has shown that the $\tau^{-} \rightarrow \mathrm{e}^{-} \tilde{v}_{\mathrm{e}} v_{\tau}$ decay agrees with the (V-A)theory and yields a value of $m_{v_{\tau}}$ coinciding with that generally accepted [21]:

$$
m_{v_{\tau}}<24 \mathrm{MeV}(95 \% \text { c.l. }) \text {. }
$$

Ratio between widths of the two channels of the taulepton decay,

$$
\frac{\Gamma\left(\tau^{-} \rightarrow \pi^{-} v_{\tau}\right)}{\Gamma\left(\tau^{-} \rightarrow \mathrm{e}^{-} \tilde{v}_{\mathrm{e}} v_{\tau}\right)}=0.52 \pm 0.07
$$

which coincides with the theoretical value (0.6) within experimental error, yields $\boldsymbol{\sigma}_{v_{\tau}}=1 / 2$. The most natural helicity of $v_{\tau}$ is the left-handed.

Thus, all three types of neutrino possess $\operatorname{spin} \sigma=1 / 2$, lefthanded helicity, and, most likely (as astrophysical data suggest and as analysis of beta decay of tritium also suggests for $v_{\mathrm{e}}$ ) have extremely small or zero masses.

\section{Double beta decay}

\section{$4.12 \beta 2 v$ - and $2 \beta 0 v$-decay schemes. Their relation to theory and the purpose of the search}

In Section 3.1 we briefly studied double beta decay to the extent needed for discussing the neutrino mass problem. Let us now discuss this type of decay in detail.

Double beta decay is a process in which two electrons (positrons) are simultaneously emitted by a single atomic nucleus. The theory examines two types of double beta decay: two-neutrino double beta $(2 \beta 2 v)$ decay, and neutrinoless double beta $(2 \beta 0 \mathrm{v})$ decay. Both types have been studied for more than 60 years $[96,97]$. Moreover, from the 1960s and especially today, the possibility of double beta decay with emission of the hypothetical particles, majorons $\mathbf{M}^{0}$ (e.g., see Shchepkin's review [12]) is being actively discussed. This hypothetical scheme will be examined at the end of this section.

The $2 \beta 2 v$-decay is allowed in the universal $(\mathrm{V}-\mathrm{A})$-theory of weak interaction and in the Standard Model of electroweak interaction as a second-order effect proceeding according to the scheme

$$
2 \mathrm{n} \rightarrow 2 \mathrm{p}+2 \mathrm{e}^{-}+2 \tilde{v_{\mathrm{e}}} \quad\left(\text { or } 2 \mathrm{p} \rightarrow 2 \mathrm{n}+2 \mathrm{e}^{+}+2 v_{\mathrm{e}}\right),
$$

which satisfies the law of conservation of the electron's lepton number. The neutrino in these schemes differs from the antineutrino $(v \neq \tilde{v})$ by the sign of the lepton number and 
the nature of interaction, while zero and nonzero neutrino and antineutrino masses are allowed: $m_{v}=m_{\tilde{v}} \neq 0$ and $m_{v}=m_{\tilde{v}}=0$ (Fig. 5a).

$2 \beta 0 \mathrm{v}$-decay lies outside the scope of the Standard Model. It is allowed if the law of lepton number conservation is violated and $\tilde{v}_{\mathrm{e}} \equiv v_{\mathrm{e}}$, i.e. the neutrino exhibits a Majorana nature (Fig. 5b). Here, as already noted in Section 3, if the weak charged current is strictly left-handed, then $2 \beta 0 \mathrm{v}$-decay is possible only at $m_{v} \neq 0$. In this case, the first right-handed neutrino has a small admixture $\left(\sim m_{v} / E_{v}\right)$ of left-handed helicity, due to which it can interact with the second neutron. But if the charged lepton current contains an admixture $\eta^{\prime}$ of the right-handed component, i.e.

$$
j_{\mu}=\bar{e} \gamma_{\mu}\left[\left(1+\gamma_{5}\right)+\eta^{\prime}\left(1-\gamma_{5}\right)\right] v,
$$

then the neutrino mass may be as small as desired [12]. In this case the polarized clockwise neutrino emitted by the first neutron can interact with the second neutron due to the admixture of right-handed currents. The simplest scheme of $2 \beta 0 \mathrm{v}$-decay is

$$
2 \mathrm{n} \rightarrow 2 \mathrm{p}+2 \mathrm{e}^{-} \quad\left(\text { or } 2 \mathrm{p} \rightarrow 2 \mathrm{n}+2 \mathrm{e}^{+}\right),
$$

which violates the law of lepton number conservation $\left(\Delta L_{\mathrm{e}}=2\right.$, see Fig. $\left.5 \mathrm{~b}\right)$. Thus, $2 \beta 0 v$-decay offers a sensitive test of lepton number nonconservation, the Majorana nature of neutrinos, and the existence of weak right-handed charged currents.

But how can one detect double beta decay and how is one to distinguish between $2 \beta 2 v$-decay and $2 \beta 0 v$-decay? Let us start with the first problem.

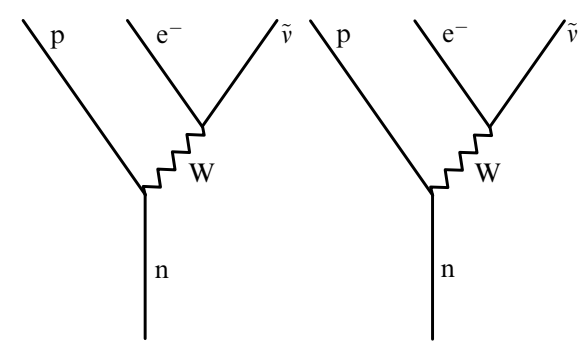

a
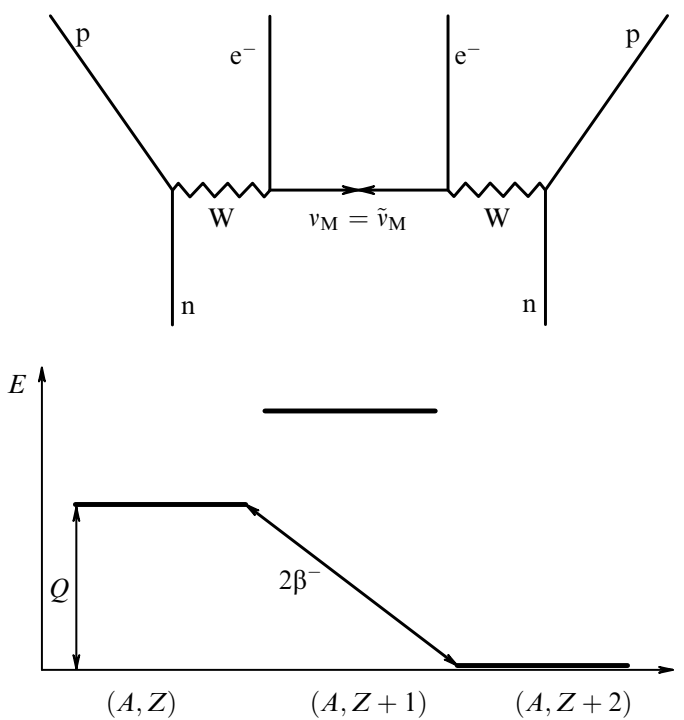

Figure 5. Feynman diagrams for $2 \beta 2 v$-decay (a), $2 \beta 0 v$-decay (b) and the

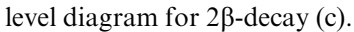

Both $2 \beta 2 v$ - and $2 \beta 0 v$-decay processes can occur if they involve such nuclei $(A, Z)$ for which both $\beta^{-}$- and $\beta^{+}$transitions to adjacent (in charge) nuclei $(A, Z \pm 1)$ are forbidden (either in energy (Fig. 5c) or by selection rules) but double beta transitions to adjacent nuclei with charge variation by two units $(\Delta Z= \pm 2)$ are allowed. Hence the idea of experimental examining double beta decay consists either in attempts to detect 'granddaughter' nuclei $(A, Z \pm 2)$ produced as a result of allowed double beta decay of the parent nuclei $(A, Z)$, for which two successive beta transitions to 'daughter' nuclei,

$$
(A, Z) \rightarrow(A, Z \pm 1) \text { and }(A, Z \pm 1) \rightarrow(A, Z \pm 2),
$$

are forbidden, or in attempts to detect two electrons (positrons) simultaneously emitted by a single nucleus $(A, Z)$.

The answer to the second question (how is one to distinguish between $2 \beta 0 v$-decay and $2 \beta 2 v$-decay?) is equally simple in idea. Indeed, in the first case a single nucleus simultaneously emits two pairs of particles of the same type $\left(2 \mathrm{p}\right.$ and $2 \mathrm{e}^{-}$), i.e. we are dealing with a two-particle process. In the second case three pairs of particles $\left(2 \mathrm{p}, 2 \mathrm{e}^{-}\right.$, and $\left.2 v_{\mathrm{e}}\right)$ are emitted, which is characteristic of a three-particle process. All this implies that in the first case the electron pair must have a strictly defined total energy (the peak $2 \beta 0 v$ in Fig. 6) equal to the threshold energy of the $2 \beta$-transition, while in the second case the energy of the electron pair is characterized by a continuous spectrum extending from zero to the threshold energy (see the curve labelled $2 \beta 2 v$ in Fig. 6).

In accordance with the aforesaid, the search for double beta decay followed two paths. Firstly, the researchers attempted to detect the nuclei of the 'granddaughter' substance, and secondly, they studied the energy spectrum of the electron pairs emitted.

Let us take an example of the first approach. In 1949, Inghram and Reynolds [98] studied tellurium and selenium ores by geochemical methods. Their study made it possible to estimate the amount of substance forming in the double beta decay of ${ }^{130} \mathrm{Te}$ and ${ }^{82} \mathrm{Se}$ :

$$
{ }_{52}^{130} \mathrm{Te} \rightarrow{ }_{54}^{130} \mathrm{Xe} \text { and }{ }_{34}^{82} \mathrm{Se} \rightarrow{ }_{36}^{82} \mathrm{Kr} .
$$

The choice of the appropriate decay schemes in (41) was determined by the chemical inertness of the end products (Xe and $\mathrm{Kr}$ ) facilitating their extraction from the ore samples. Already in 1949 the method produced meaningful results for

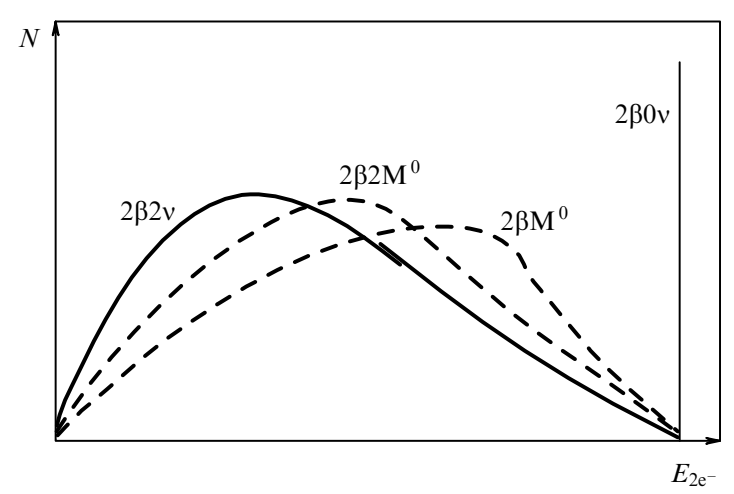

Figure 6. Shape of the double beta spectrum for $2 \beta 2 v-, 2 \beta \mathrm{M}^{0}-, 2 \beta 2 \mathrm{M}^{0}-$, and $2 \beta 0 \mathrm{v}$-decay processes. 
the double beta decay of ${ }^{130} \mathrm{Te}$ [98]:

$$
T_{1 / 2}^{\exp }(2 \beta)_{\mathrm{Te}}=1.4 \times 10^{21} \text { years } .
$$

Much later (in 1992-1993) the following values of $T_{1 / 2}$ were obtained for ${ }^{130} \mathrm{Te}$ and ${ }^{128} \mathrm{Te}[99,100]$ :

$$
\begin{aligned}
& T_{1 / 2}^{\mathrm{exp}}(2 \beta)_{{ }_{130} \mathrm{Te}}=(2.7 \pm 0.1) \times 10^{21} \text { years } \\
& T_{1 / 2}^{\exp }(2 \beta)_{{ }^{128} \mathrm{Te}}=(7.7 \pm 0.4) \times 10^{24} \text { years }
\end{aligned}
$$

Typical values of $T_{1 / 2}$ for the double beta decay of ${ }^{82} \mathrm{Se}$ are $(1-3) \times 10^{20}$ years. By way of an example we give the results of Kirsten et al. [101] and Elliott et al. [102]:

$$
\begin{aligned}
& T_{1 / 2}^{\exp }(2 \beta)_{{ }^{2} \mathrm{Se}}=(1.37 \pm 0.28) \times 10^{20} \text { years } \\
& T_{1 / 2}^{\exp }(2 \beta)_{{ }^{82} \mathrm{Se}}=\left(1.08_{-0.06}^{+0.26}\right) \times 10^{20} \text { years }
\end{aligned}
$$

Of course, by this method it is impossible to distinguish between $2 \beta 2 v$-decay and $2 \beta 0 v$-decay.

The second approach, based on registering electron pairs that a single nucleus emits simultaneously and measuring the electron pair energy, is even more difficult. Since in this case the probability of double beta decay is extremely low, the problem of background noise comes to the fore. To diminish the background effect, the facility must be placed deep underground in a specially constructed laboratory. In addition to shielding the apparatus from cosmic background, certain measures must be taken to lower the radioactive background noise from the detector and surrounding structures.

One can get an idea of just how difficult such experiments may be from the scale of the effect observed. It takes approximately a week to register one event imitating double beta decay. Not a single experiment involving many doublebeta radioactive nuclei has produced a $2 \beta 0 \mathrm{v}$-decay event so far. Practically all experiments of this type have produced or will soon produce a definite value of $T_{1 / 2}$ for $2 \beta 2 \mathrm{v}$-decay, which supports the hypothesis that such a decay mode actually exists. However, for $2 \beta 0 v$-decay all these experiments produce only an estimate of a lower bound on $T_{1 / 2}$, which does not mean that such a decay mode actually exists (although it does not exclude it). Nevertheless, both values carry useful information. The value of $T_{1 / 2}$ in $2 \beta 2 v$-decay is important because it can be compared with results of the Standard Model, which can be used to calculate the matrix elements characterizing the probability of this process. The estimate of $T_{1 / 2}$ for $2 \beta 0 \mathrm{v}$-decay acts as the test mentioned earlier and, in particular, provides an estimate of an upper bound on the Majorana neutrino mass.

In conclusion of this section several remarks concerning the third type of double beta decay, which is being actively discussed by theoreticians, are in order. What we mean are the three-particle schemes of double beta decay in which hypothetical particles known as majorons $\left(\mathrm{M}^{0}\right) \dagger$ come into play:

$$
\begin{aligned}
& (A, Z) \rightarrow(A, Z+2)+2 \mathrm{e}^{-}+\mathrm{M}^{0}, \\
& (A, Z) \rightarrow(A, Z+2)+2 \mathrm{e}^{-}+2 \mathrm{M}^{0} .
\end{aligned}
$$

$\uparrow$ Sometimes these particles are denoted by $\chi$.
As in the case of neutrinoless double beta decay, these decay schemes lie outside the scope of the Standard Model. The majoron involved in these schemes was initially introduced into elementary particle physics as a hypothetical particle weakly interacting with matter and having the following properties: $Z=0, \boldsymbol{\sigma}=0$, and $m=0$ (or extremely small). It was predicted that the particle should appear in models with spontaneous breaking of lepton number conservation. Later the idea of a majoron was extended by including particles with different lepton numbers $(L=0$ and $L=2$ ), Goldstone bosons (massless pseudoscalar particles accompanying spontaneous symmetry breaking), intermediate scalars, intermediate fermions, etc.

Burgess and Cline [103] and Pas et al. [104] discussed nine different models of double beta decay that followed (47) and (48) schemes. A characteristic feature of all these models is the continuous energy spectra of the electron pairs, spectra that differ from each other and the spectrum of $2 \beta 2 v$-decay in the position and height of the peak (see the curves labelled $2 \beta \mathrm{M}^{0}$ and $2 \beta 2 \mathrm{M}^{0}$ in Fig. 6). Comparing these spectra with the experimental spectra of beta decay and taking into account the calculated matrix elements, we can estimate $T_{1 / 2}$ for different decay schemes with majoron emission.

\subsection{Studying double beta decay with the xenon TPC detector}

One of the most effective detectors of double beta decay is the TPC facility in the St. Gotthard Tunnel, Switzerland. The tunnel runs at a depth of 3000-m water equivalent, reducing the muon background by a factor of a million.

The source of double beta decay in the TPC detector is 1801 of xenon enriched to $62.5 \%$ in ${ }^{136} \mathrm{Xe}$ that is kept at a pressure of $5 \mathrm{~atm}$. A distinctive feature of this detector is that it allows recording electron tracks, which facilitates distinguishing double-beta decay events from the background. In particular, because ionization losses grow with decreasing electron velocity, the beginning and end of a track can easily be distinguished. The end of a track exhibits a distinctive bundle ('blob'). Thus, an event connected with double beta decay is associated with two electron tracks that start at a single point and end with two 'blobs'. The energy resolution at an energy corresponding to $2 \beta 0 \mathrm{v}$-decay, $Q=2.48 \mathrm{MeV}$, is found to be $\Delta E / E \simeq 0.066$.

A schematic of the TPC detector is depicted in Fig. 7 [105]. The main elements are an anode system with a recording device and a cathode, with a uniform electrostatic field produced by field-shaper rings in the space between the anode and cathode. To reduce the natural radioactivity background the device is protected by a lead shield $20-$ $30 \mathrm{~cm}$ thick. The shield is surrounded by a plastic bag continuously flushed with nitrogen to remove radon. All this is placed into a copper pressure chamber, with walls $5 \mathrm{~cm}$ thick, for additional shielding.

Events are identified with a special device located behind the anode plane; the device automatically writes the projections $X$ and $Y$ of the track coordinates. Coordinate $Z$ is found by measuring the time of drift of the ionization electrons along the chamber. Hence the abbreviation TPC (Time Projection Chamber).

The device described and its variant modernized in 1992 were operational for 13357 hours. The resulting spectrum of double-beta decay events is depicted in Fig. 8. Clearly, the expected peak at $E_{2 \mathrm{e}}=2.48 \mathrm{MeV}$ from the $2 \beta 0 \mathrm{v}$-decay is not present. Processing the results with allowance made for 


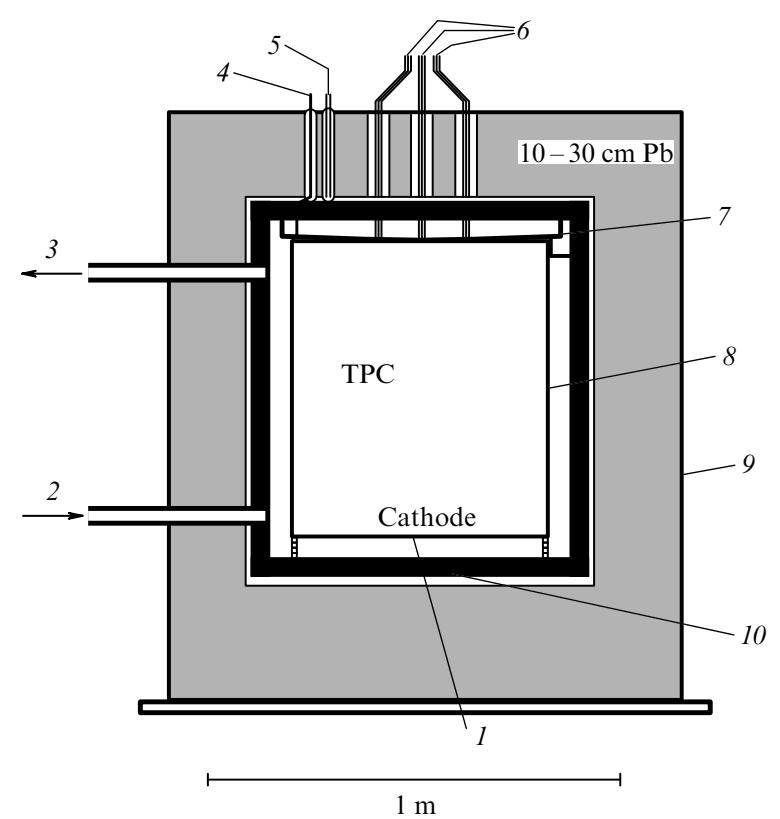

Figure 7. Schematic of the TPC device: 1 - cathode, 2 - gas inlet, 3 - gas outlet, 4 - grid, 5 - anode, 6 - preamplifiers, 7 - a new anode system, 8 - field-shaper rings, 9 - neutron shielding, and 10 - copper chamber.

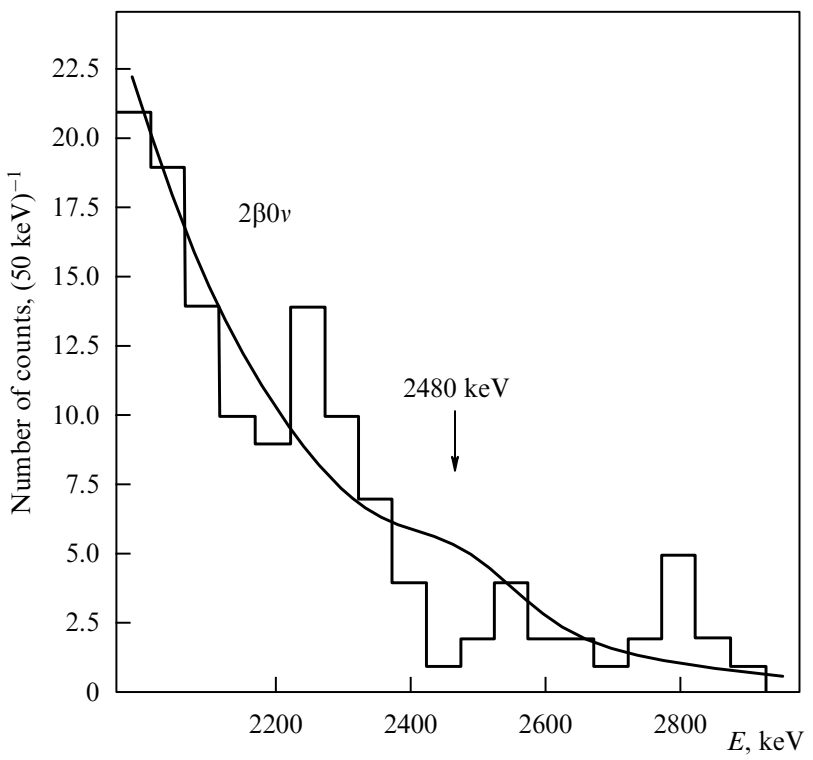

Figure 8. Energy spectrum of two-electron $2 \beta 0 v$-type events measured with the TPC device.

background noise yields the following estimate:

$$
T_{1 / 2}(2 \beta 0 v) \geqslant 4.2 \times 10^{23} \text { years }(90 \% \text { c.l. }) \text {. }
$$

By employing the results of the calculations of matrix elements done by Engel et al. [106] and Staudt et al. [107] one can arrive at the following estimates for the upper bound on the Majorana neutrino mass: $m_{v} \leqslant 2.4-2.8 \mathrm{eV}$ [106], and $m_{v} \leqslant 2.4 \mathrm{eV}$ [107]. The new data of 1994-1995 yield an estimate of $T_{1 / 2}$ for $2 \beta 2 \mathrm{v}$-decay, namely

$$
T_{1 / 2}(2 \beta 2 v) \geqslant 5.6 \times 10^{20} \text { years }(90 \% \text { c.l. })
$$

and an estimate for double beta decay with emission of a majoron:

$$
T_{1 / 2}^{\mathrm{M}^{0}} \geqslant(1.1-1.5) \times 10^{22} \text { years }(90 \% \text { c.l. }) .
$$

The result presented in (50) for $T_{1 / 2}(2 \beta 2 \mathrm{v}$-decay) is close to the value $(8-20) \times 10^{20}$ years for ${ }^{136} \mathrm{Xe}$ predicted by Engel et al. [106] but differs considerably from the value $T_{1 / 2}(2 \beta 2 v)=(14-211) \times 10^{20}$ years predicted by Staudt et al. [107]. The authors of the report [105] under discussion state that their immediate goal is to observe directly the $2 \beta 2 v$-mode of the double beta decay of ${ }^{136} \mathrm{Xe}$.

\subsection{Studying the double beta decay of ${ }^{100} \mathrm{Mo}$ and ${ }^{116} \mathrm{Cd}$ with the NEMO detector}

Hubert [108] describes a detector built as a result of the NEMO (Neutrino Experiment with Molybdenum) Collaboration, which, like the TPC detector, makes it possible to detect directly the electrons emitted in the course of double beta decay. The distinctive features of the NEMO facility are: mutual independence of the source and detector, which enables studying different $2 \beta$-sources; the possibility of obtaining the full characteristics of two-electron decay processes, including the reconstruction of electron paths and measurements of the energy and flight time; the possibility of extrapolating to large source sizes, and the high stability of the device's operation in time and the low level of background noise produced by the device.

In all there are three prototypes of the NEMO facility. The first prototype, NEMO-1, had no source of double beta decay and was used only to record one-electron tracks with an energy higher than $100 \mathrm{keV}$ by a system of Geiger counters and plastic scintillators. In addition to the detecting part, the second prototype, NEMO-2 (Fig. 9a) contained a source of double beta decay, which was a plate measuring $1 \mathrm{~m} \times 1 \mathrm{~m} \times 40 \mu \mathrm{m}$ that divided the detector's volume into two parts. One side of the plate contained $172 \mathrm{~g}$ of molybdenum enriched to $98.4 \%$ in ${ }^{100} \mathrm{Mo}$ (the layer of molybdenum was $40 \mu \mathrm{m}$ thick), and the other contained 163 $\mathrm{g}$ of natural molybdenum $\left(9.6 \%\right.$ in $\left.{ }^{100} \mathrm{Mo}\right)$ (this layer was $44 \mu \mathrm{m}$ thick). Each of the two parts of the detector's volume consisted of 10 layers of Geiger counters, with 32 counters in each layer (oriented vertically and horizontally). The energy and the flight time were measured by two external layers of plastic counters. To reduce multiple scattering, the track volume was filled with helium at a pressure of $1 \mathrm{~atm}$ with a $4 \%$ addition of alcohol.

NEMO-2 (as NEMO-1) was positioned in an underground laboratory in Frejus, France, and it was used for a thorough study of the background and for estimating $T_{1 / 2}(2 \beta 2 v)$ and $T_{1 / 2}(2 \beta 0 v)$ relevant to ${ }^{100} \mathrm{Mo}$ :

$$
\begin{aligned}
& T_{1 / 2}(2 \beta 2 v)=\left(0.95 \pm 0.04_{\text {stat }} \pm 0.09_{\text {syst }}\right) \times 10^{19} \text { years } \\
& T_{1 / 2}(2 \beta 0 v) \geqslant 6.4 \times 10^{21} \text { years }(90 \% \text { c.l. })
\end{aligned}
$$

In the second experiment conducted using NEMO-2, the source was a central foil with a surface area of $1 \mathrm{~m}^{2}$. One side of the foil contained $152 \mathrm{~g}$ of cadmium enriched to $93.2 \%$ in ${ }^{116} \mathrm{Cd}$, while the other contained $143 \mathrm{~g}$ of natural cadmium $\left(7.58 \%\right.$ in $\left.{ }^{116} \mathrm{Cd}\right)$. After the background was subtracted, it was found that for ${ }^{116} \mathrm{Cd}$

$$
T_{1 / 2}(2 \beta 2 v)=\left(3.4 \pm 0.4_{\text {stat }} \pm 0.3_{\text {syst }}\right) \times 10^{19} \text { years } .
$$



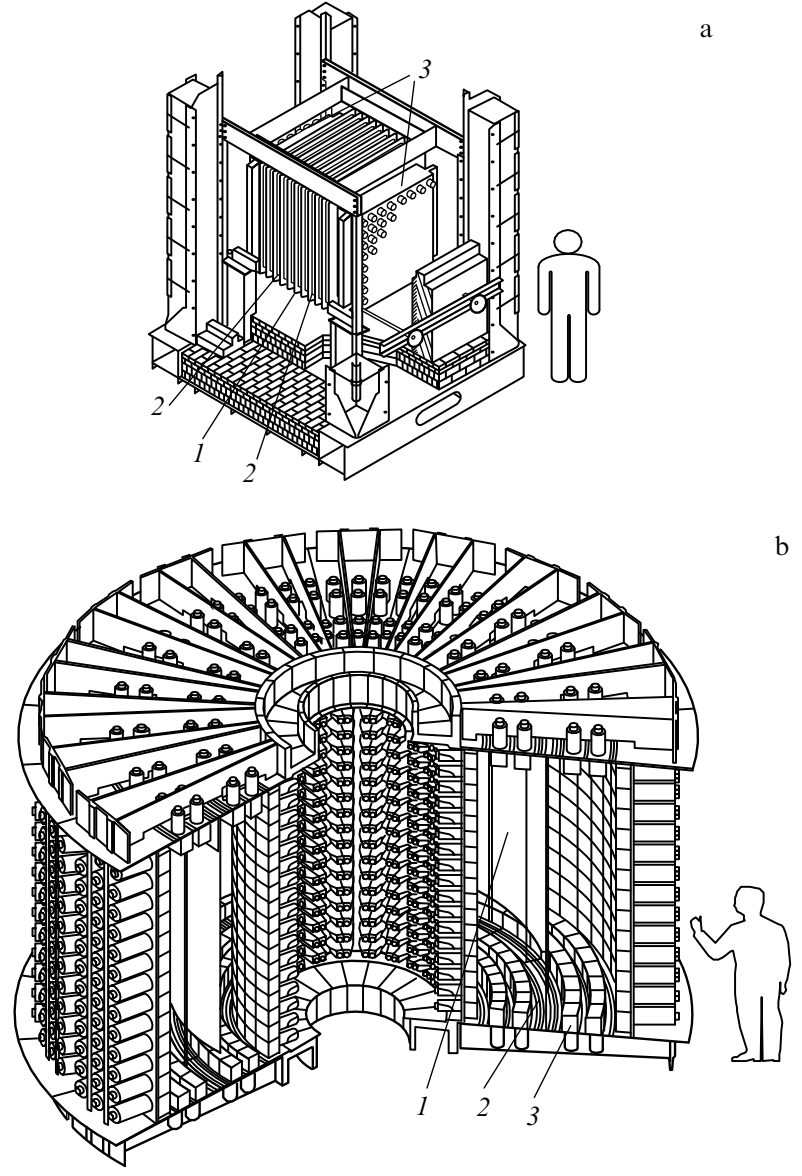

Figure 9. The NEMO-2 (a) and NEMO-3 (b) detectors: (a) 1 - the central frame with a metal foil, 2 - the track unit consisting of 10 frames with $2 \times 32$ Geiger counters in each, 3 - the scintillation device consisting of $8 \times 8$ counters; (b) 1 - the foil acting as the source, 2 - track detectors, 3 - scintillation devices.

The researchers notice that the values obtained for the matrix elements estimated by the measured values of $T_{1 / 2}$ for ${ }^{100} \mathrm{Mo}$ and ${ }^{116} \mathrm{Cd}$ practically coincide with the estimates obtained in other experiments and do not contradict the results of theoretical approaches.

In 1995, the NEMO Collaboration started the construction of the third variant of a detector, NEMO-3 (Fig. 9b). The detector is cylindrical in shape with a central thin foil $2 \mathrm{~m}$ high and $2.8 \mathrm{~m}$ in diameter placed in between two concentric volumes of track detectors. To identify electron-positron pairs which were produced by the high-energy gamma quanta generated in neutron capture, a $30 \mathrm{G}$ magnetic field will be used in the device.

These first experiments involving the NEMO-3 detector are planned for 1998. The source of double beta decay will be $10 \mathrm{~kg}$ of molybdenum enriched in ${ }^{100} \mathrm{Mo}$, although the researchers do not exclude the possibility of using other sources such as ${ }^{82} \mathrm{Se},{ }^{96} \mathrm{Zr},{ }^{116} \mathrm{Cd}$, etc.

\subsection{Studying double beta decay with} a germanium detector enriched in ${ }^{76} \mathrm{Ge}$

Today the most sensitive experiment in the study of double beta decay is that of the Heidelberg-Moscow Collaboration, an experiment that uses the $2 \beta$-decay source with ${ }^{76} \mathrm{Ge}$ enriched to $86 \%$. Klapdor-Kleingrothaus [109] and Balysh et al. [110] obtained the most precise value of $T_{1 / 2}(2 \beta 2 v)$ and an upper bound on $T_{1 / 2}(2 \beta 0 \mathrm{v})$, which leads to a lower bound on the Majorana neutrino mass $\left(m_{v}<0.6 \mathrm{eV}\right)$.

A remarkable feature of the detector manufactured from ${ }^{76} \mathrm{Ge}$ (and of the TPC detector manufactured from ${ }^{136} \mathrm{Xe}$ ) is that it combines two functions, that of the emitter and of a registering device, while in contrast to ${ }^{136} \mathrm{Xe}$ the detection process that uses ${ }^{76} \mathrm{Ge}$ has an exceptionally high resolution power $\Delta E / E \simeq 3 \times 10^{-3}$ and practically $100 \%$ efficiency. The $2 \beta 0 \mathrm{v}$-decay of ${ }^{76} \mathrm{Ge}$ produces a monochromatic line at $2038.58 \pm 0.31 \mathrm{keV}$ in the energy spectrum for an electron pair.

The Heidelberg-Moscow Collaboration had $19.2 \mathrm{~kg}$ of germanium enriched in ${ }^{76} \mathrm{Ge}$, which was used to manufacture five high-purity p-type semiconductor detectors with a total mass of $11.5 \mathrm{~kg}$. At present all five detectors are operational in the underground laboratory near Gran Sasso, Italy at a depth of $3500-\mathrm{m}$ water equivalent. A detailed description of the facility is given in Refs $[111-115]$. Figure $10 \mathrm{a}$, b shows the integral spectrum containing all the data for detectors with a statistical significance of $12 \mathrm{~kg} \times \mathrm{y}$ except for the first 200 days of operation (to exclude the effect of short-lived impurities). Figure 10c depicts the part of the spectrum in the vicinity of the hypothetical peak corresponding to the $2 \beta 0 \mathrm{v}$-decay of

${ }^{76} \mathrm{Ge}$. Clearly, there is no signal from $2 \beta 0 v$-decay, which yields the following estimate for a lower bound on the half-life for this process:

$$
T_{1 / 2}^{0 v}\left({ }^{76} \mathrm{Ge}\right)>6.4(10.0) \times 10^{24} \text { years }
$$

within $90 \%(68 \%)$ confidence level. The lower bound obtained for $T_{1 / 2}(2 \beta 0 \mathrm{v})$ and the value of the matrix element calculated by Staudt et al. [107] were used in estimating an upper bound on the effective Majorana electron neutrino mass (with weak right-handed currents ignored):

$$
\begin{aligned}
& \left\langle m_{v}\right\rangle \leqslant 0.6 \mathrm{eV} \quad(90 \% \text { c.l. }), \\
& \left\langle m_{v}\right\rangle \leqslant 0.5 \mathrm{eV}(68 \% \text { c.l. }) .
\end{aligned}
$$

Moreover, following Muto et al. [116] and the value obtained for $T_{1 / 2}(2 \beta 0 \mathrm{v})$, the researchers arrived at an estimate of a lower bound on the mass of the left-handed superheavy neutrino,

$$
\left\langle m_{v_{\mathrm{H}}}\right\rangle \geqslant 5.5 \times 10^{7} \mathrm{GeV},
$$

and, using the results of Pas et al. [104], the researchers arrived at an estimate for a lower bound on the mass of the righthanded W-boson,

$$
m_{\mathrm{W}_{R}} \geqslant 1 \mathrm{TeV} \text {. }
$$

While at present the search for $2 \beta 0 v$-decay leads only to a lower bound on $T_{1 / 2}$ and estimates of other quantities that follow from it, for $T_{1 / 2}$ of the $2 \beta 2 v$-decay of ${ }^{76} \mathrm{Ge}$ this experiment made it possible to obtain a fairly exact value

$$
T_{1 / 2}(2 \beta 2 v)=\left(1.7_{-0.11}^{+0.13}\right) \times 10^{21} \text { years. }
$$

In addition to the above results, which refer to $2 \beta 0 \mathrm{v}$ - and $2 \beta 2 v$-decay schemes, the measurements of Klapdor-Kleingrothaus [109] were used to calculate the expected values of $T_{1 / 2}$ for the two schemes (47) and (48) with different majoron types. The calculated values were found to lie in the range $5.85 \times 10^{21}$ to $7.91 \times 10^{21}$ years. In the next five years, the Heidelberg-Moscow Collaboration plans to move into the sub-electron-volt mass range down to $0.1 \mathrm{eV}$. 

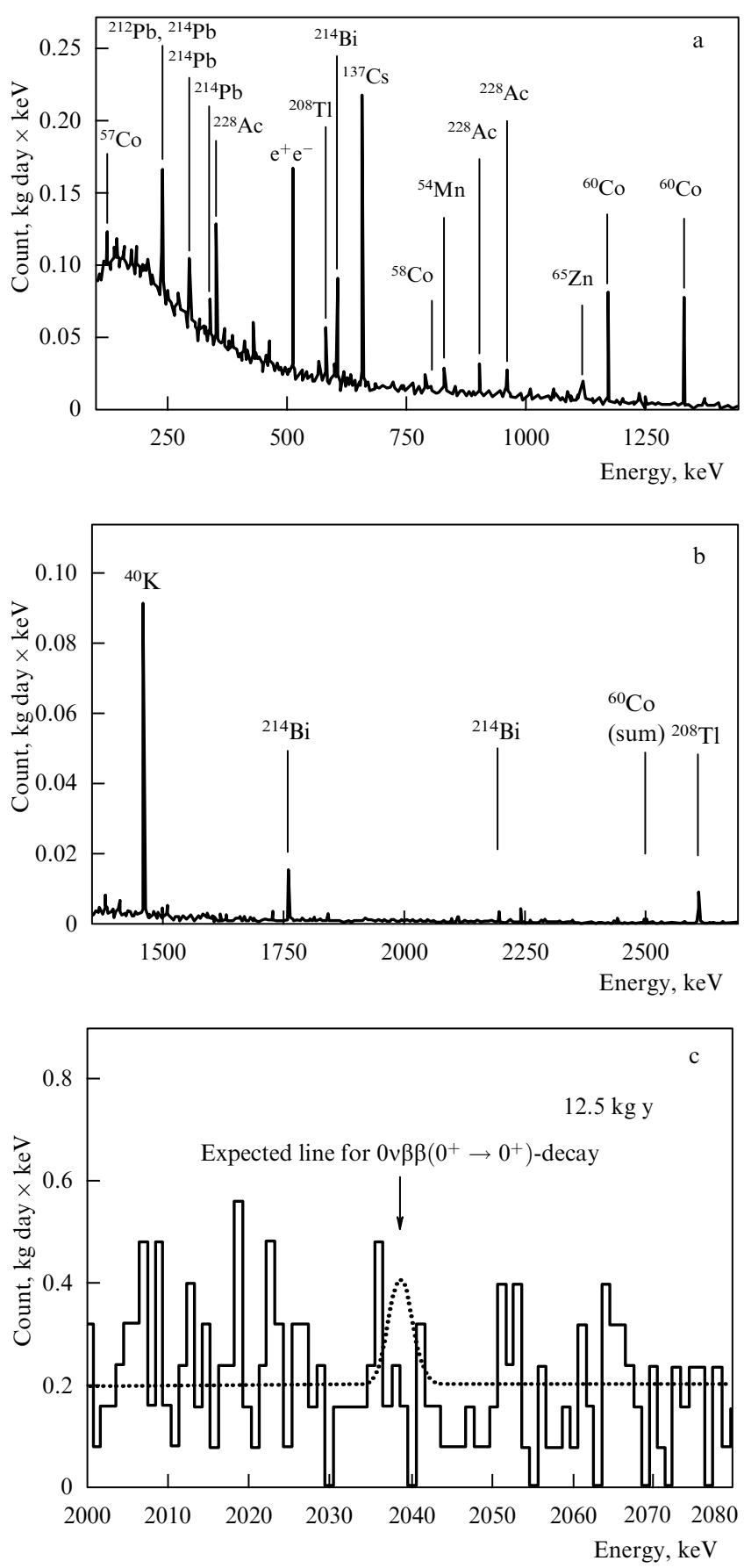

Figure 10. Two-electron spectra recorded by the ${ }^{76} \mathrm{Ge}$ detector: (a) and (b) - integral spectra, and (c) - the part of the spectrum relating to the expected $2 \beta 0 v$-decay process.

\subsection{Summary of the results of $2 \beta$-decay research}

Figure 11a, which was taken from Klapdor-Kleingrothaus's report [109], shows the best results obtained so far in doublebeta decay studies, with estimates of the half-life exceeding $10^{21}$ years. In addition to the record-breaking data in Klapdor-Kleingrothaus's report [109] that involved ${ }^{76} \mathrm{Ge}$, we also list the results for double beta decay of ${ }^{48} \mathrm{Ca}[117],{ }^{82} \mathrm{Se}$ [118], ${ }^{100} \mathrm{Mo}[119],{ }^{116} \mathrm{Cd}$ [120], ${ }^{130} \mathrm{Te}$ [121], ${ }^{136} \mathrm{Xe}[122],{ }^{150} \mathrm{Nd}$ [123], ${ }^{100} \mathrm{Mo}[124],{ }^{48} \mathrm{Ca}[125]$, and ${ }^{136} \mathrm{Xe}$ [11]. Figure $11 \mathrm{~b}$ depicts the estimates of $1 / m_{v}$ obtained from the data shown in Fig. 11a.
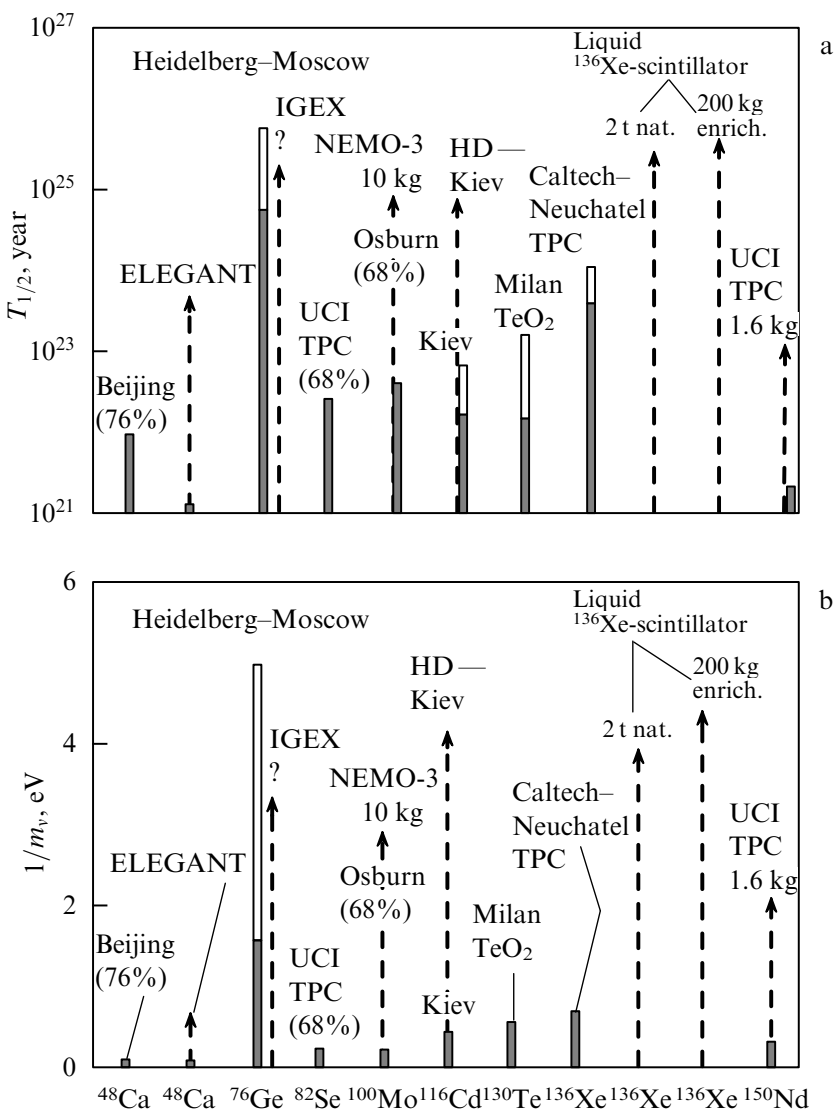

Figure 11. Main results of determination of $T_{1 / 2}(2 \beta 0 v)$ and $1 / m_{v}$ obtained by 1995 (white) and forecast for the year 2000 (shaded): (a) the limits for $T_{1 / 2}(2 \beta 0 v)$, and (b) the limits for $1 / m_{v}$.

\section{Neutrino oscillations}

In 1957-1958, Bruno Pontecorvo [126, 127] hypothesized the possibility of neutrino oscillations. On the one hand, at that time it was known that there exist oscillations of neutral Kmesons (such oscillations were discovered in 1955), which proceed with violation of the law of strangeness conservation $(\Delta S=2)$, and, on the other hand, the hypothesis that the law of lepton number conservation could be violated $(\Delta L=2)$ and the possibility of the neutrino mass being finite were actively being discussed.

The analogy between these two conservation-law violations, with each violation relating a particle and an antiparticle $\left(\mathrm{K}^{0} \leftrightarrow \widetilde{\mathrm{K}}^{0}, v \leftrightarrow \tilde{\mathrm{v}}\right)$, led Pontecorvo to the initial scheme of $v$-oscillations, $v_{\mathrm{e}} \leftrightarrow \tilde{v}_{\mathrm{e}}^{L}$, where $\tilde{v}_{\mathrm{e}}^{L}$ is the left-handed (sterile under the $(\mathrm{V}-\mathrm{A})$-interaction) antineutrino.

After discovering the muon neutrino $v_{\mu}$ in 1962, Maki et al. [128] studied another neutrino-oscillation scheme, the one in which the neutrinos changed their type (flavour), $v_{\mathrm{e}} \leftrightarrow v_{\mu}$. In this scheme the lepton-number conservation law is violated in the sense that $\Delta L_{\mathrm{e}}=1$ and $\Delta L_{\mu}=1$. Independently of Maki et al. [128], the idea of two different types of neutrino being able to mix was examined in detail in 1967 by Pontecorvo [129] and then by Gribov and Pontecorvo [130] in 1969. They drew in particular attention to the fact that the oscillations of solar neutrinos could explain the neutrino deficit observed by Davis et al. [131]. In the years that followed the interest in neutrino oscillations steadily grew in 
connection with active discussions concerning the possibility that the neutrino mass might be finite.

After discovering the tau lepton in 1975-1978 and the intensive studies of the various decay schemes for this particle, the possibility of a third type of neutrino, $v_{\tau}$, became quite real. Such a neutrino would also participate in the oscillation process within the following schemes: $v_{\mathrm{e}} \leftrightarrow v_{\tau}$ and $v_{\mu} \leftrightarrow v_{\tau}$. The mixing of neutrinos of all three types was discussed in detail by Bilen'kiı̌ [132] in 1987.

\subsection{Neutrino mixing and oscillation hypotheses}

Let us now briefly discuss the hypothesis on neutrino mixing, which if valid must lead to neutrino oscillations. According to this hypothesis, in addition to the well-known physical neutrinos $v_{\mathrm{e}}, v_{\mu}$, and $v_{\tau}$, which are eigenstates of the weakinteraction Hamiltonian, there exist also three types of neutrino, $v_{1}, v_{2}$, and $v_{3}$ being the eigenstates of mass. The Hamiltonian states characterize the weak processes of neutrino creation and absorption, proceeding with the lepton numbers conservation. They do not have definite values of mass (compare this with $\mathrm{K}^{0}$ - and $\widetilde{\mathrm{K}}^{0}$-mesons participating in creation and annihilation processes with strangeness conservation). The other eigenstates have definite values of mass and, hence, characterize neutrino movement in space-time (compare this with $\mathrm{K}_{S^{-}}^{0}$ and $\mathrm{K}_{L}^{0}$-mesons). Here, just as the pair of wave functions of $\left(\mathrm{K}^{0}-\mathrm{K}^{0}\right)$-mesons can be expressed in terms of the pair of wave functions of the $\left(\mathrm{K}_{S}^{0}-\mathrm{K}_{L}^{0}\right)$-mesons and vice versa, the triple of physical neutrinos $v_{e}, v_{\mu}$, and $v_{\tau}$ can be expressed in terms of the triple of neutrinos with definite masses (and vice versa). One can imagine any physical neutrino $\left(v_{\mathrm{e}}, v_{\mu}\right.$ and $\left.v_{\tau}\right)$ as being a kind of 'mixture' of three neutrinos with definite masses $\left(v_{1}\right.$, $v_{2}$, and $v_{3}$ ), and therefore a physical neutrino has no definite mass. We will see that if the masses of $v_{1}, v_{2}$, and $v_{3}$ are different, the mixing must lead to transformations of $v_{\mathrm{e}}$ into $v_{\mu}$ and $v_{\tau}$ and similar processes, i.e. oscillations.

We notice again that in contrast to the real mixing of $\mathrm{K}^{0}$ mesons, neutrino mixing is plausible, but is still a hypothesis, yet to be proved in experiments. The discovery of neutrino oscillations, if it ever happens, will be proof of validity of this hypothesis.

\subsection{Mathematical formalism of neutrino oscillations}

Let us assume that each of the three neutrinos $v_{1}, v_{2}$, and $v_{3}$ has a definite mass, $m_{1}, m_{2}$, and $m_{3}$, and examine the mathematics of the mixing process leading to neutrino oscillations. Bearing in mind the complexity of describing the general mixing process involving the three types of neutrinos (see [132]), we consider only the simple case of oscillations involving physical neutrinos of two types, say $v_{\mathrm{e}}$ and $v_{\mu}$ (other pairs, $v_{e}, v_{\tau}$ and $v_{\mu}, v_{\tau}$, could equally be used) and two types of neutrinos with definite masses, say $v_{1}$ and $v_{2}$.

In this case the transformation coefficients are reduced to a 2-by-2 Cabibbo type matrix

$$
\left|\begin{array}{cc}
\cos \theta & \sin \theta \\
-\sin \theta & \cos \theta
\end{array}\right|
$$

with a single parameter, the mixing angle. Using this matrix, we can express the wave functions of the physical neutrinos $v_{\mathrm{e}}$ and $v_{\mu}$ in terms of the wave functions of $v_{1}$ and $v_{2}$ as follows:

$$
\begin{aligned}
& \psi_{v_{\mathrm{e}}}=\psi_{v_{1}} \cos \theta+\psi_{v_{2}} \sin \theta, \\
& \psi_{v_{\mu}}=-\psi_{v_{1}} \sin \theta+\psi_{v_{2}} \cos \theta .
\end{aligned}
$$

We assume, for clarity sake, that the mixing angle $\theta$ determines the 'fraction' of the states $v_{1}$ and $v_{2}$ in $v_{\mathrm{e}}$ and $v_{\mu}$. At $\theta=0^{\circ}, \psi_{v_{\mathrm{e}}}=\psi_{v_{1}}$ and $\psi_{v_{\mu}}=\psi_{v_{2}}$, i.e. the laws of lepton number conservation are strictly obeyed and there is no mixing, with $v_{\mathrm{e}}$ and $v_{\mu}$ having definite masses equal to $m_{1}$ and $m_{2}$, respectively. At $\theta=45^{\circ}$, the mixing is at its maximum, i.e. states $v_{1}$ and $v_{2}$ present to the same extent both in $v_{\mathrm{e}}$ and $v_{\mu}$. The states $v_{e}$ and $v_{\mu}$ have definite lepton numbers but their masses are undefined.

If the formulae (62) are reversed, they become

$$
\begin{aligned}
& \psi_{v_{1}}=\psi_{v_{\mathrm{e}}} \cos \theta-\psi_{v_{\mu}} \sin \theta, \\
& \psi_{v_{2}}=\psi_{v_{\mathrm{e}}} \sin \theta+\psi_{v_{\mu}} \cos \theta,
\end{aligned}
$$

and describe neutrino states with definite masses but undefined lepton numbers.

Now let us examine the 'dynamics' of mixing in time. Suppose that initially, at $t=0$, only the state $v_{\mathrm{e}}$ is formed. Then $\psi_{v_{\mathrm{e}}}(0)=1$ and $\psi_{v_{\mu}}(0)=0$. According to (62), at time $t$ we have

$$
\psi_{v_{\mathrm{e}}}(t)=\psi_{v_{1}}(t) \cos \theta+\psi_{v_{2}}(t) \sin \theta,
$$

where $\psi_{v_{1}}(t)$ and $\psi_{v_{2}}(t)$, being states with definite masses, vary in time according to the following equations

$$
\begin{aligned}
& \psi_{v_{1}}(t)=\psi_{v_{1}}(0) \exp \left(-\mathrm{i} E_{1} t\right), \\
& \psi_{v_{2}}(t)=\psi_{v_{2}}(0) \exp \left(-\mathrm{i} E_{2} t\right),
\end{aligned}
$$

where we assume, provided that $m_{1,2} \ll p$, that $E_{1,2}$ equals $(\hbar=c=1)$

$$
E_{1,2}=p+\frac{m_{1,2}^{2}}{2 p} .
$$

If we then substitute (63) and (65) into (64) and allow for the fact that $\psi_{v_{\mathrm{e}}}(0)=1$, we arrive at

$$
\psi_{v_{\mathrm{e}}}(t)=\cos ^{2} \theta \exp \left(-\mathrm{i} E_{1} t\right)+\sin ^{2} \theta \exp \left(-\mathrm{i} E_{2} t\right),
$$

which after fairly simple transformations yields

$$
\left|\psi_{v_{\mathrm{e}}}(t)\right|^{2}=1-\sin ^{2} 2 \theta \sin ^{2} \frac{\left(E_{2}-E_{1}\right) t}{2} .
$$

This expression gives the probability $P_{v_{\mathrm{e}}, v_{\mathrm{e}}}(t)$ that a neutrino which at time $t=0$ was a purely electron neutrino $\left(v_{\mathrm{e}}\right)$ remains so at time $t$, i.e. the probability of $v_{\mathrm{e}}$ nonvanishing because of oscillations (the probability of $v_{\mathrm{e}}$ 'survival'). If in accordance with (66) we replace $E_{2}-E_{1}$ by $\left(m_{2}^{2}-m_{1}^{2}\right) / 2 p=\Delta m^{2} c / 2 E$ and the time of flight $t$ by the distance to the source $R=c t$, we obtain

$$
P_{v_{\mathrm{e}}, v_{\mathrm{e}}}(t)=1-\sin ^{2} 2 \theta \sin ^{2} \frac{\Delta m^{2} R}{4 E},
$$

or

$$
P_{v_{\mathrm{e}}, v_{\mathrm{e}}}(t)=1-\sin ^{2} 2 \theta \sin ^{2} \frac{\pi R}{L},
$$

where

$$
L=\frac{4 \pi E}{\Delta m^{2}}
$$

is the oscillation length 
By subtracting $P_{v_{\mathrm{e}}, v_{\mathrm{e}}}(t)$ from unity we get the probability $P_{v_{e}, v_{\mu}}(t)$ of the disappearance of $v_{\mathrm{e}}$ due to oscillations, i.e. the probability of $v_{\mathrm{e}}$ becoming $v_{\mu}$ after the neutrino has travelled the distance $R$ :

$$
P_{v_{\mathrm{e}}, v_{\mu}}(t)=1-P_{v_{\mathrm{e}}, v_{\mathrm{e}}}(t)=\sin ^{2} 2 \theta \sin ^{2} \frac{\pi R}{L} .
$$

From (70) and (72) we see that the oscillation length $L$ determines the alteration of the maxima and minima in $P_{v_{e}, v_{e}}$ and $P_{v_{e}, v_{\mu}}$ as a function of the distance to the source. The first maximum in the probability $P_{v_{\mathrm{e}}, v_{\mu}}(R)$ of $v_{\mu}$ appearing instead of the vanished $v_{\mathrm{e}}$ can be expected at a distance $R=L / 2$, and the first maximum in the probability of $v_{\mathrm{e}}$ 'regeneration' can be expected at $R=L$. There is no sense in using the subsequent maxima (at $R=3 L / 2,2 L$, etc.) because of the decrease in beam intensity (for isotropic sources) with increasing distance. When $R \gg L$ or $R \ll L$, the oscillations cannot be seen due to the averaging (vanishing) of $\sin ^{2}(\pi R / L)$.

Thus, the oscillation length $L$ determines the optimum distance $R$ at which one should expect an oscillation effect to occur over the range $\Delta m^{2}=4 \pi E / L$. To change the range within which oscillations can occur, $\Delta m^{2}$, one should change $E / L$, i.e. $E / R$. We notice also that the expression $L=4 \pi E / \Delta m^{2}$ clearly shows that $m_{1} \neq m_{2} \neq 0$ for all possible oscillations. There are no oscillations $(L=\infty)$ if $\Delta m^{2}=0$, i.e. either for $m_{1}=m_{2} \neq 0$ or $m_{1}=m_{2}=0$.

\subsection{General scheme of processing} experimental data

Equations (70) and (72) are pictorial but not very convenient for practical calculations in processing experimental data. Here different formulae are employed, namely

$$
\begin{aligned}
& P_{v_{\mathrm{e}}, v_{\mathrm{e}}}(R, E)=1-\sin ^{2} 2 \theta \sin ^{2} 1.27 \frac{\Delta m^{2} R}{E}, \\
& P_{v_{\mathrm{e}}, v_{\mu}}(R, E)=\sin ^{2} 2 \theta \sin ^{2} 1.27 \frac{\Delta m^{2} R}{E},
\end{aligned}
$$

in which $\left[\Delta m^{2}\right]=\mathrm{eV}^{2},[R]=\mathrm{m}$, and $[E]=\mathrm{MeV}$ (or $[R]=\mathrm{km}$ and $[E]=\mathrm{GeV})$. In these units the oscillation length is

$$
L=\frac{\pi E}{1.27 \Delta m^{2}} \simeq 2.5 \frac{E}{\Delta m^{2}} .
$$

Equations (73) and (74) show that the effect of the disappearance of $v_{\mathrm{e}}$ (and the emergence of $v_{\mu}$ ) caused by oscillations can be detected if $\sin ^{2} 2 \theta \sin ^{2}\left(1.27 \Delta m^{2} R / E\right)$ considerably differs from zero. In particular, if the experimental error is approximately $25 \%$, the condition is met when $\sin ^{2} 2 \theta=1$, or $\theta=45^{\circ}$ (total mixing), and the second factor is $\sin ^{2}\left(1.27 \Delta m^{2} R / E\right)=0.25$ i.e. $1.27 \Delta m^{2} R / E \simeq 0.5 \quad\left(30^{\circ}\right)$, which yields $\Delta m^{2} R \simeq 0.4 E / R$ for the boundary value, where $R$ is the maximum possible distance from the source at which measurements with the given error can be conducted.

Suppose, for instance, the source of neutrinos is a reactor $\left(\bar{E}_{\mathrm{v}} \simeq 4 \mathrm{MeV}\right)$ with an intensity so high that one can conduct measurements at a distance $R \simeq 100 \mathrm{~m}$. Then for the minimum boundary value we get $\Delta m^{2}=0.4(4 / 100)=$ $1.6 \times 10^{-2} \mathrm{eV}^{2}$

If the mixing is not total $\left(\sin ^{2} 2 \theta<1\right)$, it is still possible to observe oscillations at the same distances $R$, but $\Delta m^{2}$ must be higher. For instance, let us take the 'symmetric' case, where $\sin ^{2} 2 \theta=0.25$ and $\sin ^{2}\left(1.27 \Delta m^{2} R / E\right)=1$. We then find that at a distance of a hundred meters $\Delta m^{2}=5 \times 10^{-2} \mathrm{eV}^{2}$.

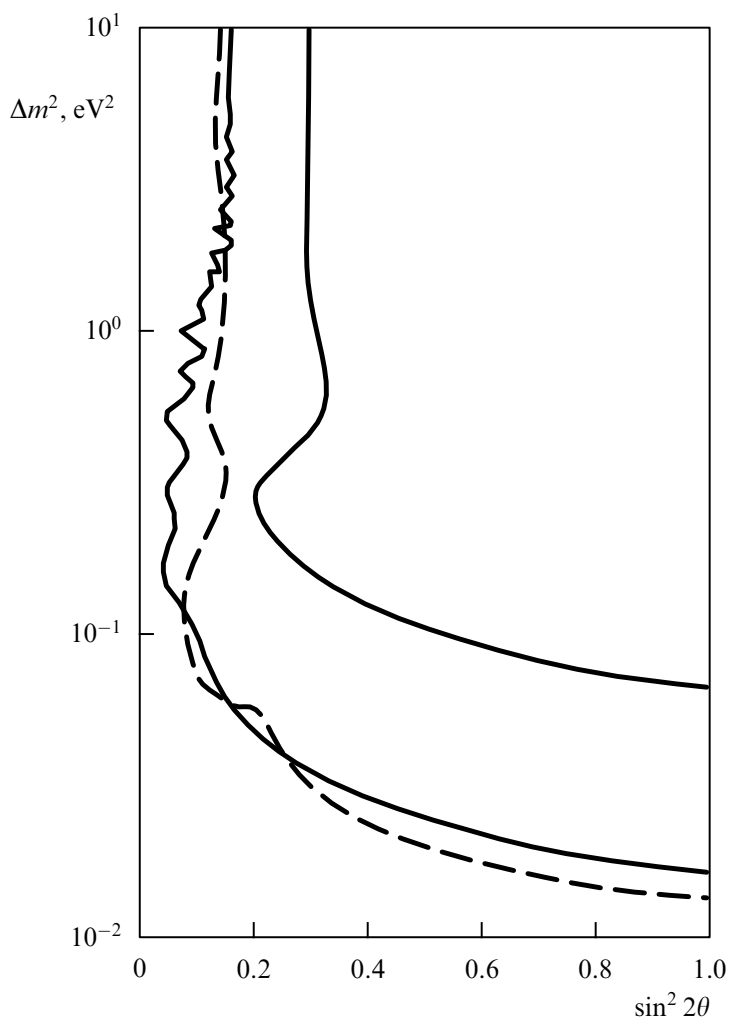

Figure 12. Typical curves in neutrino oscillation experiments.

Thus, the curves that limit the region where oscillations can exist are shaped as in Fig. 12, taken from the paper of Vidyakin et al. [133] that reports on research done in Krasnoyarsk. Above the curves and to the right is the region where there can be no oscillations (this has been verified by experiments). Below the curves and to the left is the region where the search for oscillations can be continued.

Equations (73) and (74) describe the probabilities of the disappearance of $v_{\mathrm{e}}$. Hence it is tacitly assumed that we must compare the measured value of the intensity of the neutrino beam at a given distance $R$ from the reactor with the calculated value that allows for a decrease in intensity with increasing distance (without allowing for oscillations).

But if it is possible to measure the intensity of the neutrino beam at different distances from the source, another approach can be used, i.e. we can compare the measured beam's intensity ratio at these distances with the calculated ratio. The advantage of this approach is that the results are practically independent of the shape of the neutrino spectrum.

\subsection{Search for solar neutrino oscillations.}

The deficit in $v_{\mathrm{e}}$ and the Mikheev-Smirnov-Wolfenstein effect. The hypothesis of the existence of a neutrino magnetic moment

It was perhaps solar neutrinos (and, as will shortly see, reactor antineutrinos) that entered the story of the search for neutrino oscillations in the most dramatic way. We would like to mention the extraordinary efforts that were made to discover the solar neutrino deficit and possibly the most promising resonance effect, which became known as the MikheevSmirnov-Wolfenstein effect. Also worth mentioning is the hypothesis that the neutrino has a magnetic moment, which can interact with the magnetic field of the Sun. 
5.4.1 Neutrino model of the Sun. The reader will recall that according to the commonly accepted view on the nature of solar energy, thermonuclear reactions are the source of this energy, which have become known as proton-proton and carbon-nitrogen-oxygen (CNO) cycles.

The proton-proton cycle essentially consists of three reactions:

$$
\begin{aligned}
& 2(\mathrm{p}+\mathrm{p}) \rightarrow 2\left(\mathrm{~d}+\mathrm{e}^{+}+v\right), \\
& 2(\mathrm{~d}+\mathrm{p}) \rightarrow 2\left({ }^{3} \mathrm{He}+\gamma\right), \\
& { }^{3} \mathrm{He}+{ }^{3} \mathrm{He} \rightarrow{ }^{4} \mathrm{He}+2 \mathrm{p} .
\end{aligned}
$$

The energy release in this chain of reactions is $Q=26 \mathrm{MeV}$, and the neutrinos created in this chain carry an energy $\leqslant 0.42 \mathrm{MeV}$.

The CNO cycle is a chain of six reactions

$$
\begin{aligned}
& { }^{12} \mathrm{C}+\mathrm{p} \rightarrow{ }^{13} \mathrm{~N}+\gamma, \\
& { }^{13} \mathrm{~N} \rightarrow{ }^{13} \mathrm{C}+\mathrm{e}^{+}+v_{\mathrm{e}}, \\
& { }^{13} \mathrm{C}+\mathrm{p} \rightarrow{ }^{14} \mathrm{~N}+\gamma, \\
& { }^{14} \mathrm{~N}+\mathrm{p} \rightarrow{ }^{15} \mathrm{O}+\gamma, \\
& { }^{15} \mathrm{O} \rightarrow{ }^{15} \mathrm{~N}+\mathrm{e}^{+}+v_{\mathrm{e}} \\
& { }^{15} \mathrm{~N}+\mathrm{p} \rightarrow{ }^{12} \mathrm{C}+{ }^{4} \mathrm{He},
\end{aligned}
$$

where the second and sixth reactions are sources of neutrinos with energies

$$
E_{v}\left({ }^{13} \mathrm{~N}\right) \leqslant 1 \mathrm{MeV} \text { and } E_{v}\left({ }^{15} \mathrm{O}\right) \leqslant 1.5 \mathrm{MeV} .
$$

In addition, the proton-proton cycle contains an additional branch consisting of the reactions

$$
{ }^{7} \mathrm{Be}+\mathrm{e}^{-} \rightarrow{ }^{7} \mathrm{Li}+v
$$

and

$$
{ }^{8} \mathrm{~B} \rightarrow{ }^{8} \mathrm{Be}+\mathrm{e}^{+}+v,
$$

which are the sources of so-called beryllium neutrinos with energies $E_{\mathrm{v}}=0.86 \mathrm{MeV}(90 \%)$ and $E_{\mathrm{v}}=0.38 \mathrm{MeV}(10 \%)$ and high-energy boron neutrinos with an energy $E_{v} \leqslant 14$ $\mathrm{MeV}$, respectively (see Fig. 13a, which was taken from [134]). Two more components of the pp-cycle are also shown in Fig. 13a: the monoenergetic line representing the pep reaction $\mathrm{p}+\mathrm{e}+\mathrm{p} \rightarrow \mathrm{d}+\mathrm{v}$ at $E_{\mathrm{v}}=1.44 \mathrm{MeV}$, and the neutrinos generated in the hep reaction ${ }^{3} \mathrm{He}+\mathrm{p}$ with $E_{v} \leqslant 18.7 \mathrm{MeV}$.

Figure $13 \mathrm{a}$ shows that the fairly soft proton neutrinos $\left(E_{\mathrm{v}} \leqslant 0.42 \mathrm{MeV}\right)$ constitute the main part of the neutrino flux: there are approximately $10^{4}$ times more soft neutrinos than there are hard boron neutrinos $\left(E_{v} \leqslant 14 \mathrm{MeV}\right)$.

The first experiments of Davis et al. [131] in recording solar neutrinos at the Earth's surface were done via the reaction

$$
v+{ }^{37} \mathrm{Cl} \rightarrow{ }^{37} \mathrm{Ar}+\mathrm{e}^{-},
$$

with a $0.814 \mathrm{MeV}$ threshold $\dagger$.

Thus, the reaction (81) can be used to register only a small fraction of the solar neutrinos, which of course is inconvenient since it requires building huge detectors and using extremely long exposure times. Hence the more convenient radiochemical reaction for registering neutrinos is that in

$\dagger$ In 1946, Bruno Pontecorvo suggested using radiochemical methods in neutrino detection [17].
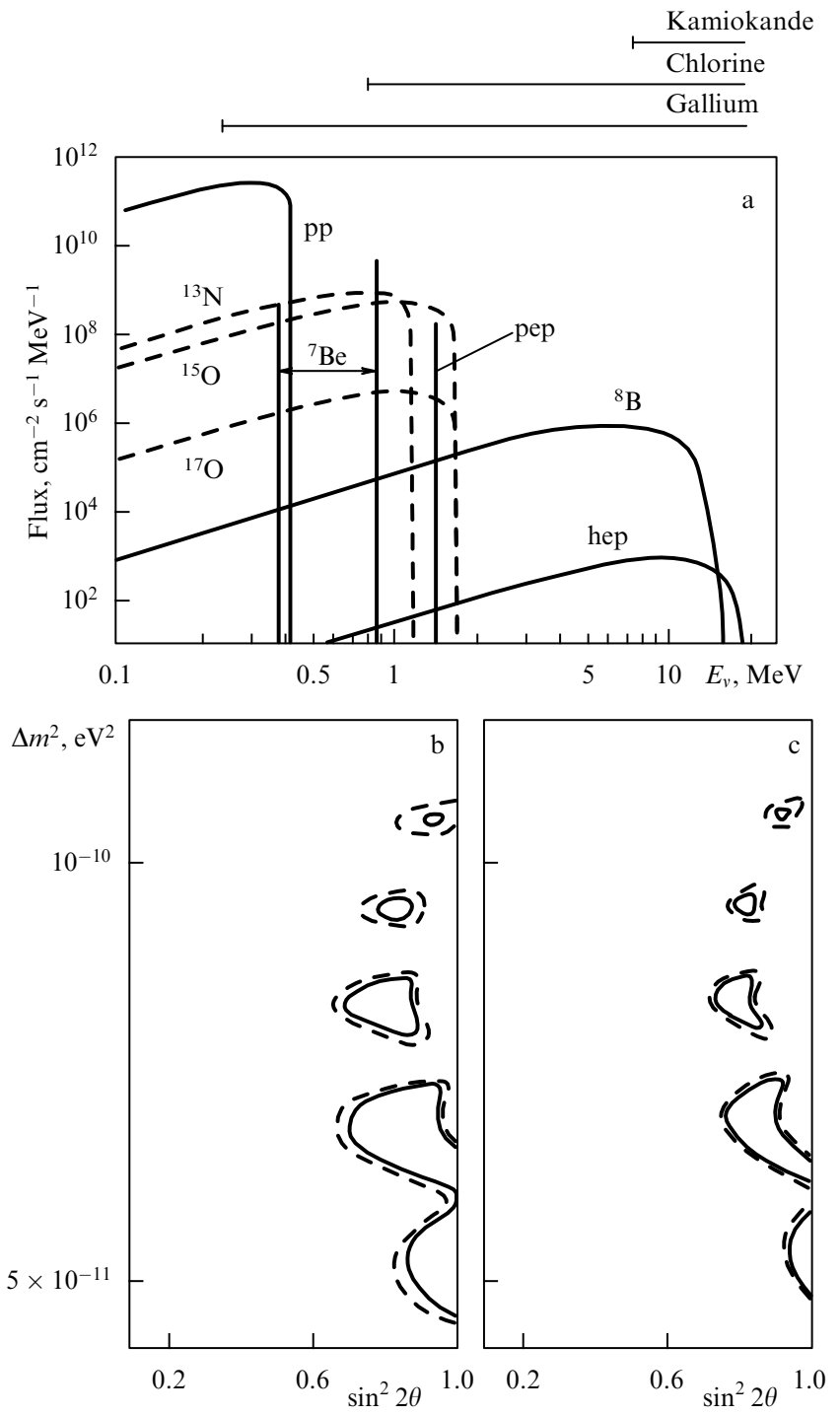

Figure 13. Solar neutrino parameters: (a) the neutrino flux density from the pp (solid curves) and CNO (dashed curves) cycles at the Earth's surface. For monoenergetic neutrinos the flux is measured in $\mathrm{cm}^{-2} \mathrm{~s}^{-1}$, and for neutrinos with a continuous energy spectrum the flux is measured in $\mathrm{cm}^{-2} \mathrm{~s}^{-1} \mathrm{MeV}^{-1}$. The sensitivity ranges for various detectors are shown at the top of the figure; (b) depicts the allowed parameters $\Delta m^{2}-\sin ^{2} 2 \theta$ for vacuum oscillations of solar neutrinos with allowing for the theoretical uncertainties in the analysis, and (c) depicts the ranges of the same parameters without taking into account the uncertainties.

which a neutrino interacts with the rare element $\mathrm{Ga}$ :

$$
v_{\mathrm{e}}+{ }^{71} \mathrm{Ga} \rightarrow{ }^{71} \mathrm{Ge}+\mathrm{e}^{-} \text {. }
$$

This reaction has an extremely low threshold, $E=0.23 \mathrm{MeV}$, which makes it possible to register the main part of the neutrino flux (thus the volume of the detector and the exposure time can be reduced). A further advantage of this reaction is that it is less dependent on the solar model, and in particular on the Sun's temperature, to which the fluxes of beryllium neutrinos $\left(\sim T_{\mathrm{C}}^{8}\right)$ and especially of boron neutrinos $\left(\sim T_{\mathrm{C}}^{18}\right)$ are extremely sensitive.

5.4.2 Solar neutrino deficit and the problem of beryllium neutrinos. The first experiments of Davis et al. [131] in registering solar neutrinos via reaction (81) were carried out 
in 1968 in a deep $(1.5 \mathrm{~km})$ South Dakota gold mine. The detector consisted of $610 \mathrm{t}$ of tetrachloroethylene $\mathrm{C}_{2} \mathrm{Cl}_{4}$ that occupied a volume of 380 cubic meters. By complicated technological procedures an extremely small amount of radioactive ${ }^{37} \mathrm{Ar}(0.3 \pm 0.2$ atom per day) was periodically extracted from the huge target volume, and this amount was compared to the results of calculations based on the generally accepted thermonuclear model of the Sun.

It was found that the observed effect was approximately three times weaker than the expected effect. There was not a single solar model that could eliminate this imbalance (including the hypothesis that solar energy is not produced in thermonuclear reactions!). It was then that Gribov and Pontecorvo [130] proposed the idea of oscillations of solar neutrinos, according to which a fraction of the $v_{\mathrm{e}}$ transforms into $v_{\mu}$ on their way from the Sun to Earth (nothing was known about the tau neutrino at the time), and thus they cannot be registered in the reaction (81).

The discovered solar neutrino deficit repeatedly manifested itself in all Davis and his collaborators' subsequent experiments. Up-to-date data obtained by the chlorine method in the Homestake experiment [135] showed that only $27.5 \%$ of the calculated number of neutrinos were registered by the detector.

The solar neutrino deficit was also registered in other experiments, which used entirely different methods. Two of these experiments, GALLEX [136, 137] and SAGE [138, 139], used reaction (82), which made it possible to register the main fraction of the flux of neutrinos of relatively moderate energies. As in the first case, the researchers used the radiochemical approach to extracting the reaction products.

In the GALLEX Collaboration (the underground Gran Sasso d'Italia laboratory), the detector consisted of $30 \mathrm{t}$ of gallium contained in $101 \mathrm{t}$ of water solution of gallium chloride. The volatile gallium tetrachloride that formed as a result of irradiation of the detector by solar neutrinos was extracted from the solution and processed into germanium hydride. The product was then used together with xenon to fill the proportional counters (Auger electrons and X-rays from electron capture, with energies of 10.4 and $1.2 \mathrm{keV}$, were detected).

The SAGE experiment (a Russian-American Collaboration) took place at the Baksan Neutrino Laboratory at a depth of 4800-m water equivalent in Caucasia. The researchers used $55 \mathrm{t}$ of gallium contained in eight chemical reactors. Prior to the irradiation, approximately $700 \mathrm{mg}$ of natural Ge was added to the gallium as a carrier, in the form of a $\mathrm{Ge}-\mathrm{Ga}$ impurity. After irradiation was completed, the product nuclei of ${ }^{71} \mathrm{Ge}$ were extracted by chemical methods together with the carrier. The rest is similar to the procedure followed in the GALLEX experiment.

Both experiments confirmed the existence of a solar neutrino deficit, with a $57 \%$ ratio of the measured number of neutrinos to the calculated number in the GALLEX experiment and a $51 \%$ ratio in the SAGE experiment.

Another experiment in detecting solar neutrinos was conducted somewhat earlier [140]. It involved the large Cherenkov water detector Kamiokande, mentioned in Section 3.2.5. What was measured was the angular distribution of elastically scattered electrons in the process $v_{\mathrm{e}}+\mathrm{e}^{-} \rightarrow v_{\mathrm{e}}+\mathrm{e}^{-}$. The events that were selected corresponded to the emission of electrons at a small angle to the Sun-Earth direction. The idea of the method was based on the fact that the process of $v_{\mathrm{e}} \mathrm{e}$-scattering involves charged and neutral currents, while the $v_{\mu} \mathrm{e}$ - and $v_{\tau} \mathrm{e}$-scattering processes involve only neutral currents, and because of this $\sigma\left(v_{\mathrm{e}} \mathrm{e}\right)>\sigma\left(v_{\mu} \mathrm{e}\right)$ and $\sigma\left(v_{\mathrm{e}} \mathrm{e}\right)>\sigma\left(v_{\tau} \mathrm{e}\right)$. The detection threshold for the scattered electrons was $7.5 \mathrm{MeV}$ (see the upper part of Fig. 13a). The number of neutrinos detected in the measurements amounted to $44 \%$ of the calculated number $\dagger$.

Thus, in all four experiments the detectors registered less than half the number of solar neutrinos in comparison to the calculated value that follows from the Standard Solar Model. However, serious difficulties arise when one tries to study all these experiments together.

On the one hand, the Standard Solar Model provides a fairly good picture of the spectral composition of solar neutrinos (Fig. 13a) and allows an estimate of the effect of the individual components in the neutrino spectrum in different detectors. For instance, the effect of the ${ }^{7} \mathrm{Be}-$ neutrino in the gallium detector must amount to $34 \mathrm{SNU}$ (solar-neutrino unit), while in a chlorine detector it amounts to $1.1 \mathrm{SNU}$, where $1 \mathrm{SNU}=10^{-36}$ neutrino captures per atom per second.

On the other hand, by combining the results of measurements made on different detectors (chlorine, gallium, and Kamiokande) one can isolate the experimental magnitudes of the same effects. For instance, the combination of the gallium method and Kamiokande yields $-4_{-7}^{+11} \mathrm{SNU}$ for the ${ }^{7} \mathrm{Be}-$ neutrino, while the combination of the chlorine method and Kamiokande yields $-0.66 \pm 0.58 \mathrm{SNU}$ for the $\left({ }^{7} \mathrm{Be}+\right.$ pep + $\mathrm{CNO}$ )-neutrino. Thus, the combination of all four experiments shows that there should be no ${ }^{7} \mathrm{Be}$-neutrinos in the solar-neutrino spectrum.

This discrepancy became known as the solar-neutrino problem, which allows four possible alternative interpretations.

1. The flux of ${ }^{7} \mathrm{Be}$-neutrinos detected at the Earth's surface is indeed considerably weaker (at least by a factor of two) than that predicted by the Standard Solar Model. A possible reason for such a decrease in intensity is the vacuum oscillations of the ${ }^{7}$ Be-neutrinos accompanied by a transformation of these neutrinos into $v_{\mu}$ and/or $v_{\tau}$. If this assumption is correct, the allowed regions in the $\Delta m^{2}-\sin ^{2} 2 \theta$ plane are those depicted in Figs. 13b and c [141]. Notice that it is practically impossible to verify this hypothesis by seasonal measurements of neutrino fluxes because the eccentricity of the Earth's orbit is so small $(\sim 0.017)$.

2. The Standard Solar Model is correct, but the MikheevSmirnov-Wolfenstein effect is present (see Sections 5.4.3 and 5.4.4).

3. The Standard Solar Model is correct, but the ${ }^{7} \mathrm{Be}-$ neutrinos reorient into sterile neutrinos because of the interaction between the neutrino magnetic moment and the solar magnetic field (see Sections 5.4.5 and 5.4.6).

4. The Standard Solar Model is correct and nothing happens to the neutrinos (there are no oscillations and no magnetic reorientation), but three of the four experiments described above are incorrect.

In any case, it is highly important that new measurements be made, especially a more direct experiment to estimate the ${ }^{7}$ Be-neutrino flux, which is expected to be done using the Borexino facility (see Section 6.4).

$\dagger$ By April 1997, the Superkamiokande device (see Section 6.2) measured a solar neutrino flux over the course of 201.6 days of on-time, which in comparison to the neutrino flux calculated by the Standard Solar Model amounted to $0.400_{-0.012}^{+0.013}$ (stat $)_{-0.014}^{+0.020}$ (calc) with a $6.5 \mathrm{MeV}$ threshold. Here no night-day variations in flux (mentioned earlier) were detected. 
5.4.3 Wolfenstein equations. In Section 5.4.2 the solar neutrino deficit was explained by vacuum oscillations, which ignore the fact that the solar neutrinos partially travel through extremely dense solar matter. At the same, if we continue the analogy with oscillations of $\mathrm{K}^{0}$-mesons, we must note that when the mesons pass through dense matter, the phenomenon of coherent regeneration of $\left(\mathrm{K}^{0}-\widetilde{\mathrm{K}}^{0}\right)$ - and $\left(\mathrm{K}_{L}^{0}-\mathrm{K}_{S}^{0}\right)$-mesons emerges with a maximum amplitude of mutual transformation.

In 1977 - 1978, Wolfenstein $[142,143]$ used this analogy to derive a system of temporal equations describing the evolution of the wave functions $\psi_{f}=\left(\psi_{v_{\mathrm{e}}}, \psi_{v_{\mu}}\right)$ :

$$
\mathrm{i} \frac{\mathrm{d} \psi_{f}}{\mathrm{~d} t}=\left(p \hat{I}+\frac{\widehat{M}^{2}}{2 p}+\widehat{W}\right) \psi_{f} .
$$

This system of equations is a generalization of the Schrödinger equation for a single particle of mass $m$, kinetic energy $E$ (assuming that $m \ll p$ and $\hbar=c=1$ ), and potential energy $W$ :

$$
\mathrm{i} \frac{\mathrm{d} \psi}{\mathrm{d} t}=(E+W) \psi \approx\left(p+\frac{m^{2}}{2 p}+W\right) \psi
$$

in which $m^{2}$ must be replaced by $\widehat{M}^{2}$ (the square of the mass matrix of the neutrino in a vacuum), and $W$ by the matrix $\widehat{W}$ (which takes into account the neutrino-matter interaction), and $p$ is written as $p \hat{I}$, with $\hat{I}$ the identity matrix.

This system of equations was examined in an approximation of the medium with a constant density, which made it possible to obtain some of the new features of neutrino oscillations in the Earth, the Sun, and collapsing stars. It was found that because of the difference in the interaction of the $v_{\mathrm{e}}$ and $v_{\mu}$ with different components of the medium, the medium had different refractive indices in relation to the waves describing the propagation of these neutrinos. Ultimately, this leads to a situation in which the process of mixing of $v_{\mathrm{e}}$ and $v_{\mu}$ incorporates new mass states of $v_{1}^{\prime}$ and $v_{2}^{\prime}$, which differ from $v_{1}$ and $v_{2}$. Even the oscillation length and the nature of oscillations change: the oscillations in the medium may become stronger or weaker.

5.4.4 The Mikheev-Smirnov-Wolfenstein effect. In 19841985, Mikheev and Smirnov (see the review [144]) studied neutrino oscillations in a media with a varying density, which is characteristic, for example, of the Sun. As a result of their research they found [145-147] that the dependence of the parameter $\sin ^{2} 2 \theta^{\prime}$, where $\theta^{\prime}$ is the mixing angle in the medium, on the medium's density $\rho$ (or the neutrino energy) is of a resonant nature. At resonant values $\rho_{\text {res }}$ (or $E_{\text {res }}$ ) the mixing is at its maximum and new effects become evident [145-148]. Two of them are discussed here.

In the process of neutrino motion, the depth of neutrino oscillations and the average magnitude of such oscillations vary. Within a broad energy interval there can be practically complete transitions of one type of neutrino into another even at small mixing angles (compare with the case of a vacuum, where complete transitions occur only at the maximum mixing angle $\theta=45^{\circ}$ ).

The second important effect consists in the following. In contrast to a vacuum or a medium with constant density, where intense transformations $v_{\mathrm{e}} \leftrightarrow v_{\mu}$ occur only at discrete energies, in a medium with variable density such transformations may occur over a continuous energy range. Here, if the initial density $\rho$ is much higher than $\rho_{\text {res }}$, the transformation of one type of neutrino into another may take place practically without oscillations as the density varies [147].

These effects become possible if, in addition to the resonance condition being met, the medium's density varies very slowly (the variation resembling the adiabatic mode). Here the layer of dense matter must be sufficiently thick [144], i.e. the new theory can be used in neutrino cosmology, astrophysics, and geophysics.

Specifically, the possible areas of research may be collapsing stars, the Sun, and the Earth, where because of the resonance effect the parameters $\Delta m^{2}$ and $\sin ^{2} 2 \theta$ may vary by several orders of magnitude. In particular, resonant oscillations of solar neutrinos may initiate the formation of regions with $\Delta m^{2} \leqslant 10^{-4} \mathrm{eV}$, where the loss of $v_{\mathrm{e}}$ may increase by a factor of two to four, thus explaining Davis's measurements. If this is true, the generally accepted shape of the solarneutrino energy spectrum must change. This can be verified by carrying out a complete neutrino spectroscopy of the Sun (see Section 6.4). This problem, important by itself, has a curious consequence in particle physics. The thing is that the region of values of $\Delta m^{2}$ mentioned above, where, possibly, the number of $v_{\mathrm{e}}$ rapidly decreases, is of order $\Delta m^{2} \leqslant 10^{-4} \mathrm{eV}$. This means that if there is a hierarchy of neutrino masses, then $m_{v_{\mathrm{e}}} \ll 10^{-2} \mathrm{eV}$ !

Thus, if the Mikheev-Smirnov-Wolfenstein resonance effect does exist, it may lead to a very accurate estimate of an upper bound on the neutrino mass.

5.4.5 Hypothesis of the existence of a neutrino magnetic moment. In 1986, Voloshin et al. [149] and Veselov et al. [150] hypothesized that the reason for the solar neutrino deficit was the precession of the magnetic moments of neutrinos in the variable fields of the Sun, a precession that may lead to the transformation of an ordinary left-handed neutrino $v_{\mathrm{e}}^{L}$ into a sterile right-handed neutrino $v_{\mathrm{e}}^{R}$, which has not been registered in Davis's experiments. Their hypothesis seemed to be corroborated by the discovered anticorrelation between variations in the solar neutrino flux at the Earth's surface and the number of solar spots, related to changes in solar activity and variations of the magnetic field at the surface of the Sun.

In 1994, Oakley et al. [151] analyzed a more direct anticorrelation between the solar-neutrino count rate in the Homestake experiment (see Section 5.4.2) and the results of measurements of the magnetic field at the Sun's surface. Thus, the hypothesis expressed in $[149,150]$ received strong support.

For the effect discussed to appear, the neutrino magnetic moment must be of order

$$
\mu_{v} \simeq 10^{-11} \mu_{\mathrm{B}}
$$

where $\mu_{\mathrm{B}}=e \hbar / 2 m_{\mathrm{e}} c$ is the electron Bohr magneton. However, the neutrino magnetic moment predicted by the Standard Model of the electroweak interaction is many orders of magnitude smaller than the above value [149, 152]. Hence the discovery of a neutrino magnetic moment of order $10^{-11} \mu_{\mathrm{B}}$ would not strongly support the hypothesis of the origin of the solar neutrino deficit but it would also contribute considerably to the physics of electroweak interactions (generalization of the Standard Model).

It is possible, at least in principle, to discover the neutrino magnetic moment by studying the $(v-\mathrm{e})$-scatter- 
ing of reactor antineutrinos. The goal is to establish, against the background of normal weak scattering, a contribution to scattering due to the magnetic moment. Such experiments require a knowledge of the spectrum of the antineutrinos emitted by the fission fragments and radioactive atoms produced in the reactor as a result of radiative neutron capture. The spectra of antineutrinos with $E_{\tilde{v}}>2 \mathrm{MeV}$ (roughly 25\%) were obtained by Schreckenbach et al. [153] and Hahn et al. [154] by converting the beta spectra measured and by Vogel et al. [155] via calculations. The spectra with $E_{\tilde{v}}<2 \mathrm{MeV}$ (roughly $75 \%$ ) were obtained for the fission fragments by Aleksankin et al. [156], Rubtsov et al. [157], and Vogel and Engel [152], and for the radioactive nuclei that form as a result of neutron capture, by Mikaelyan and collaborators [158, 159]. In the last preprints the authors also give the complete antineutrino spectra for reactors of different types.

Measurements of $(\tilde{v}-\mathrm{e})$-scattering were made with reactor antineutrinos from the Savannah-River [160] and Krasnoyarsk [161] reactors mentioned earlier and from the Rovno (Ukraine) nuclear power plant [162]. They yielded the following upper bound on the magnetic moment of the neutrino [163]:

$$
\mu_{v}<1.8 \times 10^{-10} \mu_{\mathrm{B}}
$$

which is approximately 20 times larger than the value needed to explain the solar neutrino deficit.

The problem of further reducing an upper bound on $\mu_{v}$ was discussed in Refs [158, 159], where for a free electron at rest the differential scattering cross section of a neutrino with a magnetic moment $\mu_{v}[164]$ is compared with the differential cross section of weak scattering (e.g., see Ref. [149]) at different neutrino energies $E_{v}$ and different kinetic energies of recoil electrons, $T_{\mathrm{e}}$. The results of the calculations suggest that the sensitivity of the $(v-\mathrm{e})$-scattering experiments to the neutrino magnetic moment increases with a decrease in the energy $T_{v}$ of the recoil electrons. To detect $\mu \simeq 10^{-11} \mu_{\mathrm{B}}$ against the weak-scattering 'background', we must be able to register recoil electrons with an energy $\leqslant 10 \mathrm{keV}$.

Just how complex such a problem is becomes evident when we consider the fact that devices capable of registering recoil electrons with energies of several hundreds of $\mathrm{keV}$ are still being designed. When such devices become operational, they could reduce the value specified in (86) severalfold: to $4 \times 10^{-11} \mu_{\mathrm{B}}$ at $T_{\mathrm{e}} \simeq 200 \mathrm{keV}$, and to $3 \times 10^{-11} \mu_{\mathrm{B}}$ at $T_{\mathrm{e}} \simeq 100 \mathrm{keV}$.

5.4.6 Hypothesis of spin-flavour precession of neutrinos. In 1987, Akhmedov and Khlopov [165] proposed a mechanism of resonant amplification of the transitions between neutrino states of different flavours, even if the mixing of these states in a vacuum is weak, when a neutrino that has a magnetic moment (including a transitional moment) travels through a longitudinal magnetic field. They called this mechanism spin-flavour precession. This idea was thereafter developed by Akhmedov and Bychuk [166], who studied the effect of this mechanism on the passage of neutrinos inside the Sun. Akhmedov et al. [167] calculated the possible effects of spin-flavour neutrino precession for nine different profiles of the solar magnetic field. They also predicted the rates of neutrino event counts for experiments planned for the near future, such as SNO, Superkamiokande, and Borexino (see Section 6).

\subsection{Search for atmospheric neutrino oscillations.}

\section{The atmospheric neutrino anomaly}

Atmospheric neutrinos are formed in the $(\pi \rightarrow \mu \rightarrow \mathrm{e})$-decay of $\pi^{ \pm}$-mesons and the $\left(\mathrm{K} \rightarrow \mu v_{\mu}\right)$ - and $\left(\mathrm{K} \rightarrow \mu \pi^{0} v_{\mu}\right)$-decays of $\mathrm{K}^{ \pm}$-mesons created in primary cosmic processes.

Atmospheric neutrinos have energies in the $10-100 \mathrm{GeV}$ range, and they may travel extremely large distances (from the other side of the Earth) before reaching the detector or originate in the nearest layers of the Earth's atmosphere.

The idea of searching for atmospheric neutrino oscillations consists in comparing the measured ratio of the numbers of muon and electron neutrinos and antineutrinos to the ratio of the same numbers calculated by the Monte Carlo method:

$$
\left[\frac{N\left(v_{\mu}+\tilde{v}_{\mu}\right)}{N\left(v_{\mathrm{e}}+\tilde{v}_{\mathrm{e}}\right)}\right]_{\exp } /\left[\frac{N\left(v_{\mu}+\tilde{v}_{\mu}\right)}{N\left(v_{\mathrm{e}}+\tilde{v}_{\mathrm{e}}\right)}\right]_{\text {calc }} .
$$

The main decay schemes imply that there must be twice as many muon neutrinos and antineutrinos as there are electron neutrinos and antineutrinos. It occurs that this ratio is modelindependent (i.e. independent of the shower model) and is valid over a broad interval of neutrino energies, $0.1 \leqslant E_{v} \leqslant 1 \mathrm{GeV}$. Hence, comparison of the measured ratio of the numbers of $v_{\mu}$ and $v_{\mathrm{e}}$ with the normalized calculated ratio (which takes into account all the intricacies in detecting both types of neutrinos) serves as a good instrument in the search for $v_{\mu}$ and $\tilde{v}_{\mu}$ oscillations.

The ratio (87) was measured in 1992 in the Kamiokande experiment [168]:

$$
R=\left(\frac{N_{\mu}}{N_{\mathrm{e}}}\right)_{\exp } /\left(\frac{N_{\mu}}{N_{\mathrm{e}}}\right)_{\text {calc }}=0.6_{-0.06}^{+0.07} .
$$

This result reflects what is known as the atmospheric neutrino anomaly and can be interpreted as loss of a fraction of the muon neutrinos because of $\left(v_{\mu} \leftrightarrow v_{\mathrm{e}}\right)$ - and $\left(v_{\mu} \leftrightarrow v_{\tau}\right)$ oscillationst.

In view of the fact that atmospheric neutrinos can enter a detector either from above, travelling a fairly short distance $R_{1} \simeq 10^{4} \mathrm{~m}$, or from below, by first travelling through the entire globe a distance $R_{2} \simeq 1.2 \times 10^{7} \mathrm{~m}$ ), the parameters of the possible oscillations must depend on the zenith angle of the interacting neutrinos. In 1994, Fukuda et al. [169] analyzed this situation and found that the ratio (87) diminishes by a factor of approximately four (from $\sim 1.2$ to $\sim 0.3$ ) as the zenith angle grows from 0 to $180^{\circ}$. Their analysis made it possible to obtain the parameters of possible $\left(v_{\mu} \leftrightarrow v_{e}\right)$ - and $\left(v_{\mu} \leftrightarrow v_{\tau}\right)$-oscillations, which are depicted in Fig. 14 for neutrinos with energies lower and higher than 1 $\mathrm{GeV}$ separately (dashed and heavy solid curves, respectively). Clearly, the lower limits $\Delta m^{2} \simeq 10^{-3} \mathrm{eV}^{2}$ correspond to registering 'antipode' neutrinos $\left(R \simeq 1.2 \times 10^{7} \mathrm{~m}\right)$, while the upper limits $\Delta m^{2} \simeq 10^{-1} \mathrm{eV}^{2}$ correspond to registering 'zenith' neutrinos $\left(R \simeq 10^{4} \mathrm{~m}\right)$.

Muon neutrinos from the other side of the globe were also registered at the Baksan Neutrino Laboratory by Boliev et al. [170]. The researchers obtained $0.98 \pm 0.20$ for the ratio of the flux of registered $v_{\mu}$ to the expected flux,

$\dagger$ By April 1997, the Superkamiokande device (see Section 6.2) had produced a value for the ratio (87) equal to $0.52 \pm 0.09 \pm 0.06$ (in the multi-GeV range), with the result being uniform over the entire volume of the detector, so that it cannot be explained by a possible neutron effect. 

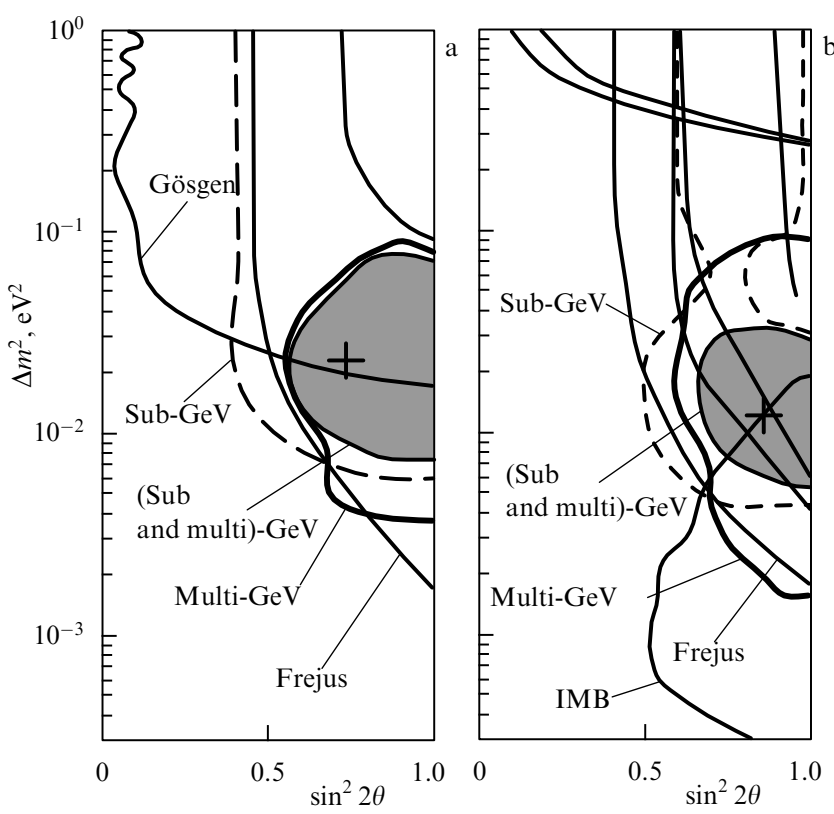

Figure 14. Allowed regions of parameters for neutrino oscillations obtained in the Kamiokande experiment for the $v_{\mu} \leftrightarrow v_{\mathrm{e}}$ (a) and $v_{\mu} \leftrightarrow v_{\tau}$ (b) hypotheses and plotted in the $\Delta m^{2}-\sin ^{2} 2 \theta$ plane. The crosses indicate the most probable values of the parameters. The solid light curves limit the regions that have been excluded by other experiments.

which yields

$$
\begin{aligned}
& \Delta m^{2}<6 \times 10^{-3} \mathrm{eV}^{2} \text { at } \sin ^{2} 2 \theta=1, \\
& \sin ^{2} 2 \theta<0.65 \text { for large } \Delta m^{2} .
\end{aligned}
$$

In other experiments the ratio (87) was also found to differ by a factor of approximately two. Values close to those obtained in the Kamiokande experiment (0.6) were obtained via the Cherenkov water detector IMB [171] and the electron detector (gaseous counters) SUDAN-2 [172], and values close to unity were obtained from the Frejus electron detector with iron plates [173] and the similar NUSEX detector [174] (see Table 1).

Thus, although Fig. 14 shows that almost the entire range of parameters obtained in the Kamiokande experiments is cut off by the data of other experiments, the problem of the atmospheric neutrino anomaly has yet to

Table 1

\begin{tabular}{lll}
\hline Experiment & Result & Source \\
\hline $\begin{array}{l}\text { Kamiokande } \\
\text { (sub-GeV) }\end{array}$ & $0.60_{-0.06}^{+0.07} \pm 0.05$ & {$[168]$} \\
$\begin{array}{l}\text { Kamiokande } \\
\text { (multi-GeV) }\end{array}$ & $0.67_{-0.07}^{+0.08} \pm 0.07$ & {$[169]$} \\
Baksan & $0.98 \pm 0.20$ & \\
IMB & $0.54 \pm 0.05 \pm 0.12$ & {$[171]$} \\
SUDAN-2 & $0.69 \pm 0.19 \pm 0.09$ & {$[172]$} \\
Frejus & $1.06 \pm 0.18 \pm 0.15$ & {$[173]$} \\
NUSEX & $0.99_{-0.25}^{+0.35}$ & {$[174]$} \\
\hline
\end{tabular}

be solved, in view of which new measurements are essential (see Section 6).

\subsection{Search for accelerator neutrino oscillations in experiments with long and short baselines}

In view of the different features of the reactor experiments involved in the search for neutrino oscillations, it is more convenient to study each type of reactor experiment separately.

5.6.1 Experiments involving high-energy accelerators with a long baseline. The search for neutrino oscillations in experiments involving accelerators with long baselines was conducted using the high-energy accelerators at CERN and the National Accelerator Laboratory near Batavia, Illinois. A specific feature of these experiments was the registering of neutrino events of the type $v_{\mu}+N \rightarrow \mu^{-}+X$ by two detectors simultaneously.

One experiment was conducted at CERN by the CDUS group [175], with the detectors placed 130 and $885 \mathrm{~m}$ away from the source of muon neutrinos whose energies were about $1 \mathrm{GeV}$. The results of the experiment revealed that oscillations cannot be observed in the intervals

$$
\begin{aligned}
& 0.26 \leqslant \Delta m^{2} \leqslant 90 \mathrm{eV}^{2} \text { at } \sin ^{2} 2 \theta=1, \\
& \sin ^{2} 2 \theta \geqslant 0.053 \text { at } \Delta m^{2} \simeq 2.5 \mathrm{eV}^{2}
\end{aligned}
$$

Another experiment was also conducted at CERN by the CHARM group [176], with the detectors placed 123 and $903 \mathrm{~m}$ away from the source of muon neutrinos. The experiment yielded the following values of $\Delta m^{2}$ and $\sin ^{2} 2 \theta$ at which there can be no oscillations:

$$
\begin{aligned}
& 0.29 \leqslant \Delta m^{2} \leqslant 22 \mathrm{eV}^{2} \text { at } \sin ^{2} 2 \theta=1, \\
& \sin ^{2} 2 \theta \geqslant 0.20 \text { at } \Delta m^{2}=2 \mathrm{eV}^{2} .
\end{aligned}
$$

The CCFR group [177] at Batavia found the widest range of values of $\Delta m^{2}$ and the lowest value of $\sin ^{2} 2 \theta$ in the region checked for absence of oscillations. In their experiment the detectors were placed 715 and $1116 \mathrm{~m}$ away from the source of $40-230-\mathrm{GeV}$ neutrinos. The results were

$$
\begin{aligned}
& 15 \leqslant \Delta m^{2} \leqslant 1600 \mathrm{eV}^{2} \text { at } \sin ^{2} 2 \theta=1, \\
& \sin ^{2} 2 \theta \geqslant 0.002 \text { at } \Delta m^{2} \simeq 100 \mathrm{eV}^{2} .
\end{aligned}
$$

Another record-breaking result for the size of the studied forbidden range of values of $\Delta m^{2}\left(0,5-10^{3} \mathrm{eV}^{2}\right)$ and values of $\sin ^{2} 2 \theta\left(3 \times 10^{-3}\right)$ was obtained at the Brookhaven National Laboratory (BNL), Upton, New York [178]. A remarkable feature of this experiment, in which $1.5-\mathrm{GeV}$ neutrinos were registered at a distance of $100 \mathrm{~m}$, was the detection of the quasi-elastic processes

$$
v_{\mu}+\mathrm{n} \rightarrow \mu^{-}+\mathrm{p}, \quad v_{\mathrm{e}}+\mathrm{n} \rightarrow \mathrm{e}^{-}+\mathrm{p},
$$

which made it possible to study the $v_{\mu}$ to $v_{\mathrm{e}}$ flux ratio, i.e. to investigate specific oscillations, $\left(v_{\mu} \leftrightarrow v_{\mathrm{e}}\right)$. The BNL experiment revealed the following limits for the regions in which oscillations are possible:

$$
\begin{aligned}
& \Delta m^{2} \leqslant 0.5 \mathrm{eV}^{2} \text { at } \sin ^{2} 2 \theta=1, \\
& \sin ^{2} 2 \theta<3 \times 10^{-3} \text { at } \Delta m^{2} \simeq 10 \mathrm{eV}^{2} .
\end{aligned}
$$

A later estimate obtained in this experiment for $\sin ^{2} 2 \theta=1$ was $\Delta m^{2} \leqslant 0.09 \mathrm{eV}^{2}$. 
5.6.2 Experiments involving high-energy accelerators with a short baseline. If we place a beam absorber (a copper tank filled with water) in the path of high-energy pions and muons, whose length is sufficient for stopping the particles completely, such a device (called a copper beam stop) will serve as a source of $v_{\mu}$ (if the stopped $\pi^{+}$-mesons decay according to the scheme $\pi^{+} \rightarrow \mu^{+}+v_{\mu}$ ) as well as $\tilde{v}_{\mu}$ and $v_{\mathrm{e}}$ (if the stopped $\mu^{+}$muons decay according to the scheme $\mu^{+} \rightarrow \mathrm{e}^{+}+v_{\mathrm{e}}+\tilde{v}_{\mu}$ ).

Then, if we position a detector near the neutrino source, we are ready to study oscillations of $v_{\mu}$ and $\tilde{v}_{\mu}$ involving a short baseline. Experiments of this type were conducted at two facilities - the Los Alamos Meson Physics Facility (LAMPF): the LSND experiment [179], and at the Rutherford laboratory [180], where the $\pi^{+}$-mesons were generated by the ISIS proton beam: the KARMEN experiment [181, 182]. In the first case the detector was placed at a distance of $30 \mathrm{~m}$ from the source of neutrinos, and in the second, at a distance of $17.5 \mathrm{~m}$.

The Liquid Scintillator Neutrino Detector (LSND) is a big tank, $8.7 \mathrm{~m}$ long and $5.7 \mathrm{~m}$ in diameter, positioned at an angle of $12^{\circ}$ in relation to the proton beam. The inner surface of the tank hosts 1220 eight-inch photomultipliers, and the tank is filled with a liquid scintillator of such a composition and concentration that both Cherenkov radiation and scintillations can be detected. This made it possible to register the spatial-temporal sequence of signals for a positron and a gamma quantum generated in the following reactions:

$$
\tilde{v}_{\mathrm{e}}+\mathrm{p} \rightarrow \mathrm{n}+\mathrm{e}^{+}, \quad \mathrm{n}+\mathrm{p} \rightarrow \mathrm{d}+\gamma .
$$

The goal of the experimenters was to detect the $\tilde{v}_{\mathrm{e}}$ generated in a $\left(\tilde{v}_{\mu} \rightarrow \tilde{v}_{\mathrm{e}}\right.$ )-type oscillation process (an occurrence experiment). Athunassopoulos et al. [179] allowed for background events ( $2.1 \pm 0.3$ events) and discovered an excess amounting to 6.9 events. If this is interpreted as proof of the existence of oscillations of the $\tilde{v}_{\mu} \leftrightarrow \tilde{v}_{\mathrm{e}}$ type, the range of possible values of $\Delta m^{2}$ and $\sin ^{2} 2 \theta$ can be represented by the shaded band in Fig. 15. However, it should be said in all fairness that an alternative analysis done by Hill [183] showed that there was no excess in events above the background.

The design of the detector used in the KarlsruheRutherford Medium Energy Neutrino (KARMEN) experiment was similar to that of the LSND detector, but in contrast it was divided into 512 independent long $(350 \times 18 \times 18 \mathrm{~cm})$ modules insulated by layers of paper coated with $\mathrm{Gd}_{2} \mathrm{O}_{3}$. Each module was 'covered' by two photomultipliers at the ends of the module. The additional absorption of neutrons in Gd by the scheme

$$
\mathrm{Gd}+\mathrm{n} \rightarrow \mathrm{Gd}^{*}, \quad \mathrm{Gd}^{*} \rightarrow \mathrm{Gd}+\gamma
$$

presents a convenient way of isolating spatial-temporal $\mathrm{e}^{+} \gamma$ coincidences. The device also made it possible to study $\left(v_{\mu} \leftrightarrow v_{\mathrm{e}}\right)$-type oscillations by identifying the spatial-temporal coincidences of $\mathrm{e}^{+}$and $\gamma$ generated in the following reactions:

$$
v_{\mathrm{e}}+{ }^{12} \mathrm{C} \rightarrow{ }^{12} \mathrm{~N}^{*}+\mathrm{e}^{-}, \quad{ }^{12} \mathrm{~N}^{*} \rightarrow{ }^{12} \mathrm{~N}+\gamma .
$$

No events above the background were discovered. The use of the data of this experiment and of other experiments [184, 185] substantially narrows the shaded area in Fig. 15, leaving only a narrow strip below the dashed curve.

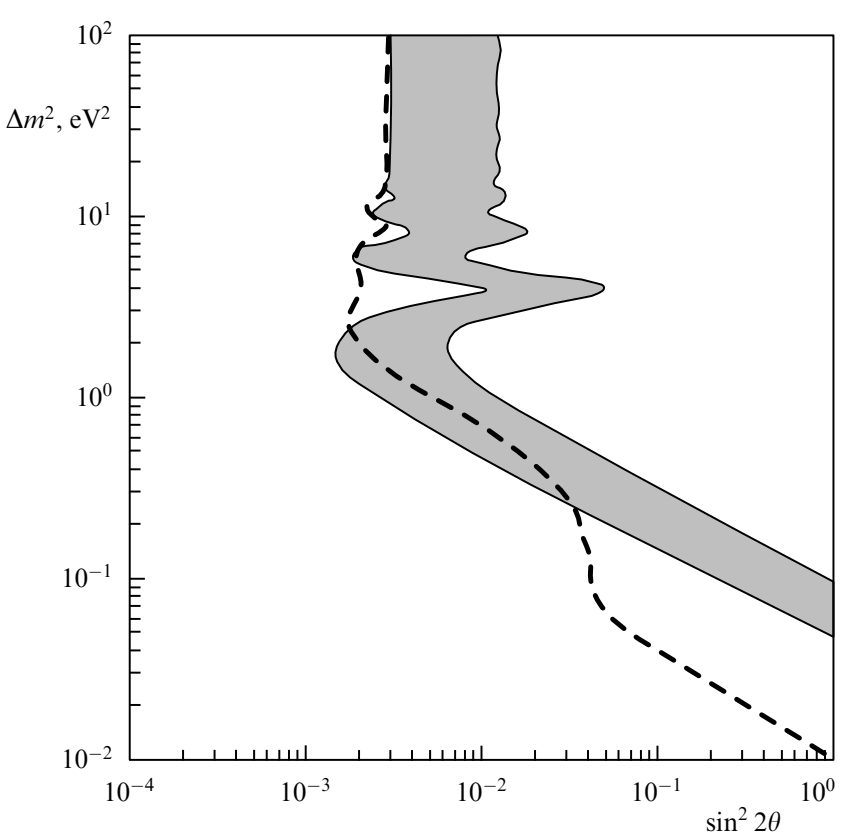

Figure 15. Results of the LSND experiment.

\subsection{Search for reactor antineutrino oscillations: historical survey and current experiments}

The story of the search for reactor antineutrino oscillations is just as dramatic as the unfinished story of the search for solar neutrino oscillations, where new hopes have emerged. There was a time, however, when the situation with reactor antineutrinos was even more dramatic than with solar neutrinos: twice reaction antineutrino oscillations were 'discovered' and twice the discoveries proved to be false. Below is a brief story of the search for reactor antineutrino oscillations (for details see the book by Borovoi and Khakimov [13]) and of the ongoing reactor experiments in this field.

5.7.1 First 'discovery' of reactor antineutrino oscillations. In 1980, Reines and collaborators published a paper [186] that analyzed an earlier work by Pasierb et al. [187] devoted to the interaction of electron antineutrinos and deuterons $\uparrow$ :

$$
\begin{aligned}
& \tilde{v}_{\mathrm{e}}+\mathrm{d} \rightarrow \mathrm{n}+\mathrm{n}+\mathrm{e}^{+}, \\
& \tilde{v}_{\mathrm{e}}+\mathrm{d} \rightarrow \mathrm{n}+\mathrm{p}+\tilde{v}_{\mathrm{e}} .
\end{aligned}
$$

The idea of using these reactions in the search for oscillations boils down to comparing the experimental ratio $R_{\exp }$ of the number of events detected in reactions (95) and (96) with the calculated ratio $R_{\text {calc }}$ for the same reaction but computed under the assumption that no oscillations are present. If a fraction of the $v_{\mathrm{e}}$ is transformed as a result of oscillations into $\tilde{v}_{\mu}$ or $\tilde{v}_{\tau}$, the number of events in reaction (95), which can proceed only under the action of $\tilde{v}_{\mathrm{e}}$, must decrease and in reaction (96), which is insensitive to the type of antineutrino, remains the same. As a result, $R_{\exp }$ becomes smaller than $R_{\text {calc }}$.

† In 1964, Gaponov and Tyutin [255] suggested studying the neutrinodeuteron interaction involving weak neutral currents. They calculated the cross section of the $v+\mathrm{d} \rightarrow \mathrm{v}+\mathrm{n}+\mathrm{p}$ reaction. 
Reines et al. [186] arrived at the following value for this ratio:

$$
\frac{R_{\text {exp }}}{R_{\text {calc }}}=0.53 \pm 0.20,
$$

which was later corrected by Reines [188] and became $0.69 \pm 0.18$. According to Reines et al. [186], the fact that $R_{\exp } / R_{\text {calc }}$ differs from unity means that oscillations exist in two regions in the $\left(\Delta m^{2}-\sin ^{2} 2 \theta\right)$ plane, which in Fig. 16 are limited by curve 1 .

In 1980 - 1985, Mössbauer and collaborators searched for oscillations of $\tilde{v}_{e}$, first using the research reactor in Grenoble, France [189] (the distance from the reactor to the detector being $R_{1}=8.8 \mathrm{~m}$ ), and then using the reactor in Gösgen, Switzerland $\left(R_{2}=37.9 \mathrm{~m}\right.$ [190], $R_{3}=45.9 \mathrm{~m}$ [191], and $R_{4}=64.7 \mathrm{~m}$ [192]). They measured the spectrum of positrons created in the reaction

$$
v_{\mathrm{e}}+\mathrm{p} \rightarrow \mathrm{n}+\mathrm{e}^{+} .
$$

Events of this type were registered by a device consisting of 30 tanks $(8 \times 20 \times 78 \mathrm{~cm}$ each $)$ filled with a liquid scintillator and connected into five sections, and four wire chambers with ${ }^{3} \mathrm{He}$ placed in between the sections. The overall volume of the scintillator was 3771 .

The search for oscillations consisted in comparing the experimental spectrum of positrons satisfying certain event

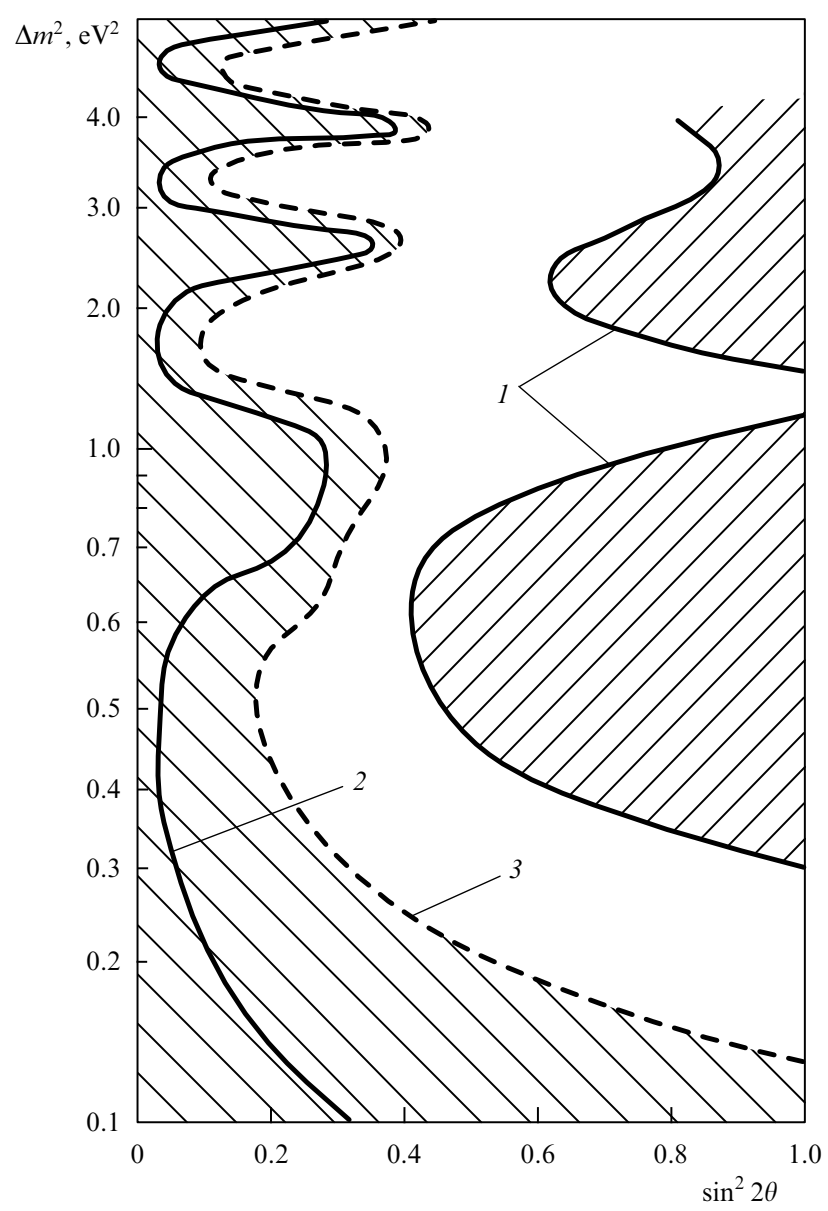

Figure 16. Allowed regions of parameters for neutrino oscillations plotted in the $\Delta m^{2}-\sin ^{2} 2 \theta$ plane: 1 - in the experiment of Reines's group, and 2, 3 - in the experiment of Mössbauer's group (within $68 \%$ and $90 \%$ c.l., respectively). selection criteria in the reaction

$$
\tilde{v}_{\mathrm{e}}+\mathrm{p} \rightarrow \mathrm{n}+\mathrm{e}^{+}
$$

with the calculated positron spectrum, which the detector was supposed to register in the absence of oscillations. The results of such a comparison were formulated as restrictions imposed on the oscillation parameters, which are depicted by curves 2 and 3 in Fig. 16. Comparison of the curves 1,2, and 3 and the results of Afonin et al. [193], which supported Mössbauer's results, suggests that the regions specified by Reines et al. [186] contain no oscillations. Thus the first 'discovery' of reactor antineutrino oscillations proved to be false.

5.7.2 Second 'discovery' of reactor antineutrino oscillations. The dramatic story of the search for reactor antineutrino oscillations had an interesting continuation. The first 'discovery' of oscillations was proved to be false by Mössbauer's group. But in 1984, a subgroup from this body of researchers, who used the Bugey reactor near Lyons, France, announced the discovery of oscillations [194]. The detector and the method that this group used were similar to those in the previous case, and the measurements were made at two distances from the centre of the reactor's core, $R_{1}=13.63 \mathrm{~m}$ and $R_{2}=18.30 \mathrm{~m}$. The results are depicted in Fig. 17 by a

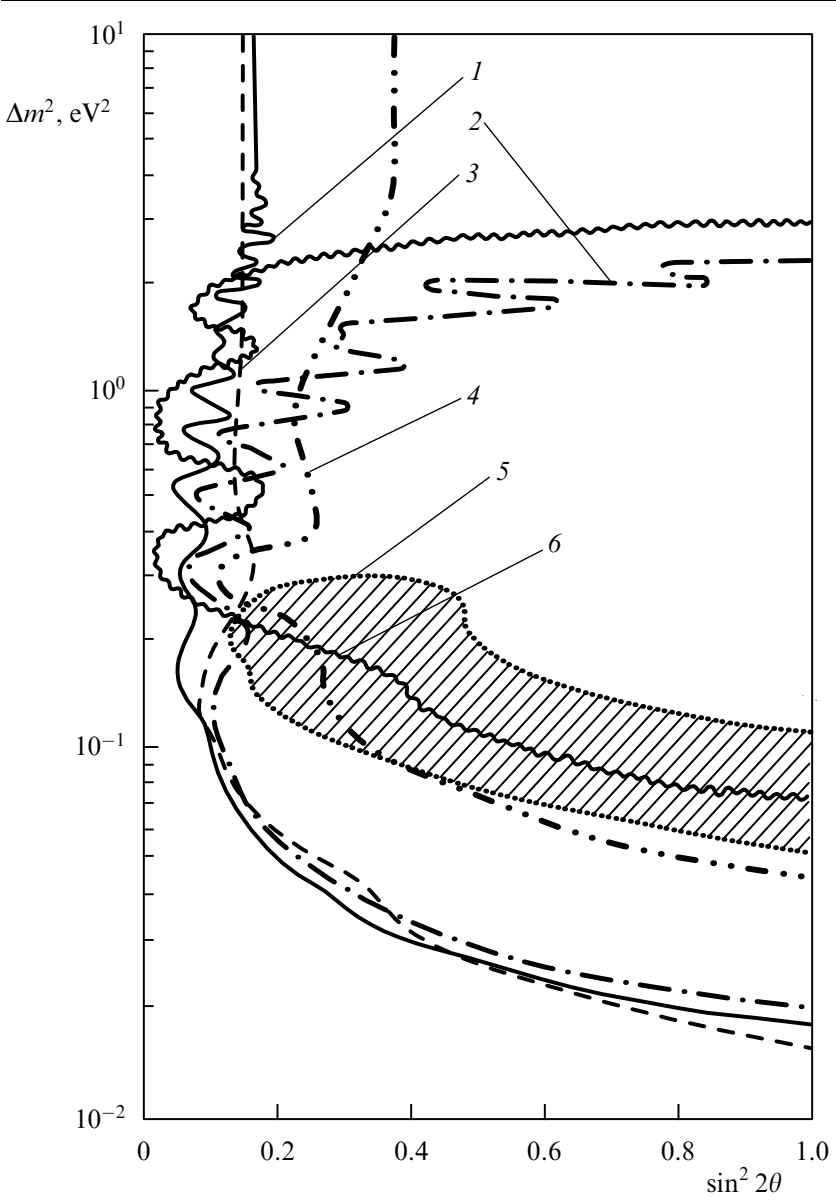

Figure 17. Restrictions on the parameters of oscillations obtained in reactor experiments (the allowed parameter region is to the left and below the curves and inside the hatched band): 1 - Gösgen (absolute result), 2 Gösgen (relative result), 3 - IAE (absolute result), 4 - Rovno (absolute and relative results), 5 - Bugey (relative result), and 6 - Savannah River (relative result). 
hatched section. This was the second time that reactor antineutrino oscillations had been discovered.

The region in which oscillations were discovered, as the researchers (Cavaignac et al. [194]) believed, had noticeably shifted downward along the $\Delta m^{2}$ scale in comparison to Reines's results. Hence a verification of these results was much more difficult. Nevertheless, later several groups of researchers finally proved that this second 'discovery' of $\tilde{v}_{\mathrm{e}}$ oscillations was also false, including the group that 'discovered' the oscillations.

Figure 17 also shows that new results appeared, larger and larger parts of the Bugey region got excluded, so that finally next to nothing was left of it. Here the record value, in the sense of moving down the $\Delta m^{2}$ scale, $0.014 \mathrm{eV}^{2}$, was obtained by researchers of the I V Kurchatov Institute of Atomic Energy (IAE), Russia, who used two reactors in Krasnoyarsk [133].

Vidyakin et al. [133] searched for oscillations by using either the absolute method of comparing the measured interaction cross section of $\tilde{v}_{\mathrm{e}}$ with the calculated cross section or by establishing the ratio of the effects obtained at different distances to the detector ( 32.8 and $92.3 \mathrm{~m}$ ). They used an integral neutrino detector (IND), which made it possible to measure the cross section of inverse beta decay. The detector was a parallelepiped that measured $80 \times 80 \times 97 \mathrm{~cm}$, manufactured from acrylic plastic. It served as a target for $\tilde{v}_{\mathrm{e}}$ and as a moderator for the neutrons generated in the reaction

$$
\tilde{\mathrm{v}}_{\mathrm{e}}+\mathrm{p} \rightarrow \mathrm{n}+\mathrm{e}^{+} .
$$

Each neutron that was slowed down to thermal energies was absorbed by one of the 105 proportional counters with ${ }^{3} \mathrm{He}$. Experiments and calculations showed that the efficiency of the detector amounted to $\varepsilon_{\tilde{v} \mathrm{p}}=31.1 \pm 0.9 \%$.

The detector was protected by a layer of borated polyethylene with a thickness of $30 \mathrm{~cm}$ or more that surrounded the detector on all sides and by a $10-\mathrm{cm}$ layer of electrolytic copper surrounding the detector on four sides. The total amount of substance above the detector corresponded to $600-\mathrm{m}$ water equivalent. In addition, above the detector there was an active shield from cosmic rays that consisted of scintillator plates.

The experiment continued for three years in different modes of operation of the reactors and yielded the following value of the cross section of the $\tilde{v}_{\mathrm{e}} \mathrm{p} \rightarrow \mathrm{ne}^{+}$reaction:

$$
\sigma_{\tilde{v}_{\mathrm{e}} \mathrm{p}}=\left(6.19 \pm 0.2_{\text {stat }} \pm 0.3_{\text {syst }}\right) \times 10^{-43} \frac{\mathrm{cm}^{2}}{\text { fission }} \quad(68 \% \text { c.l. }) .
$$

The following values for the neutrino oscillation parameters were also obtained:

$$
\begin{aligned}
& \Delta m^{2}<0.014 \mathrm{eV}^{2} \text { at } \sin ^{2} 2 \theta=1 \\
& \sin ^{2} 2 \theta<0.14 \text { at } \Delta m^{2} \geqslant 1 \mathrm{eV}^{2} \quad(68 \% \text { c.l. }) .
\end{aligned}
$$

Another group of IAE researchers (Afonin et al. [195]) used the relative measuring method with an ND-1 neutrino detector placed at two distances $(18$ and $25 \mathrm{~m}$ ) from the reactor of the Rovno nuclear power plant in the neutrino laboratory $[196,197]$. The ND-1 detector was a tank containing 2401 (later 10001 ) of a scintillator with a small amount of gadolinium salt dissolved in it. The results of this group are also depicted in Fig. 17.

In addition to the above results, Fig. 17 depicts the preliminary results of Reines's group obtained in experiments that used the Savannah River reactor [198]. The design of the detector was described by Reines in Ref. [188] and earlier in Ref. [199]. The detector's target consists of a central part containing 3001 of a liquid scintillator with an admixture of gadolinium (for detecting neutrons), and a peripheral part containing $1100 \mathrm{l}$ of a scintillator.

5.7.3 Modern reactor experiments. In recent years there have been no sensational discoveries in connection with the search for electron antineutrino oscillations. The results have only amounted to a gradual lowering of the values of $\Delta m^{2}$ checked for absence of oscillations. Today the minimum value of $\Delta m^{2}$ at $\sin ^{2} 2 \theta=1$ has already passed the threshold of $10^{-2} \mathrm{eV}^{2}$ and is moving toward $10^{-3} \mathrm{eV}^{2}$ (see Section 6.6).

Figure 18 depicts the three latest results known to us: that obtained in 1986 in Gösgen [200], obtained in 1994 in Krasnoyarsk [201], and obtained in 1995 at Bugey [185]. The experiments performed in Gösgen were covered in Section 5.7.1. The lower limit of $\Delta m^{2}\left(\right.$ at $\left.\sin ^{2} 2 \theta=1\right)$ that was reached in these experiments is $2 \times 10^{-2} \mathrm{eV}^{2}$.

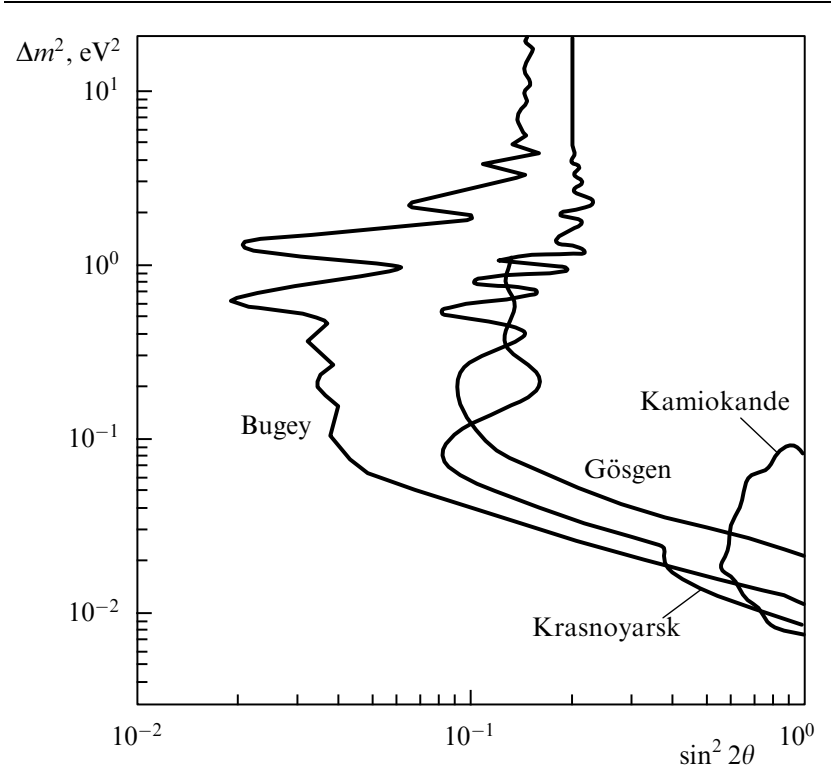

Figure 18. The results of experiments at Bugey, Gösgen, and Krasnoyarsk and a hypothetical range of values for $\left(v_{\mu} \leftrightarrow v_{\mathrm{e}}\right)$-oscillations obtained in the Kamiokande experiment.

A distinctive feature of the Bugey experiment [185] is the use of two reactors separated by a distance of $95 \mathrm{~m}$ and three detectors. This made it possible to measure the antineutrino flux at three distances from the antineutrino source $\left(R_{1}=15 \mathrm{~m}, R_{2}=40 \mathrm{~m}\right.$, and $\left.R_{3}=95 \mathrm{~m}\right)$. The researchers used the ordinary method of comparing the count rates and the positron spectrum obtained in the experiment with the quantities calculated via the Monte Carlo approach. The minimum value of $\Delta m^{2}$ at $\sin ^{2} 2 \theta=1$ reached in this experiment was $10^{-2} \mathrm{eV}^{2}$.

The experiment conducted by the researchers from the Russian Research Centre 'Kurchatov Institute' in the underground laboratory at the Krasnoyarsk Mining Chemical Plant used three reactors separated from the detector by 
$57.0,57.6$, and $231.4 \mathrm{~m}$. The design of the detector is described in Ref. [202]. The detector was a hexagonal prism filled with $458.4 \mathrm{~kg}$ of granulated polyethylene and penetrated by 90 proportional ${ }^{3} \mathrm{He}$-counters with a reduced inherent alphabackground [203]. The efficiency of the detector with respect to neutrons generated in the reaction $\tilde{v}_{\mathrm{e}} \mathrm{p} \rightarrow \mathrm{ne}^{+}$was $\varepsilon=29.4 \pm 1 \%$.

The measurements were carried out over the course of $800 \times 10^{5} \mathrm{~s}$ of on-time for different modes of reactor operations (phased stopping of one reactor, then two reactors, and finally three reactors). As a result of solving an overdetermined system of equations, the cross section of inverse beta decay of the proton was found to be

$$
\sigma_{\tilde{v} \mathrm{p}}=(6.26 \pm 0.26) \times 10^{-43} \frac{\mathrm{cm}^{2}}{\text { fission }} .
$$

The restrictions obtained on the parameters of neutrino oscillations were as follows:

$$
\begin{aligned}
& \Delta m^{2} \leqslant 7.5 \times 10^{-3} \mathrm{eV}^{2} \text { at } \sin ^{2} 2 \theta=1, \\
& \sin ^{2} 2 \theta \leqslant 0.15 \text { for } \Delta m^{2}>5 \times 10^{-2} \mathrm{eV}^{2} .
\end{aligned}
$$

Today these are the most stringent restrictions.

\subsection{Comparison of the results of the search for oscillations} of $v_{\mathrm{e}}, \tilde{v}_{\mathrm{e}}, v_{\mu}$, and $\tilde{v}_{\mu}$ in different experiments

Above we discussed in detail the experiments involved in the search for oscillations of solar electron neutrinos, of atmospheric and accelerator muon neutrinos and antineutrinos, and of reactor electron antineutrinos. Recalling the content of the corresponding sections, we conclude that only in the case of reactor antineutrinos it can be claimed with all certainty that down to the record value of $\Delta m^{2}=7.5 \times 10^{-3} \mathrm{eV}^{2}$ (at $\left.\sin ^{2} 2 \theta=1\right)$ ) obtained in 1994 in Krasnoyarsk no oscillations have been discovered. In all other cases we cannot be certain whether such oscillations exist. Let us discuss these cases separately, starting with solar neutrinos.

The reader will recall that the assumption about the existence of solar neutrino oscillations was made in connection with the discovery of the solar neutrino deficit in Davis's experiments. These results were later corroborated in four independent experiments. But although the phenomenon of the solar neutrino experiment has been established without doubt, there has yet to appear a unique explanation: the loss of $v_{\mathrm{e}}$ can be explained by $\left(v_{\mathrm{e}} \leftrightarrow v_{\mu}\right)$ - or $\left(v_{\mathrm{e}} \leftrightarrow v_{\tau}\right)$-oscillations (in the vacuum of outer space or in the solar depths) or by transformations of the type $v_{\mathrm{e}}^{L} \rightarrow v_{\mathrm{e}}^{R}$ (if the neutrino has a magnetic moment) or, finally, by the limitations of the Standard Solar Model or by the fact that three of the four experiments produced erroneous results.

In the case of atmospheric neutrinos the experiments themselves are not convincing. The values of the $v_{\mu}+\tilde{v}_{\mu}$ to $v_{\mathrm{e}}+\tilde{v}_{\mathrm{e}}$ flux ratios yielded by different experiments either coincide with the calculated values or are smaller by a factor of almost two.

If, however, some future experiments prove that the muon neutrino and antineutrino fluxes decrease, this will indicate that oscillations of the type $v_{\mu} \leftrightarrow v_{\mathrm{e}}$ and/or $v_{\mu} \leftrightarrow v_{\tau}$ (but not $v_{\mu} \leftrightarrow \tilde{v}_{\mu}$ ) do exist in the vicinity of $\Delta m^{2} \simeq 10^{-3}-10^{-1} \mathrm{eV}^{2}$; the problem of the existence (or absence) of oscillations of the $v_{\mu} \leftrightarrow v_{\mathrm{e}}$ type will soon be solved in reactor experiments, which study 'reversed' oscillations of the $v_{\mathrm{e}} \leftrightarrow v_{\mu}$ type (see Section 6.6).
Among the accelerator data gathered in short-baseline experiments of special interest are the results of the LSND experiments, which are proof of existence of a certain mode of $\left(v_{\mu} \leftrightarrow v_{\mathrm{e}}\right)$-oscillations with $\Delta m^{2}$ in the interval $10^{-1}-10 \mathrm{eV}^{2}$ and $\sin ^{2} 2 \theta$ in the interval $10^{-3}-10^{-2}$ (an occurrence experiment). These results, however, have not been substantiated by other experiments (but have not been contradicted in reactor experiments involving the parameter $\sin ^{2} 2 \theta$ ).

Long-baseline experiments usually produce accelerator data pertaining to large $\Delta m^{2}$ (up to $1600 \mathrm{eV}^{2}$ ) and extremely small $\sin ^{2} 2 \theta$ (down to 0.002 ). The study of the region of large values of $\Delta m^{2}$ is justified by the fact that the possibility of such large values as $10 \mathrm{eV}$ for the mass of $v_{\tau}$ cannot be excluded.

On the other hand, reactor data make it possible to move into regions of smaller and smaller values of $\Delta m^{2}$, with $\sin ^{2} 2 \theta$ being relatively large.

Two figures conclude this section. Figure 19 depicts a diagram that shows how the data of the various experiments fill the $\left(\Delta m^{2}-\sin ^{2} 2 \theta\right)$ plane. The shaded area shows what remains of the regions in which the Kamiokande-II and IMB experiments predicted the presence of oscillations. Clearly, with respect to $\left(v_{\mu} \leftrightarrow v_{\mathrm{e}}\right)$-oscillations, this area can be investigated by reactor experiments of the near future (see Section 6.6). Figure 20 depicts the results of the main experiments in the form of the ratio of the registered effect $N_{\text {exp }}$ to the calculated effect $N_{\text {calc }}$ (the latter is obtained on the assumption that there are no oscillations). The case $N_{\exp } / N_{\text {calc }}=1$ corresponds to the absence of oscillations, while for $N_{\exp } / N_{\text {calc }}<1$ there is still hope that such oscillations exist.

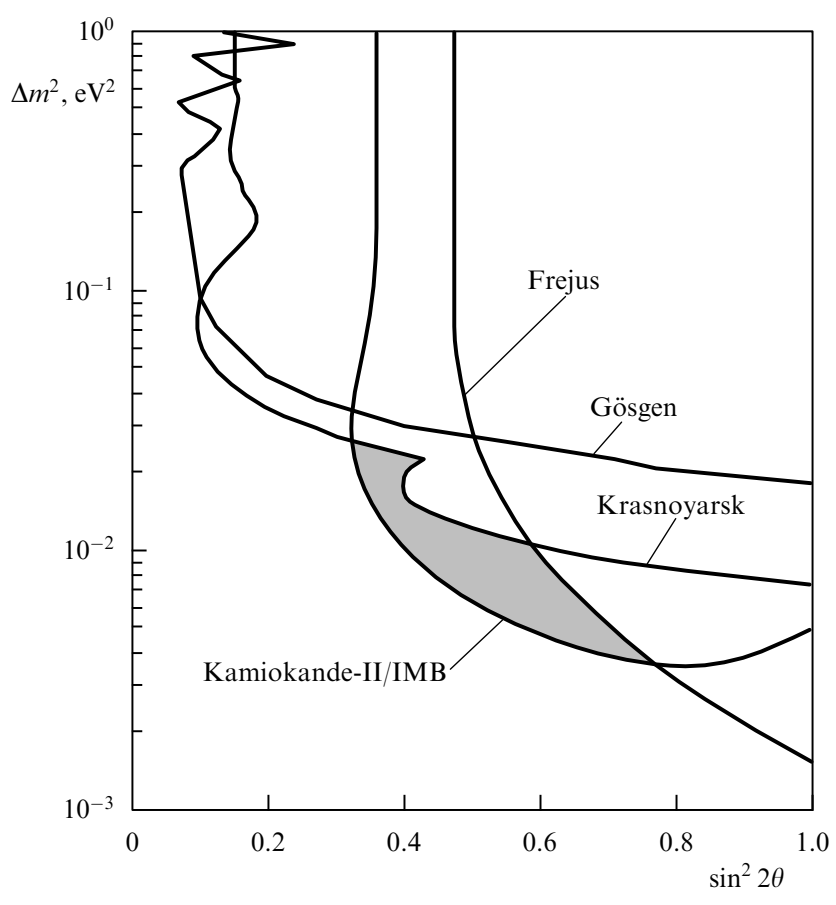

Figure 19. Present limitations on the region of allowed oscillations.

\section{New experimental projects and methods}

We start this section by discussing projects that involve building huge facilities, whose completion will require a lot of time and effort. Then we discuss projects involving very 


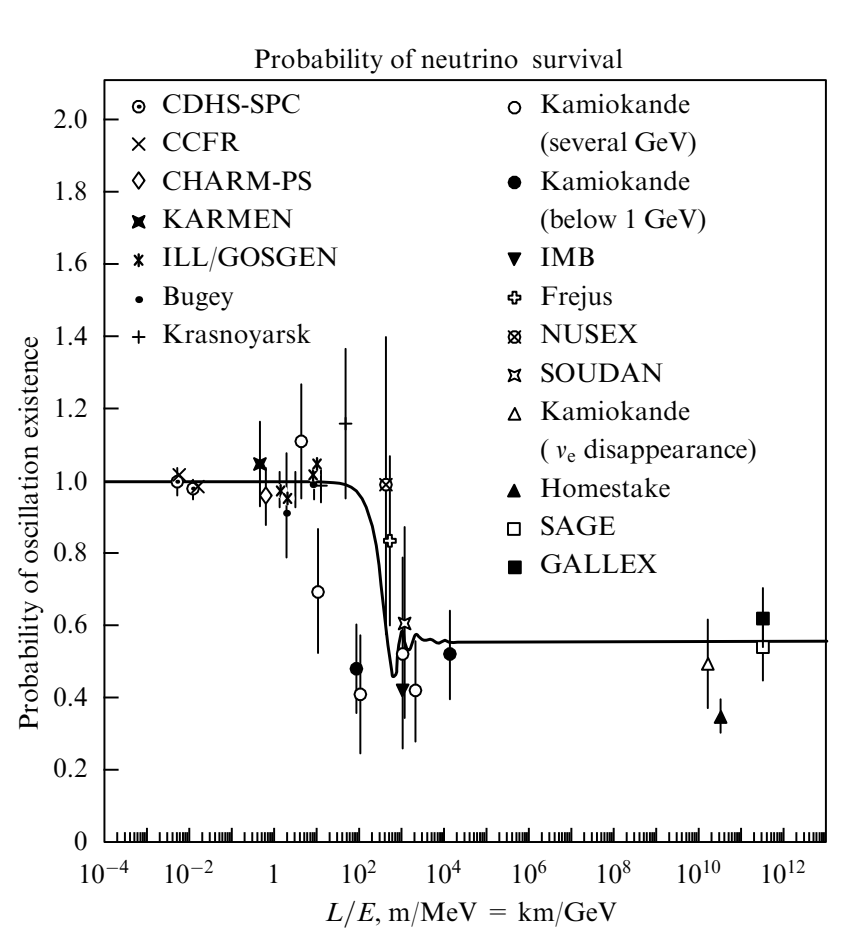

Figure 20. Probability of neutrino 'survival' in oscillations.

large detectors, which are expected to yield important data in the immediate future.

The material of this section is based on the wonderful lecture given by Stanley Wojcicki [9], to whom we would like to express our deep gratitude. There is also a good review in the July, 1996, issue of Physics Today.

\subsection{Very large detectors of $\mu$ and $v$ with targets of unlimited size}

The ever growing demands on the efficiency of neutrino and muon detectors gave birth to the idea of using natural reservoirs filled with water (or ice) as targets [204]. The unlimited sizes of such reservoirs make it possible to design detectors with fantastically large volumes of active substance, of the order of $1 \mathrm{~km}^{3}$. In order to register events, photomultipliers are suspended on cables at a depth of several kilometres 15-20 m apart. The layers and layers of water above the multipliers serve as a good protection against cosmic rays.

Currently there are three projects of this type: DUMAND (Deep Underwater Muon and Neutrino Detection) [205207], NESTOR [208], and 'Băkal' [209]. All have reached the operational prototype stage, with data already being gathered. An innovative detector that uses a huge block of Antarctic ice has been designed by the AMANDA (Antarctic Muon and Neutrino Detector Array) Collaboration [210]. In the Antarctic summer of 1993-1994 near the South Pole, an expedition of researchers drilled canals in the ice (using hot water) to a depth of $800-1000 \mathrm{~m}$ and lowered, by cable, 20 photomultipliers on each cable. A laser light source was used to study the properties of the ice at these depths. It was found that the transparency of the ice is comparable to that of the extremely pure water used in the IMB and Kamiokande detectors, and the number density of air bubbles decreases with increasing depth. Later it was found that at depths greater than $1200 \mathrm{~m}$ there are practically no air bubbles and light propagates without any scattering.
The above superdetectors are chiefly intended for studies in astrophysics and cosmology, but they can also be used to investigate neutrino oscillations (the baseline of CERN's SPS-NESTOR measures $1680 \mathrm{~km}$ ).

\subsection{Superkamiokande}

In Sections 3.2.5 and 5.4.2 we discussed how the large Cherenkov water detector Kamiokande was used to register neutrinos from the burst of SN 1987A and solar neutrinos. In May 1996, the construction of an even larger detector, the Superkamiokande [91], was completed. The reservoir of the target is a large grotto in the Kamioka mine $(250 \mathrm{~km}$ from KEK), filled with $50 \mathrm{kt}$ of water containing the photomultipliers. The transparency of the water has been measured and exposures, in which both solar and atmospheric neutrinos were registered, have been conducted.

It is expected that the new detector will have a lower electron detection threshold $(5 \mathrm{MeV})$ than the Kamiokande device and will make it possible to register up to 8000 solarneutrino events every year. This detector can also be used to study the atmospheric neutrino anomaly, neutrino flares from future supernova bursts, and accelerator neutrino oscillations (see Section 6.5).

\subsection{The underground Sudbury Neutrino Observatory}

The SNO detector [211] sits $2070 \mathrm{~m}$ underground in an active nickel mine in Canada. It is a sphere $12 \mathrm{~m}$ in diameter filled with one kiloton of heavy water and surrounded by seven kilotons of ordinary water. The sphere houses 9500 photomultipliers that are roughly $50 \mathrm{~cm}$ apart.

A distinctive feature of this detector is its capability of registering the full neutrino flux (mainly boron neutrinos), i.e. to detect not only a reaction with charged currents but also a reaction with neutral currents:

$$
\begin{aligned}
& v_{\mathrm{e}}+\mathrm{d} \rightarrow \mathrm{p}+\mathrm{p}+\mathrm{e}^{-}, \\
& v_{\mathrm{x}}+\mathrm{e}^{-} \rightarrow v_{\mathrm{x}}+\mathrm{e}^{-}, \\
& v_{\mathrm{x}}+\mathrm{d} \rightarrow \mathrm{n}+\mathrm{p}+v_{\mathrm{x}}
\end{aligned}
$$

(the neutrons can be detected either by ${ }^{3} \mathrm{He}$-counters or by capture in chlorine, which is introduced into the target in the form of a solution of $\mathrm{MgCl}_{2}$ ).

The possibility of registering reactions with neutral currents is important since calculations of the MikheevSmirnov-Wolfenstein effect suggest that the charged-toneutral current ratio is in the range $0.2-0.6$.

To ensure reliable studies of neutrino flares from future supernova bursts, the SNO detector will be synchronized with the Superkamiokande detector. It will become operational in 1998.

\subsection{Other detectors for solar and atmospheric neutrinos}

Below we list (and briefly describe) some other neutrino detector projects. These detectors will make it possible to refine the energy spectrum of solar neutrinos, which is necessary for a better matching of the various experiments (the reader will recall that to interpret the results of the measurements of the solar neutrino deficit correctly, one must be sure that the spectrum contains no beryllium neutrinos (see Section 5.4.2)).

The Gran Sasso d'Italia laboratory ( $730 \mathrm{~km}$ from SPS) is supposed to house a new detector, IGARUS [212] (of the TPC type described in Section 4.2), filled with liquid argon. The detector will consist of three modules weighing five kilotons 
each and will make it possible to study separately the boronneutrino processes caused by charged and neutral currents.

Two detectors are being developed that will register ppneutrinos. To weaken the effect of Coulomb scattering of lowenergy electrons, the detectors will be filled with helium. One detector, Heron, will register the interaction of neutrinos and superfluid helium [213], and the other, Hellaz, will be of the TPC type with gaseous helium at high pressure [214] or with $12 \mathrm{t}$ of liquid helium [215].

A new detector, called Borexino, is being built at the Gran Sasso d'Italia laboratory to measure the beryllium neutrino flux from the solar spectrum $\left(E_{v}=0.86 \mathrm{MeV}\right)$ [216]. The detector will contain $100 \mathrm{t}$ (300 t in the future) of a liquid ultra-pure scintillator with an extremely weak radioactive background. The photomultipliers and shielding materials will also meet this requirement. The detector will look for elastic scattering processes. The high light yield of the scintillator will guarantee that spectra up to $50 \mathrm{keV}$ will be measured. In addition to beryllium neutrinos, the Borexino detector will be able to measure events with higher energies (involving boron neutrinos).

In 1976, Radhavan [217] proposed an interesting method for registering solar neutrinos. The idea was to detect the products in the following reaction:

$$
v_{\mathrm{e}}+{ }_{49}^{115} \mathrm{In} \rightarrow{ }_{50}^{115} \mathrm{Sn}^{*}+\mathrm{e}^{-} .
$$

There are two appealing features of the method. Firstly, the threshold of reaction (105) is just $119 \mathrm{keV}$, which allows practically all neutrinos generated by solar thermonuclear reactions to be registered. Secondly, as a result of this reaction ${ }_{50}^{115} \mathrm{Sn}^{*}$ is produced in an excited state with a lifetime of roughly $3.3 \mu \mathrm{s}$, and the decay proceeds according to the following scheme:

$$
{ }_{50}^{115} \mathrm{Sn}^{*} \rightarrow{ }_{50}^{115} \mathrm{Sn}+\gamma_{1}(116 \mathrm{keV})+\gamma_{2}(497 \mathrm{keV}),
$$

which makes it possible, due to delayed temporal and geometric coincidences, to suppress the background by several orders of magnitude. Works on fabricating crystal scintillator with an elevated (up to $30-40 \%$ ) content of ${ }_{49}^{115} \mathrm{In}$ are being carried out in Russia by Skorokhvatov's group (Russian Research Centre 'Kurchatov Institute') [218, 219] and in France by Cavaignac's group (ISN, Grenoble). The first encouraging results involving indium-containing crystals doped with Ce were obtained in 1996 by Machulin et al. [220]. A heavy scintillation detector containing indium will make it possible to measure almost the entire solar neutrino spectrum and, in this way, to select the best model of neutrino behaviour from creation to detection.

The reader will recall that in Section 4.3 we already mentioned the NEMO-3 detector in connection with double beta decay studies, and in Section 4.4 we discussed the results planned for a germanium detector that could be obtained in the five years to come.

\subsection{New neutrino experiments involving accelerators}

The most realistic project, which apparently will have been realized by the time this review is published, is the study of the neutrino beam from the $12-\mathrm{GeV}$ KEK synchrotron with the Superkamiokande detector, which sits roughly $250 \mathrm{~km}$ away in the Kamioka mine [221]. The values of $\Delta m^{2}$ that are accessible are in the $10^{-2} \mathrm{eV}^{2}$ range at $\sin ^{2} 2 \theta \simeq 1$.
A more distant Japanese project will involve the neutrino beam from a new $50-\mathrm{GeV}$ proton synchrotron, which will become operational in 2003 [222].

In the US, the MINOS (Main Injector Neutrino Oscillation Search) project is planned to start operation after the year 2000 [223]. The experiment will involve using the Fermilab neutrino beam after modernization, which will amount to building a new main injector. The detector will be sited in the Sudan mine $710 \mathrm{~km}$ away from Fermilab. It will contain roughly $10 \mathrm{kt}$ of active substance with magnetized iron layers (to determine the muon charge) and is designed to register the creation and decay of tau leptons.

In addition to this new detector, the MINOS experiment will use the existing SUDAN-2 detector and the additional detector, which is closer to Fermilab. It is assumed that the MINOS experiment will study not only the parameters of the oscillations but also the oscillation modes.

Similar plans for using neutrino beams from high-energy accelerators are being developed in Europe. Specifically, there is a plan to study the neutrino beam from CERN's SPS in the Gran Sasso d'Italia laboratory, which is $730 \mathrm{~km}$ away, using the above-mentioned IGARUS detector [224], the RICH detector [225], and the NOE (Neutrino Oscillations Experiment) detector [226].

In addition to projects that involve long-baseline experiments, there are short-baseline projects, which will make it possible to study the $\Delta m^{2} \simeq(10-100) \mathrm{eV}^{2}$ range with the smallest possible values of $\sin ^{2} 2 \theta$, which is important if the cosmological assumption that $m_{v_{\tau}} \simeq(1-10) \mathrm{eV}$ is valid.

We know two programs of this kind. One was developed at CERN [227] and the other, at Fermilab [228]. The CERN program will use the CHORUS detector [229], which is a large (roughly one ton) emulsion target for detecting the creation and decay of a tau lepton, and the NOMAD detector [230], which uses thin granulated electronic cameras in a largevolume magnetic field to measure the parameters of taulepton tracks. It is assumed that these detectors will produce results in just a few years.

The Fermilab program relies on the COSMOS experiment [228], which is a part of the MINOS experiment described above. The design of the COSMOS detector is close to that of the CHORUS detector. The distinctive features of this detector are the possibility of fine localization of the peak of the event, a high resolution, and a high precision of tracking. The data from this detector will start to accumulate after the year 2000 .

\subsection{New reactor experiments}

The Kamiokande experiments suggest the possibility of $\left(v_{\mu} \leftrightarrow v_{\mathrm{e}}\right)$ - and/or $\left(v_{\mu} \leftrightarrow v_{\tau}\right)$-oscillations in the $\Delta m^{2} \simeq 10^{-3} \mathrm{eV}^{2}$ range. Hence the importance of studying this range of $\Delta m^{2}$ values in reactor experiments.

In Section 5.6 we saw that modern reactor experiments make it possible to reach values of $\Delta m^{2} \simeq 7.5 \times 10^{-3} \mathrm{eV}^{2}$, and this requires distances of more than $200 \mathrm{~m}$ and detector targets of about $1 \mathrm{t}$. Reducing $\Delta m^{2}$ by a factor of ten requires using distances of about $1 \mathrm{~km}$ and target masses of about $10 \mathrm{t}$. To protect the detector from background radiation it is advisable to place it underground. We know of two projects of this kind: one uses the Palo Verde reactors [231] in the US, and the other the Chooz reactors [232] in France, with the distances to the detectors being $800-1000 \mathrm{~m}$. The detection will use the reaction of inverse beta decay in a hydrogencontaining scintillator with an admixture of gadolinium to 
increase the efficiency of registering neutrons in the delayedcoincidence circuit.

In the Palo Verde experiment the suppression of background noise is achieved by strong segmentation of the 12-ton detector, while in the Chooz experiment a target weighing approximately $5 \mathrm{t}$ 'floats' inside $130 \mathrm{t}$ of a liquid scintillator that acts as an active and passive shield simultaneously.

The rated sensitivity of the experiments involved in the search for neutrino oscillations is estimated to be $\Delta m^{2} \simeq 10^{-3} \mathrm{eV}^{2}$ for $\sin ^{2} 2 \theta=1$ and $\sin ^{2} 2 \theta \approx 0.1$ for large values of $\Delta m^{2}$. This will make it possible to enhance (by a factor of almost ten) the existing limitations on the parameters of $\tilde{v}_{\mathrm{e}} \leftrightarrow \tilde{v}_{\mathrm{x}}$ oscillations (or discover such oscillations in this range of $\Delta m^{2}$ values). In any case, this will solve the problem of the atmospheric neutrino anomaly for the $v_{\mu} \leftrightarrow v_{\mathrm{e}}$ mode. In the fall of 1996, the Chooz experiment produced the first results (still unpublished) of measuring the background noise. They suggest that the rated sensitivity of the future experiments will be attained.

\subsection{A tracer-neutrino beam}

One drawback of all neutrino beams (reactor, solar, atmospheric, and accelerator neutrinos) is that there is an uncertainty in their energy. This leads to a situation in which the results obtained for neutrino beams must be averaged over the neutrino energy spectrum, which is often known inaccurately. More than that, there are certain difficulties in determining the direction of neutrino motion in the beam and the flux value, which makes it extremely difficult to distinguish the effect from the usually strong background noise.

Hence the results we discussed in the previous sections often differ much more strongly than they would if the errors were estimated correctly.

Not so long ago similar difficulties were encountered with photon beams produced by bremsstrahlung, with the spectrum of such beams obeying the $1 / E_{\gamma}$-law. There the problem was solved by placing 'tracers' on the photons. The idea of tracing consists in determining the energy and direction of motion of a bremsstrahlung photon from the energy and direction of motion of the scattered electron that emitted the photon. Naturally, the 'traced' photon (i.e. selected by its energy) must be 'coupled' by a coincidence circuit to the products of the reaction it triggers.

A somewhat similar scheme can be realized in the case of neutrino beams. A team of researchers of the Institute of High-Energy Physics (IHEP, Serpukhov, Russia) developed a method for tracing neutrinos from K-meson decays $\uparrow$ [233, 234]. The idea of neutrino tracing consists in the following. When a reaction triggered by a neutrino is registered, the characteristics of the other particles produced in the decay of a K-meson are measured simultaneously. These measurements are made at the end of the decay base using a special tracing station, which is coupled by a coincidence circuit to the neutrino detector. As a result it becomes possible to establish with high accuracy not only the neutrino's energy but also the point where the neutrino was created and the path to the interaction vertex. The criterion that allows a judgement of whether these calculations are correct is the agreement between the calculated trajectory and the real geometry of the mutual pattern of the different elements of the tracing device (spatial adjustment). The realization of the

$\dagger$ Tracing neutrinos from the $(\pi \rightarrow \mu)$-decay has a number of drawbacks. tracing system will lead to a sharp decrease (by a factor from $10^{2}$ to $10^{3}$ ) in the background signal from untraced neutrinos and other particles.

Denisov et al. [235, 236] describes a project to build a neutrino-tracing station for the accelerator-storage complex (ASC) with a rated energy of $2 \times 3 \mathrm{TeV}$. The energy range for possible kaon creation in such an accelerator is $1-2 \mathrm{TeV}$. The decay of kaons generates neutrinos with an energy of roughly $1 \mathrm{TeV}$. The tracer-neutrino method will make it possible to determine this energy to within several percentage points. At present certain results have been reached in realizing this project.

\subsection{Artificial radioactive isotopes as sources of neutrinos}

In recent years, thanks to the techniques developed at the Russian Research Centre 'Kurchatov Institute' (Moscow), it has become possible to manufacture strong sources of radioactive isotopes, which can be used as sources of neutrinos. The attraction of using such isotopes as ${ }_{26}^{55} \mathrm{Fe}$, ${ }_{24}^{51} \mathrm{Cr}$ [237], ${ }_{61}^{147} \mathrm{Pm}$ [238], ${ }_{38}^{90} \mathrm{Sr}-{ }_{39}^{90} \mathrm{Y}$ [239] and some others as sources of neutrinos lies in the fact that ${ }_{26}^{55} \mathrm{Fe}$ and ${ }_{24}^{51} \mathrm{Cr}$ emit single lines of neutrinos in the process of $\mathrm{K}$-capture with energies of 231 and $756 \mathrm{keV}$, respectively, while the other isotopes produce the well-known neutrino spectra with $E_{v}^{\max }=224 \mathrm{keV} \quad(\mathrm{Pm}) \quad E_{v}^{\max }=546 \quad \mathrm{keV} \quad(\mathrm{Sr})$, and $E_{v}^{\max }=2273 \mathrm{keV}(\mathrm{Y})$ with practically no accompanying gamma quanta. With the real strength of the sources amounting to several megacuries and the lifetimes ranging from 28 days $(\mathrm{Cr})$ to 2.9 years $(\mathrm{Fe})$, such sources can be used in prolonged neutrino experiments. Artificial sources of neutrinos are already being used to calibrate solar neutrino detectors (GALLEX and SAGE). There are also plans to use them in experiments involved in the search for the magnetic moment of the neutrino. Such experiments require sources of neutrinos that have fairly low energies and a well-known spectrum.

\section{Possible practical uses of neutrinos}

\subsection{Neutrino diagnostics of nuclear reactors.}

\section{Estimates of reactor power and composition of core}

When we described the experiments involved in the search for reactor antineutrino oscillations, we considered the reactor as being the only source of neutrinos, without mentioning the processes in which such neutrinos are created. However, the information about these processes is of great practical importance.

It is well known that in a nuclear reactor the thermal energy appears because nuclear fuel 'burns' in a chain nuclear fission reaction. Usually the fuel is ${ }^{235} \mathrm{U}$, which natural uranium contains only in minute quantities (one part in 140 in the relation to the main uranium isotope ${ }^{238} \mathrm{Ut}$. It is also known that in addition to ${ }^{235} \mathrm{U}$ being 'burned' in the reactor, ${ }^{238} \mathrm{U}$ transforms into isotopes of plutonium $\left({ }^{239} \mathrm{Pu}\right.$ and partially ${ }^{241} \mathrm{Pu}$ ), which, like ${ }^{235} \mathrm{U}$, can also serve as a nuclear fuel and facilitate, as the quantity increases, the production of additional energy in the reactor. Finally, not all the Pu burns up in this process - some the it accumulates and, if necessary, can be extracted from the reactor and utilized in other reactors or in nuclear weaponry.

* In thermal-neutron reactors, in addition to natural uranium, the fuel contains uranium enriched in ${ }^{235} \mathrm{U}$. 
This brief description of the processes that take place in a nuclear reactor clearly shows how important it is to control them and, in particular, to monitor the power of the reactor and the build-up of plutonium. The common approach to monitoring reactor power is to use temperature-sensing, neutron-sensing, and other pickup devices. Monitoring the plutonium build-up is a much more complicated problem, which is solved indirectly rather than directly.

However, the two problems can be solved neatly by a direct method that uses the information carried out by the antineutrino flux directly from the reactor's core and transmitted to the detector without any distortions introduced in the flight.

7.1.1 Estimates of reactor power. The solution to the first problem is obvious, at least in principle. The thermal power of the reactor is proportional to the number of fission events per unit time that take place in the reactor. Each event produces beta-active fragments and decay products, which are sources of antineutrinos. Here the average number of antineutrinos emitted in one fission event is known (roughly six). Thus, the antineutrino flux must be proportional to the number of fission events per unit time, i.e. to the thermal power of the reactor. This implies that any detector that registers antineutrinos from the reaction of inverse beta decay may serve as a 'meter' of reactor power.

A detailed study of this problem (and the problem of core composition) was conducted at the neutrino laboratory built under one of the reactors of the Rovno nuclear power plant [240, 241]. From above the laboratory is shielded by layers of special concrete and steel from the gamma quanta and neutrons generated by the reactor and from cosmic rays, and on the sides and from below, as well as from above it is shielded from the natural radioactivity of the soil and rock by a steel casing. The laboratory housed an ND-1 neutrino detector (see Section 5.7.2). Experiments involving this detector corroborated the possibility of actively monitoring the reactor power by measuring the changes in the flux of antineutrinos emitted by the reactor.

7.1.2 Estimates of core composition. The second problem, i.e. estimating the changes in the core in the process of a reactor's operation, proved to be more difficult, since its solution requires not only registering a fairly large number of antineutrinos but also recording the changes in the energy spectrum of these antineutrinos.

The hypothesis of the difference of the spectra of $\tilde{v}_{\mathrm{e}}$ generated by the products of fission of ${ }^{235} \mathrm{U}$ and ${ }^{239} \mathrm{Pu}$ was first proposed by Borovoí and Mikaelyan [240]. A year later Borovoî et al. [242] checked its validity by calculations, and in 1983 Borovor et al. [243] experimentally verified the difference in the spectra of the electrons generated as the fission products of ${ }^{235} \mathrm{U}$ and ${ }^{239} \mathrm{Pu}$, which meant that the spectra of the antineutrinos generated by these nuclides must differ, too. Finally, in 1986, Borovor et al. [244] obtained a direct proof from experiments involving the ND-1 detector that the antineutrino spectra are different.

The experimenters compared the antineutrino spectra measured just before the reactor is shut down (when part of the ${ }^{235} \mathrm{U}$ had burned out and a certain amount of ${ }^{239} \mathrm{Pu}$ had accumulated) and after a new batch of nuclear fuel was loaded (as a result, there was a relatively larger amount of ${ }^{235} \mathrm{U}$ and a smaller amount of ${ }^{239} \mathrm{Pu}$ than before). The results are preliminary and contain considerable errors, but the pattern of the experimental points suggests a change in the antineutrino spectrum (the spectrum from ${ }^{239} \mathrm{Pu}$ is softer).

\subsection{Neutrinos in geophysics.}

\section{Neutrino geology and geodesy}

7.2.1 Using neutrinos in geophysics and estimates of such possibilities. The idea of using neutrinos in geophysics [245248] is based on two features of the neutrino: its fantastically high penetrativity (characterized by a cross section of approximately $0.5 \times 10^{-38} \mathrm{~cm}^{2}$ at $E_{v}=1 \mathrm{GeV}$ ) and the increase in cross section with energy according to a linear lawt.

Indeed, if we assume that the cross section for $1 \mathrm{TeV}$ neutrinos is $\sigma \simeq 0.5 \times 10^{-35} \mathrm{~cm}^{2}$, we easily find that a neutrino's mean free path in, say, silica $\mathrm{SiO}_{2}$, which is the main component of the Earth's minerals, reaches $0.8 \times 10^{11}$ $\mathrm{cm} \simeq 70 D_{\mathrm{E}}$, where $D_{\mathrm{E}}=1.2 \times 10^{9} \mathrm{~cm}$ is the diameter of the Earth.

Thus, only one in seventy $1 \mathrm{TeV}$ neutrinos that travel through the Earth along its diameter will interact with a nucleon, i.e. the neutrino flux entering the detector decreases by roughly $1.5 \%$. If we also allow for the fact that the energy of the neutrinos produced in next-generation accelerators will be higher than $1 \mathrm{TeV}$ and the Earth consists not of silica alone but contains heavier minerals, the effect becomes truly measurable. And since the number of interactions depends on the nucleon number density $n$, i.e. the density of matter, which is different for different minerals, then by changing the direction of the neutrino beam we can, at least in principle, discover deposits of minerals or study the Earth's inner structure.

In the first case, where the neutrino beam is directed along a minor chord, i.e. near the Earth's surface, information about the composition of the Earth's minerals could be obtained by studying the secondary radiation (muonic, acoustic, or radio emissions) registered by detectors that are placed on the surface along the neutrinos' path inside the Earth. In the second case the information is carried by the neutrino beam itself and can be extracted by measuring the beam's intensity as it emerges from the Earth's surface.

It is impossible in this short review to discuss the various aspects of neutrino diagnostics of the Earth in great detail. Below is a list of some problems that are being actively discussed and, possibly, will find a solution in the future.

7.2.2. Neutrino geodesy. The idea of the method consists in making highly precise measurements (to within approximately one centimetre) of the distance between two points on the Earth's surface (or on the ocean bottom) by measuring the time it takes a neutrino to travel this distance. Naturally, the method requires extremely accurate synchronization of the clocks at the two points and a fairly compact (in linear dimensions) blob of neutrinos that leave the source simultaneously. In this sense, the most promising are the linear pion accelerators designed at the Novosibirsk Nuclear Physics Institute [249], in which the acceleration of an unstable particle to a high energy occurs in a single flight through the acceleration gap, with the particle not having enough time to

$\dagger$ Measurements of the total cross sections for $\nu_{\mu}$ and $\tilde{v}_{\mu}$ made in the $1-$ $300 \mathrm{GeV}$ range yielded the following values:

$$
\frac{\sigma_{t}^{(v)}}{E_{\mathrm{v}}}=0.7 \times 10^{-38} \frac{\mathrm{cm}^{2}}{\mathrm{GeV}} ; \quad \frac{\sigma_{t}^{(\tilde{\mathrm{v}})}}{E_{\tilde{\mathrm{v}}}}=0.3 \times 10^{-38} \frac{\mathrm{cm}^{2}}{\mathrm{GeV}} .
$$


decay. Such accelerators should have other useful features (a monochromatic and well-collimated beam of pions and hence neutrinos, relatively high neutrino energies, and high neutrino yields in comparison with ring proton accelerators).

7.2.3. Neutrino geology. It is believed that geological studies could use the above-mentioned secondary radiations generated by a neutrino beam along its path. The first, and apparently the most promising, possibility is related to the study of muon radiation. When the neutrinos pass through homogeneous minerals, the muon flux is at equilibrium. But when the neutrinos travel through a deposit whose atomic composition differs from that of the surrounding minerals, a deviation in the equilibrium muon flux occurs, and this can be registered by a detector at the surface of the Earth. By the nature of the deviation (an increase or decrease in the number of muons) one can estimate the properties of the deposit (heavier or lighter than the surrounding minerals).

Another possibility uses the fact that there arises always an acoustic wave accompanying the propagation of a neutrino beam. This wave forms along the beam's axis as a result of thermal expansion of the medium due to the release of heat by the beam [250, 251]. The idea of the method is that the acoustic signal depends on the properties of the medium and the speed of sound, which can easily be measured if one knows the depth at which the beam travels and the time interval between the moment the beam passes a point at a given depth and the time of arrival of the acoustic signal.

Finally, there is a third possibility, which involves registering long-wave radio emission $\dagger$, similar to Cherenkov radiation, generated by the moving charged particles that accompany the neutrino beam in its travel through the minerals [252, 253]. However, Tsarev and Chechin [248] calculated that the long-wave radio signal emitted by nextgeneration multi-TeV accelerators would have a rather low power.

7.2.4 Global studies of the Earth. Tsarev and Chechin [248] believe that the problems involved in a global study of the Earth, i.e. measurements of the dependence of the density of matter on depth, the study of the Earth's core, and the geodesic measurements mentioned earlier, are very close to being solved, since the energy of the neutrino beams that will be generated by the next generation of accelerators currently under construction is sufficient (see Section 7.2). But even such 'simple' problems require, in addition to building new powerful accelerators, solving the problem of creating such a decay channel whose direction can be changed at will. But this is extremely difficult, since for the neutrinos to have the required energies the length of the decay channel must be $1-10 \mathrm{~km}$ long. Of course, it may be possible to solve this problem by using so-called direct neutrinos, which are generated in the process of direct interaction of protons and the target and in the decay of the resulting extremely shortlived $\left(\tau \simeq 10^{-13} \mathrm{~s}\right)$ charmed particles. Such particles decay before they have time to interact with matter even if a copperbeam-stop absorber is placed in their path (as noted in Section 5.6.2, such an absorber completely stops pions, kaons, and muons).

$\uparrow$ Short-wave radiation is absorbed by soil and rock.

\section{Conclusions}

The gathering of data on neutrino properties was certainly a jerky process: a discovery was usually followed by a long period during which the new phenomenon was studied. Here is a list of dates that were turning points in the development of neutrino physics.

1. December 1930. Pauli predicts the existence of the neutrino and its main properties.

2. 1934. Fermi develops the theory of beta decay (the neutrino and electron do not 'reside' in a nucleus, but are ejected from it at the moment of beta decay).

3. 1936-1942. Leipunsky and Allen find indirect indication of the existence of the neutrino.

4. 1937. Majorana's theory.

5. 1949. Hanna and Pontecorvo provide the first estimate of the neutrino mass.

6. 1953-1956. Reines and Cowan obtain a direct experimental confirmation of the existence of an electron antineutrino.

7. 1955. Davis proves that $\tilde{v}_{\mathrm{e}} \neq v_{\mathrm{e}}$.

8. 1957. The $(\mathrm{V}-\mathrm{A})$-variant of the theory of the weak interaction is developed and the helicity of the neutrino is proved. Pontecorvo hypothesizes about neutrino oscillations.

9. 1962-1963. The $v_{\mu}$ is discovered and it is found that $v_{\mu} \neq v_{\mathrm{e}}$ and $\tilde{v}_{\mu} \neq v_{\mu}$.

10. 1967-1968. The theory of the electroweak interaction is developed by Weinberg, Salam, and Glashow (the Standard Model).

11. 1971. Davis's first experiments in detecting solar neutrinos.

12. 1973. Weak neutral currents are discovered.

13. 1975-1977. The tau lepton is discovered and the existence of tau neutrinos $\left(v_{\tau}\right)$ is predicted (the hypothesis is extremely plausible but is not proved by a direct experiment).

14. 1983. The $\mathrm{W}^{ \pm}$- and $\mathrm{Z}^{0}$-bosons, the quanta of the weak interaction, are discovered.

Now let summarize the results of all the research in this field, especially those obtained in recent years.

1. So far there is not a single experimental fact that contradicts the $(\mathrm{V}-\mathrm{A})$-theory of the weak interaction and the Standard Model of the electroweak interaction.

2. About the neutrino masses $m_{v_{\mathrm{e}}}, m_{v_{\mu}}$, and $m_{v_{\tau}}$ we know the following:

(a) direct measurements of the beta decay of tritium yield $m_{v_{\mathrm{e}}}<4.35 \mathrm{eV}$;

(b) neutrino observations of the burst of SN 1987A yield $m_{v_{\mathrm{e}}}<20 \mathrm{eV}$;

(c) the hot dark matter model yields $m_{v_{\mathrm{e}}} \approx 2 \mathrm{eV}$;

(d) the fact that there is no $2 \beta 0 \mathrm{v}$-decay of ${ }^{76} \mathrm{Ge}$ yields $m_{v_{\mathrm{e}}}^{\mathrm{M}}<0.6 \mathrm{eV}$

(e) from the $(\pi \rightarrow \mu)$-decay it follows that $m_{v_{\mu}}<0.17 \mathrm{MeV}$;

(f) from the tau-lepton decay it follows that $m_{v_{\tau}}<24 \mathrm{MeV}$;

(g) astrophysical estimates yield $m_{v_{\mathrm{e}}}+m_{v_{\mu}}+m_{v_{\tau}}<40 \mathrm{eV}$;

(h) from the see-saw mechanism it follows that $m_{v_{1}} \leqslant 2 \times 10^{-4} \mathrm{eV}, m_{v_{2}} \leqslant 3 \times 10^{-3} \mathrm{eV}$, and $m_{v_{3}} \leqslant 10^{-1} \mathrm{eV}$.

3. The width of the $Z^{0}$-boson decay implies that there can be only three light neutrinos, i.e. right up to $m_{v}=M_{Z^{0} / 2}=45 \mathrm{GeV}$ the only neutrinos that can exist are $v_{\mathrm{e}}, v_{\mu}$, and $v_{\tau}$.

4. The origin of the neutrino mass (the Dirac neutrino mass or the Majorana neutrino mass) has yet to be 
determined, since not a single event that can be associated with $2 \beta 0 v$-decay has been discovered.

5. $2 \beta 2 v$-decay has been discovered in some nuclei, and the measured half-life $T_{1 / 2}(2 \beta 2 v)$ agrees with the predictions of the Standard Model. $2 \beta 0 \mathrm{v}$-decay has not been discovered. The best estimate is $T_{1 / 2}^{0 v}\left({ }^{76} \mathrm{Ge}\right)>6.4 \times 10^{24} \mathrm{y}$.

6 . So far no oscillations of solar, atmospheric, accelerator, or reactor neutrinos have been discovered.

(a) Experiments strongly suggest that there is a deficit of solar neutrinos. However, the explanation of this phenomenon is ambiguous: the reason for the deficit may be vacuum oscillations at $\Delta m^{2} \simeq 10^{-11}-10^{-10} \mathrm{eV}^{2}$ or oscillations in solar matter at $\Delta m^{2} \simeq 10^{-5}-10^{-4} \mathrm{eV}^{2}$ or, finally, the existence of a neutrino magnetic moment $\mu_{v}=10^{-11} \mu_{\mathrm{B}}$. Not a single one of these possibilities has been verified by experiment, which leaves a lot of leeway for research;

(b) the question of whether atmospheric neutrino oscillations occur is still unresolved, since only approximately half of all the experiments suggests that an atmospheric neutrino anomaly actually exists. The possibility that the specific oscillation mode $v_{\mu} \leftrightarrow v_{\tau}$ occurs in the vicinity of $\Delta m^{2} \simeq 10^{-3} \mathrm{eV}^{2}$ needs to be corroborated. In relation to the $v_{\mu} \leftrightarrow v_{\mathrm{e}}$ oscillation mode this problem will soon be solved;

(c) the data on accelerator neutrinos become especially interesting when we consider the LSND detector. The data suggest that the 'emerging' $v_{\mathrm{e}}$ can be interpreted as the result of $\left(v_{\mu} \leftrightarrow v_{\mathrm{e}}\right)$-oscillations in the interval $\Delta m^{2}=10^{-1}-10 \mathrm{eV}^{2}$ and $\sin ^{2} 2 \theta>10^{-3}$. This, however, has yet to corroborated by other experiments (true, the result has not been refuted by reactor experiments on $\left(\tilde{v}_{\mathrm{e}} \rightarrow \tilde{v}_{\mu}\right)$-oscillations; here, however, the $\sin ^{2} 2 \theta \simeq 10^{-3}$ region has not been reached);

(d) in reactor experiments the lower limit of the studied range in the $\left(\Delta m^{2}-\sin ^{2} 2 \theta\right)$ plane is slowly diminishing and has now reached $0.0075 \mathrm{eV}^{2}$ and $10^{-2}-10^{-1}$, respectively. In the near future the attained limits will be $10^{-3} \mathrm{eV}^{2}$ and $10^{-3}$, which will apparently solve the problem of the atmospheric neutrino anomaly for the $\left(v_{\mu} \leftrightarrow v_{\mathrm{e}}\right)$-mode.

Thus, today neutrino physics awaits new discoveries. Along all avenues of research the battle is against background noise; meanwhile, there is slow progress in the direction of smaller values of $m_{v}, \Delta m^{2}$, and $\sin ^{2} 2 \theta$ and larger values of $T_{1 / 2}(2 \beta)$.

So far the lowest recorded bound on the mass of $v_{\mathrm{e}}$ $(4.35 \mathrm{eV})$ was the one obtained in Troitsk in studies of the beta decay spectrum of tritium; the lowest recorded bound on the Majorana neutrino mass $\left(m_{v}<0.6 \mathrm{eV}\right)$ was obtained by the Heidelberg-Moscow Collaboration, and the lowest recorded value of $\Delta m^{2}=0.0075 \mathrm{eV}^{2}$ was obtained by the group working in Krasnoyarsk.

Among the most important discoveries that are expected to be made in the near future are the direct proof of existence of $v_{\tau}$ in an experiment similar to the Reines-Cowan experiment, the solution of the problem of existence (or absence) of oscillations in the atmospheric neutrino anomaly region, and the measurement of $T_{1 / 2}(2 \beta 2 v)$ for several new nuclei (say, ${ }^{136} \mathrm{Xe}$ ) and a further lowering of the values of the neutrino mass obtained through experiments on the beta decay of tritium and in the search for $2 \beta 0 v$-decay. The cherished dream is to discover neutrinoless double beta decay and oscillations, as well as the bursts of new supernovae.

In conclusion we allow ourselves a small lyrical digression. In a verse dedicated to the secrets of the poetical craft, the Russian poetess Anna Akhmatova wrote:
If you but knew out of what rubbish

Poetry grows, quite unabashed,

Like the yellow dandelion by the fence,

Like burdock and goosefoot.

In our craft 'poetry' and 'flowers' do not grow by itself out of 'rubbish.' On the contrary, often the 'rubbish,' or background noise, completely covers the 'flowers,' and it takes years and years to gather a 'bouquet,' a week for a single 'flower' (or event). Undoubtedly, however, the new 'poem' about the secrets of neutrinos will finally be written. The many discoveries and achievements discussed in this review are proof of this.

The authors would like to thank $\mathrm{Yu} \mathrm{V}$ Gaponov, O O Patarakin, and V G Tarasenkov for numerous fruitful discussions and for their help.

\section{References}

1. Pauli W Letter written on December 4, 1930 in Zurich to the participants of a physics meeting held in Tübingen. Reprinted in Collected Scientific Papers by Wolfgang Pauli, Vol. II (Eds R Kronig, V F Weisskopf) (New York: Wiley-Interscience, 1964) p. 1316

2. Wu C S, Mozkowski S A Beta Decay (New York: Wiley-Interscience, 1966) [Translated into Russian (Moscow: Atomizdat, 1970)]

3. Reines F, Cowan C L Phys. Rev. 92830 (1953); Science 124103 (1956)

4. $\quad$ Reines F Rev. Mod. Phys. 68317 (1966)

5. Zatsepin G T, Smirnov A Yu 'Neütrino' in Fizicheskaya Entsiklopediya ('Neutrino', Physical Encyclopedia) Vol. 3 (Moscow: Bol'shaya Rossiǐskaya Entsiklopediya, 1992) p. 258

6. Muto K, Klapdor H V, in Neutrinos (Ed H V Klapdor) (Berlin: Springer, 1988)

7. Gelmini G, Roulet E, Preprint UCLA/94/TEP/36, November 1994, hep-ph-9412278

8. Boehm F, Vogel P Physics of Massive Neutrinos (Cambridge: Cambridge University Press, 1987) [Translated into Russian (Moscow: Mir, 1990)]

9. Wojcicki S Prospects in Neutrino Physics (Stanford: Physics Department of the Stanford University, 1996)

10. Moe M, Vogel P Ann. Rev. Nucl. Part. Sci. 44247 (1994)

11. Moe M K Nucl. Phys. B (Proc. Suppl.) 3836 (1995)

12. Shchepkin M G Usp. Fiz. Nauk 143513 (1984) [Sov. Phys. Usp. 27 413 (1984)]

13. Borovoî A A, Khakimov S Kh Ně̃trinnye Eksperimenty na Yadernykh Reaktorakh (Neutrino Experiments Using Nuclear Reactors) (Moscow: Energoatomizdat, 1990)

14. Weinheimer Ch et al. Phys. Lett. B 300210 (1993)

15. Belesev A I et al. Phys. Lett. B 350263 (1995)

16. Imshennik V S, Nadezhin D K Usp. Fiz. Nauk 156561 (1988) [Sov. Phys. Usp. 31461 (1988)]

17. Pontecorvo B M Usp. Fiz. Nauk 141675 (1983) [Sov. Phys. Usp. 26 575 (1983)]

18. Mukhin K N Eksperimental'naya Yadernaya Fizika Kn. 2: Fizika Elementarnykh Chastits (Experimental Nuclear Physics, Book 2: Physics of Elementary Particles) 5th ed. (Moscow: Energoatomizdat, 1993)

19. Okun' L B Fizika Elementarnykh Chastits (Physics of Elementary Particles) 2nd ed. (Moscow: Nauka, 1988)

20. Rekalo M P Neĭtrino (Neutrino) (Kiev: Naukova Dumka, 1986)

21. 'Review of Particle Properties' Phys. Rev. D 541 (1996)

22. Davis R Phys. Rev. 97766 (1955)

23. Fermi E Zs. Phys. 88161 (1934)

24. Leipunski A I Proc. Cambr. Phil. Soc. 32301 (1936)

25. Allen J S Phys. Rev. 61692 (1942)

26. Lee T D, Yang C N Phys. Rev. 104254 (1956)

27. Wu C S et al. Phys. Rev. 1051413 (1957)

28. Garwin R L, Lederman L M, Wainrich M Phys. Rev. 1051415 (1957)

29. Goldhaber M, Grodzins L, Sunyar A W Phys. Rev. 1091015 (1958) 
30. Landau L Nucl. Phys. 3127 (1957)

31. Salam A Nuovo Cimento 5299 (1957)

32. Lee T D, Yang C N Phys. Rev. 1051671 (1957)

33. Feynman R P, Gell-Mann M Phys. Rev. 109193 (1958)

34. Sudarshan E, Marshak R Phys. Rev. 1091860 (1958)

35. Sakurai J J Nuovo Cimento 7649 (1958)

36. Zel'dovich Ya B, Gershteǐn S S Zh. Eksp. Teor. Fiz. 29698 (1955) [Sov. Phys. JETP 2576 (1955)]

37. Mostovoř Yu A, Mukhin K N, Patarakin O O Usp. Fiz. Nauk 166 987 (1996) [Phys. Usp. 39925 (1996)]

38. Gaponov Yu V, Khafizov R U, in Proc. IV Int. Symp. on Weak and Electromagnetic Interactions in Nuclei: Osaka, Japan, 12-16 June 1995 (Eds H Ejiri, T Kishimito, T Sato) (Singapore: World Scientific, 1995) p. 340

39. Gaponov Yu V, Khafizov R U Yad. Fiz. 591270 (1996) [Phys. At. Nucl. 591213 (1996)]

40. Gaponov Yu V, Khafizov R U Phys. Lett. B 3797 (1996)

41. Glashow S L Nucl. Phys. 22579 (1961)

42. Danby G et al. Phys. Rev. Lett. 936 (1962)

43. Pontecorvo B M Zh. Eksp. Teor. Fiz. 371751 (1959) [Sov. Phys. JETP 101236 (1960)]

44. Danby G et al. Phys. Rev. Lett. 10260 (1963)

45. Gell-Mann M Phys. Lett. 8214 (1964)

46. Zweig G, CERN Report 81821/TH 401 (1964)

47. Christenson J H et al. Phys. Rev. Lett. 13138 (1964)

48. Abov Yu G, Krupchitsky P A, Oratovsky Yu A Phys. Lett. 1225 (1964); Abov Yu G, Krupchitskiı̌ P A Usp. Fiz. Nauk 118 (1) 141 (1976) [Sov. Phys. Usp. 19121 (1976)]

49. Lobashev V M et al. Pis'ma Zh. Eksp. Teor. Fiz. 3268 (1966) [JETP Lett. 3173 (1966)]

50. Weinberg S Phys. Rev. Lett. 191264 (1967)

51. Salam A, in Elementary Particle Theory (Ed. N Svartholm) (Stockholm, 1968)

52. Glashow S L, Illiopoulos J, Maiani L Phys. Rev. D 21285 (1970)

53. Hasert F J et al. Phys. Lett. B 46138 (1973)

54. Aubert J J et al. Phys. Rev. Lett. 33 (23) 1404 (1974)

55. Augustin J-E et al. Phys. Rev. Lett. 33 (23) 1406 (1974)

56. Perl M L, Preprint SLAC-PUB-2153 (1978)

57. Lederman L M Scientific Am. 239 (4) 60 (1978)

58. Arnison G et al. Phys. Lett. B 122 (1) 103 (1983); Phys. Lett. B 126 398 (1983)

59. Banner M et al. Phys. Lett. B 122476 (1983)

60. Rubbia C Rev. Mod. Phys. 57699 (1985)

61. Majorana E Nuovo Cimento 14171 (1937)

62. Weyl H Zs. Phys. 56330 (1929)

63. Akhmedov E Kh, in Fizicheskaya Entsiklopediya (Physical Encyclopedia) Vol. 1 (Moscow: Sovetskaya Entsiklopediya 1988) p. 560

64. Dolgov A D, Zel'dovich Ya B Usp. Fiz. Nauk 130559 (1980) [Sov. Phys. Usp. 23459 (1980)]

65. Weinheimer C, in Proc. IV Int. Symp. on Weak and Electromagnetic Interactions in Nuclei: Osaka, Japan, 12-16 June 1995 (Eds H Ejiri, T Kishimito, T Sato) (Singapore: World Scientific, 1995) p. 232

66. Hanna G C, Pontecorvo B Phys. Rev. 75983 (1949)

67. Langer L M, Moffat R J D Phys. Rev. 88689 (1952)

68. Bergkvist K-E Nucl. Phys. B 39317 (1972)

69. Tretyakov E F et al., in Proc. Neutrino Conf.: Aachen, W. Germany 1976

70. Lubimov V A et al. Phys. Lett. B 94266 (1980); Lubimov V A Zh. Eksp. Teor. Fiz. 81 (4/10) 1158 (1981) [Sov. Phys. JETP 54616 (1981)]

71. Boris S D et al. Pis'ma Zh. Eksp. Teor. Fiz. 45267 (1987) [JETP Lett. 45333 (1987)]

72. Fritschi M et al. Phys. Lett. B 173 (4) 485 (1986)

73. Tret'yakov E F Izv. Akad. Nauk SSSR Ser. Fiz. 39583 (1975)

74. Kawakami H et al. Phys. Lett. B 256105 (1991)

75. Holzschuh E Rep. Prog. Phys. 551035 (1992)

76. Robertson R G H et al. Phys. Rev. Lett. 67 (8) 957 (1991)

77. Robertson R G H, in Particle and Nuclear Astrophysics and Cosmology in the Next Millennium Workshop (Colorado: Snowinass, 1994)

78. Lobashev V M, in Proc. IV Int. Symp. on Weak and Electromagnetic Interactions in Nuclei: Osaka, Japan, 12-16 June 1995 (Eds H Ejiri, T Kishimoto, T Sato) (Singapore: World Scientific, 1995) p. 165
79. Lobashev V M, Spivak P E, Preprint INR P-0291 (Moscow, 1983); Nucl. Instr. Meth. A 240305 (1985)

80. Balashev S et al., in Proc. of WEIN-89 (Montreal, 15-19 May 1989) (Gif-sur-Yvette, France: Frontieres, 1989) p. 295

81. Zatsepin G T Pis'ma Zh. Eksp. Teor. Fiz. 8333 (1968) [JETP Lett. 8 205 (1968)]

82. Morrison D R O Usp. Phys. Nauk 156719 (1988) [Sov. Phys. Usp. 31 619 (1988)]

83. Hirata K et al. Phys. Rev. Lett. 581490 (1987)

84. Bionta R M et al. Phys. Rev. Lett. 581494 (1987)

85. Aglietta M et al. Europhys Lett. 3 1315, 1321 (1987)

86. Dadykin V L et al. Pis'ma Zh. Eksp. Teor. Fiz. 45464 (1987) [JETP Lett. 45593 (1987)]

87. Alekseev E N et al. Pis'ma Zh. Eksp. Teor. Fiz. 45461 (1987) [JETP Lett. 45589 (1987)]

88. Alekseev E N et al. Pis'ma Astron. Zh. 1499 (1988) [Sov. Astron. Lett. 1441 (1988)]

89. Bahcall J N, Glashow S L Nature (London) 326476 (1987)

90. Arnett W, Rosner J Phys. Rev. Lett. 581906 (1987)

91. Totsuka Y, Report University of Tokyo No. ICCR-Report-227-9020 (Tokyo: Tokyo Univ. Publ., 1987)

92. Ewan G T et al., Proposal Report SNO 87-12-1987 issued by Sudbury Neutrino Observatory

93. Albrecht H et al. Phys. Lett. B 291221 (1992)

94. Cinabro D et al. Phys. Rev. Lett. 703700 (1993)

95. Buskulic D et al. Phys. Lett. B 349585 (1995)

96. Goeppert-Mayer M Phys. Rev. 48512 (1935)

97. Majorana E Nuovo Cimento 14171 (1937)

98. Inghram M G, Reynolds J H Phys. Rev. 761265 (1949)

99. Bernatowicz T et al. Phys. Rev. Lett. 692341 (1992)

100. Bernatowicz T et al. Phys. Rev. C 47806 (1993)

101. Kirsten T et al., in Proc. Intern. Conf. Mass Spectroscopy: Kyoto, Japan, 1969 (Tokyo, 1970) p. 501

102. Elliott S R et al. Phys. Rev. C 461535 (1992)

103. Burgess C, Cline J Phys Rev. D 495925 (1994)

104. Pas H et al., in Proc. Int. Workshop on Double Beta Decay and Related Topics: Trento 24.04-5.05.95 (Singapore: World Scientific, 1995)

105. Lou K et al., in Proc. IV Intern. Symposium on Weak and Electromagnetic Interactions in Nuclei: Osaka, Japan, 12-16 June 1995 (Eds H Ejiri, T Kishimito, T Sato) (Singapore: World Scientific, 1995) p. 192

106. Engel J, Vogel P, Zirnbauer M R Phys. Rev. C 37731 (1988)

107. Staudt A, Muto K, Klapdor-Kleingrothaus H V Europhys. Lett. 13 31 (1990)

108. Hubert Ph, in Proc. IV Intern. Symp. on Weak and Electromagnetic Interactions in Nuclei: Osaka, Japan, 12-16 June 1995 (Eds H Ejiri, T Kishimito, T Sato) (Singapore: World Scientific, 1995) p. 184

109. Klapdor-Kleingrothaus H V, in Proc. IV Intern. Symp. on Weak and Electromagnetic Interactions in Nuclei: Osaka, Japan, 12-16 June 1995 (Eds H Ejiri, T Kishimito, T Sato) (Singapore: World Scientific, 1995) p. 174

110. Balysh A et al. Phys. Lett. B 356450 (1995)

111. Klapdor-Kleingrothaus H V Prog. Part. Nucl. Phys. 32261 (1994)

112. Beck M et al. Phys. Rev. Lett. 702853 (1993)

113. 'Heidelberg-Moscow Collaboration' Phys. Lett. B 322176 (1994)

114. 'Heidelberg-Moscow Collaboration' Phys. Lett. B 336141 (1994)

115. Hellmig $\mathbf{J}$ et al., in Proc. Intern. School on Particles and Cosmology (Baksan, Russia, April 19-27 1995)

116. Muto K, Bender E, Klapdor H V Z. Phys. A 334187 (1989)

117. You Ke et al. Phys. Lett. B 26553 (1995)

118. Elliott S R et al. Phys. Rev. C 461535 (1992)

119. Alston-Garnjost M et al. Phys. Rev. Lett. 71831 (1993)

120. Danevich F A et al. Phys. Lett. B 34472 (1995)

121. Alessandrello A et al. Phys. Lett. B 335519 (1994)

122. Vuilleumier J-C et al. Phys. Rev. D 481009 (1993)

123. Moe M K et al. Prog. Part. Nucl. Phys. 32247 (1994); Nucl. Phys. B 3836 (1995)

124. 'NEMO-Collaboration' Nucl. Phys. B (Proc. Suppl.) 35369 (1994)

125. Kume K, in Proc. Int. Workshop on Double Beta Decay and Related Topics: Trento 24.04-5.05.95 (Singapore: World Scientific, 1995)

126. Pontecorvo B M Zh. Eksp. Teor. Fiz. 33549 (1957) [Sov. Phys. JETP 6429 (1958)] 
127. Pontecorvo B M Zh. Eksp. Teor. Fiz. 34247 (1958) [Sov. Phys. JETP 7172 (1958)]

128. Maki Z, Nakagawa M, Sakata S Prog. Theor. Phys. 28 (5) 870 (1962)

129. Pontecorvo B M Zh. Eksp. Teor. Fiz. 531717 (1967) [Sov. Phys. JETP 26984 (1968)]

130. Gribov V, Pontecorvo B Phys. Lett. B 28 (7) 493 (1969)

131. Davis R, Harmer D S, Hoffman K C Phys. Rev. Lett. 201205 (1968)

132. Bilen'kiŭ S M Fiz. Elem. Chastits At. Yadra 18449 (1987) [Sov. J.
[Sart. Part. Nucl. 18188 (1987)]

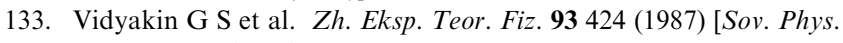
JETP 66243 (1987)]

134. Bahcall J N, Ulrich R K Rev. Mod. Phys. 60297 (1988); Bahcall J N, Pinsonneault M H Rev. Mod. Phys. 64885 (1992); Bahcall J N Nuclear Physics B (Proc. Suppl.) 3898 (1995)

135. Cleveland B Nucl. Phys. B 3854 (1995)

136. Kirsten T, in Proc. IV Intern. Symp. on Weak and Electromagnetic Interactions in Nuclei: Osaka, Japan, 12-16 June 1995 (Eds H Ejiri, T Kishimito, T Sato) (Singapore: World Scientific, 1995) p. 135

137. Anselmann P et al. Phys. Lett. B 327377 (1994)

138. Nico J, in Proc. XXVII Intern. Conf. on High-Energy Physics: Glasgow, UK, July 1994 (Eds P J Bussey, I G Knowles) p. 965

139. Gavrin V A, in Proc. IV Intern. Symp. on Weak and Electromagnetic Interactions in Nuclei: Osaka, Japan, 12-16 June 1995 (Eds H Ejiri, T Kishimito, T Sato) (Singapore: World Scientific, 1995) p. 242

140. Hirata K S et al. Phys. Rev. D 442241 (1991)

141. Krastev P, Petcov S T Phys. Rev. Lett. 721960 (1994)

142. Wolfenstein L Phys. Rev. D 172369 (1978)

143. Wolfenstein L Phys. Rev. D 202634 (1979)

144. Mikheev S P, Smirnov A Yu Usp. Fiz. Nauk 153 (1) 3 (1987) [Sov. Phys. Usp. 30759 (1987)]

145. Mikheyev S P, Smirnov A Yu Talk given at 10th Intern. Conf. on Week Interaction (Savonlinna, Finland, 1985)

146. Mikheev S P, Smirnov A Yu Yad. Fiz. 421441 (1985) [Sov. J. Nucl. Phys. 42913 (1985)]

147. Mikheev S P, Smirnov A Yu Nuovo Cimento C 917 (1986)

148. Mikheev S P, Smirnov A Yu Zh. Eksp. Teor. Fiz. 917 (1986) [Sov. Phys. JETP 644 (1986)]

149. Voloshin M B, Vysotskiĭ M I, Okun' L B Zh. Eksp. Teor. Fiz. 91754 (1986) [Sov. Phys. JETP 64446 (1986)]

150. Veselov A I, Vysotskiı̌ M I, Yurov V P Yad. Fiz. 451392 (1986) [Sov. J. Nucl. Phys. 45865 (1986)]

151. Oakley D et al. Astrophys. J. 43763 (1994)

152. Vogel P, Engel J Phys. Rev. D 393378 (1989)

153. Schreckenbach K et al. Phys. Lett. B 160325 (1985)

154. Hahn A et al. Phys. Lett. B 218365 (1989)

155. Vogel P et al. Phys. Rev. C 241543 (1981)

156. Aleksankin V G et al. Beta- $i$ Antiněttrinnoe Izluchenie Yader (Beta and Antineutrino Emission by Nuclei) (Ed P M Rubtsov) (Moscow: Energoatomizdat, 1989)

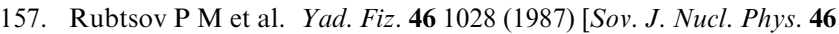
591 (1987)]

158. Bakalyarov A M, Kopě̌kin V I, Mikaelyan L A, Preprint IAE-5973/ 2 (Moscow: Inst. At. En., 1996)

159. Koperkin V, Mikaelian L, Sinev V, Preprint IAE-5980/2 (Moscow: Inst. At. En., 1996)

160. Reines F, Gurr H S, Sobel H W Phys. Rev. Lett. 37315 (1976)

161. Vidyakin G S et al. Pis'ma Zh. Eksp. Teor. Fiz. 55212 (1992) [JETP Lett. 55206 (1992)]

162. Derbin A V et al. Pis'ma Zh. Eksp. Teor. Fiz 57755 (1993) [JETP Lett. 58768 (1993)]

163. Derbin A V Yad. Fiz. 57236 (1994) [Phys. At. Nucl. 57222 (1994)]

164. Domogatskiĭ G V, Nadezhin D K Yad. Fiz. 121233 (1970) [Sov. J.
[S Nucl. Phys.]

165. Akhmedov E Kh, Khlopov M Yu, Preprint M V Keldysh Institute of Applied Mathematics, USSR Acad. Sci., No. 89 (Moscow, 1987)

166. Akhmedov E Kh, Bychuk O V Zh. Eksp. Teor. Fiz. 95444 (1989) [Sov. Phys. JETP 68250 (1989)]

167. Akhmedov E Kh, Lanza A, Petcov S T hep-ph-9411299 FTUV/94/ 64 IFIC/94/67, SISSA 169/94/A-EP

168. Hirata K S et al. Phys. Lett. B 280146 (1992)

169. Fukuda Y et al. Phys. Lett. B 335237 (1994)

170. Boliev M M et al. Yad. Fiz. 341418 (1981) [Sov. J. Nucl. Phys.]
171. Becker-Scendy R et al. Phys. Rev. D 463720 (1992)

172. Allison W et al., ANL HEP CP-93-34; Goodman M Nucl. Phys. B 38 220 (1994); Kafka T, Talk at the TAUP' 93 Conf. (Gran Sasso, Sept. 1993)

173. Berger Ch et al. Phys. Lett. B 227489 (1989)

174. Aglietta M et al. Europhys. Lett. 8611 (1989)

175. Dydak F et al. Phys. Lett. B 134281 (1984)

176. Bergsma F et al. Phys. Lett. B 142103 (1984)

177. Stockdale I E et al. Phys. Rev. Lett. 521384 (1984)

178. Ahrens L A et al. Phys. Rev. D 312732 (1985)

179. Athunassopoulos C et al. Phys. Rev. Lett. 752650 (1995)

180. Stancu I N, in Proc. IV Intern. Symp. on Weak and Electromagnetic Interactions in Nuclei: Osaka, Japan, 12-16 June 1995 (Eds H Ejiri, T Kishimito, T Sato) (Singapore: World Scientific, 1995) p. 156

181. Drexlin G Prog. Part. Nucl. Phys. 32375 (1994)

182. Kleinfeller J, in Proc. IV Intern. Symp. on Weak and Electromagnetic Interactions in Nuclei: Osaka, Japan, 12-16 June 1995 (Eds H Ejiri, T Kishimito, T Sato) (Singapore: World Scientific, 1995) p. 591

183. Hill J E Phys. Rev. Lett. 752654 (1995)

184. Borodovsky L et al. Phys. Rev. Lett. 68274 (1992)

185. Achkar B et al. Nucl. Phys. B 434503 (1995)

186. Reines F, Sobel H W, Pasierb E Phys. Rev. Lett. 45 (16) 1307 (1980)

187. Pasierb E et al. Phys. Rev. Lett. 43 (2) 96 (1979)

188. Reines F Nucl. Phys. A 396469 (1983)

189. Boehm F et al. Phys. Lett. B 97 (2) 310 (1980)

190. Vuilleumier J L et al. Phys. Lett. B 114 (4) 298 (1982)

191. Gabathuler K G et al. Phys. Lett. B 138449 (1984)

192. Zacek V et al. Phys. Lett. B 164193 (1985)

193. Afonin A I et al. Pis'ma Zh. Eksp. Teor. Fiz. 38361 (1983) [JETP Lett. 38436 (1983)]

194. Cavaignac J F et al. Phys. Lett. B 148387 (1984)

195. Afonin A I et al. Pis'ma Zh. Eksp. Teor. Fiz. 44111 (1986) [JETP Lett. 44142 (1986)]

196. Borovoĭ A A, Mikaelyan L A At. Energ. 54 (2) 143 (1983) [Sov. J. At. Energy (1983)]

197. Borovoĭ A A et al., Preprint IAE-4245/14 (Moscow: Inst. At. En., 1986)

198. Boehm F, in Proc. Intern. Symp.: Osaka, Japan, June 1986 (Osaka: University Publ.) p. 439

199. Reines F Usp. Fiz. Nauk 77287 (1962)

200. Zacek V et al. Phys. Rev. D 342621 (1986)

201. Vidyakin G S et al. Pis'ma Zh. Eksp. Teor. Fiz. 59390 (1994) [JETP Lett. 59364 (1994)]

202. Vidyakin G S et al. Zh. Eksp. Teor. Fiz. 98764 (1990) [Sov. Phys. JETP 71424 (1990)]

203. Vidyakin G S et al. Prib. Tekh. Eksp. 32 (4) 70 (1989) [Instrum. Exp. Tech. 32809 (1989)]

204. Markov M A, in Proc. of 1960 Annual Intern. Conf. on High-Energy Physics (Rochester, 1960) p. 578

205. Berezinskiĭ V S, Zatsepin G T Usp. Fiz. Nauk 1223 (1977) [Sov. Phys. Usp. 203 (1977)]

206. Learned J G, Eichler D Sci. Amer. 244 (2) 104 (1981)

207. Stanger B J Nucl. Phys. B 14A 153 (1990)

208. Katsanowas S, in Workshop on Weak Interactions and Neutrinos (Talloires, France, September 1995)

209. Belolaptikov L A et al. Nucl. Phys. B 19388 (1991)

210. Askebjer P et al. Nucl. Phys. B 38283 (1995)

211. Aardsma G et al. Phys. Lett. B 194321 (1987)

212. Revol J P, Preprint CERN PPE/93-01 (1993); Rubbia C, Report CERN PPE/93-08 (1993)

213. Lanou R E, Maris H J, Seidel G M Phys. Rev. Lett. 582498 (1987)

214. Arzarello F et al., Preprint LPC 94-28 (Paris, 1994)

215. Ypsilantis T et al., Preprint LPC 92-31 (1992); Bonvicini G Nucl. Phys. B 35441 (1992)

216. Arpesella C et al. Nucl. Phys. B 28A 486 (1992)

217. Radhavan R S Phys. Lett. 37254 (1976)

218. Bakalyarov A M et al., Preprint IAE-5921/5 (Moscow: Inst. At. En., 1995)

219. Bakalyarov A M et al., Preprint IAE-5942/2 (Moscow: Inst. At. En., 1995)

220. Machulin I N et al., Preprint IAE-6016/2 (Moscow: Inst. At. En., 1996)

221. Nishikawa K, INS Report-924 (April 1992) 
222. Nishikawa $\mathrm{K}$, in Workshop on Weak Interactions and Neutrinos (Talloires, France, September 1995)

223. Able E et al., MINOS Collaboration P-875 (Fermilab, February 1995)

224. 'The IGARUS Collaboration' LNGS-24/99 II (May 1994)

225. Ypsilantis T et al., Preprint LPC-96-01 (January 1996)

226. Barbarino G C et al., NOE: atmospheric and long baseline neutrino oscillations experiment (December 1995)

227. Winter K Nucl. Phys. B 38211 (1995)

228. Schneps J Nucl. Phys. B 38220 (1995)

229. De Jong M et al., CERN PRE/93-131 (July 20, 1993)

230. Di Lella L Nucl. Phys. B 31319 (1993)

231. Piepke A, in Proc. IV Intern. Symp. on Weak and Electromagnetic Interactions in Nuclei: Osaka, Japan, 12-16 June 1995 (Eds H Ejiri, T Kishimito, T Sato) (Singapore: World Scientific, 1995) p. 599

232. De Kerret $\mathrm{H}$, in Workshop on Weak Interactions and Neutrinos (Talloires, France, September 1995)

233. Boikov A A et al., Preprint IHEP 80-156 (Protvino, USSR, 1980)

234. Boikov A A et al., Preprint IHEP 80-158 (Protvino, USSR, 1980)

235. Denisov S P et al., Preprint IHEP 81-98 (Protvino: IHEP Publ., 1980)

236. Denisov S P et al., in Materialy Rabochego Soveshchaniya: Fizicheskie Issledovaniya na Uskoritel'no-Nakopitel'nom Komplekse IFVE (Proc. Workshop on Physical Research Using the IHEP Accelerator-Storage Complex) (Serpukhov: IHEP Publ., 1982) p. 3, p. 167

237. Griber M et al., Preprint DAPNIA-SPP-96-06 (1996)

238. Barabanov I R et al. Astroparticle Physics 5159 (1996)

239. Zaimidoroga O A JINR Rapid Commun. 5 (56) (1992)

240. Borovoĭ A A, Mikaelyan L A, Preprint IAE-2763 (Moscow: Inst. At. En., 1976)

241. Borovoū A A, Mikaelyan L A At. Energ. 44508 (1978) [Sov. J. At. Energy 44589 (1978)]

242. Borovoĭ A A, Dobrynin Yu L, Kopeĭkin V I Yad. Fiz. 25264 (1977) [Sov. J. Nucl. Phys.]

243. Borovoĭ A A, Klimov Yu V, Koperkin V I Yad. Fiz. 371345 (1983) [Sov. J. Nucl. Phys. 37801 (1983)]

244. Bogatov S A et al., Preprint IAE-4284/2 (Moscow: Inst. At. En., 1986)

245. Placci A, Zavattini E, CERN Report (October 1973)

246. Volkova L V, Zatsepin G T Izv. Akad. Nauk SSSR Ser. Fiz. 381060 (1974) [Bull. Acad. Sci. USSR, Phys. Ser.]

247. Tsarev V A Usp. Fiz. Nauk 147426 (1985) [Sov. Phys. Usp. 28940 (1985)]

248. Tsarev V A, Chechin V A Fiz. Elem. Chastits At. Yadra 17 (3) 389 (1986) [Sov. J. Part. Nucl. 17167 (1986)]

249. Balakin V E, Skrinskiı̌ A I Lineinye Vstrechnye Puchki-Perspektivy Razvitiya (Linear Colliding Beams - Outlook of Development) (Moscow: Moscow Engineering Physics Institute, 1984)

250. Askar'yan G A At. Energ. 3162 (1957) [Sov. J. At. Energy]

251. Askar'yan G A, Dolgoshein B A Pis'ma Zh. Eksp. Teor. Fiz. 25232 (1977) [JETP Lett. 25213 (1977)]

252. Askar'yan G A Pis'ma Zh. Eksp. Teor. Fiz. 39334 (1984) [JETP Lett. 39402 (1984)]

253. Tsarev V A, Chechin V A, Preprint P N Lebedev Physics Institute No. 248 (Moscow, 1984)

254. Gaponov Yu V Usp. Fiz. Nauk 102211 (1970) [Sov. Phys. Usp. 13 $511(1971)]$

255. Gaponov Yu V, Tyutin L V Zh. Eksp. Teor. Fiz. 471826 (1964) [Sov. Phys. JETP 201231 (1965)] 